\title{
MINIMAL ENERGY PROBLEMS FOR STRONGLY SINGULAR RIESZ KERNELS
}

\author{
HELMUT HARBRECHT, WOLFGANG L. WENDLAND, AND NATALIA ZORII
}

\begin{abstract}
We study minimal energy problems for strongly singular Riesz kernels $|\mathbf{x}-\mathbf{y}|^{\alpha-n}$, where $n \geq 2$ and $\alpha \in(-1,1)$, considered for compact $(n-1)$-dimensional $C^{\infty}$-manifolds $\Gamma$ immersed into $\mathbb{R}^{n}$. Based on the spatial energy of harmonic double layer potentials, we are motivated to formulate the natural regularization of such minimization problems by switching to Hadamard's partie finie integral operator which defines a strongly elliptic pseudodifferential operator of order $\beta=1-\alpha$ on $\Gamma$. The measures with finite energy are shown to be elements from the Sobolev space $H^{\beta / 2}(\Gamma), 0<\beta<2$, and the corresponding minimal energy problem admits a unique solution. We relate our continuous approach also to the discrete one, which has been worked out earlier by D.P. Hardin and E.B. Saff.
\end{abstract}

\section{INTRODUCTION}

The classical Gauss problem of minimizing the Coulomb energy to solve the problem of Thomson and its generalization to Riesz potentials together with the discretization is the basic problem of many applications (in [3] are listed coding theory, cubature formulas, tight frames and packing problems). In the works [2], [3], [6], [10], and [11], the discretization is obtained by approximating the minimizing charges by a distribution of finitely many Dirac measures on the given manifold.

If the number of Dirac points tends to infinity, then the minimizing densities approach distributions in the form of Sobolev space elements. Therefore, in [8], [9], [18], the minimizing measures are considered as distributions in Hilbert spaces of finite Riesz energy. This continuous setting is simpler and more efficient from the numerical point compared to the discrete approach in [2], [3], [6], [10].

For potentials with Riesz kernel $|\mathbf{x}-\mathbf{y}|^{\alpha-n}$, where $1<\alpha<n$, and Borel measures supported on a given $(n-1)$-dimensional manifold $\Gamma$ immersed into $\mathbb{R}^{n}$, a surface potential is generated, which on $\Gamma$ defines a boundary integral operator with weakly singular kernel. This boundary integral operator is a pseudodifferential operator of negative order $\beta=1-\alpha$ if $\Gamma \in C^{\infty}$. The energy space of this pseudodifferential operator on $\Gamma$ is thus the Sobolev space $H^{\beta / 2}(\Gamma)$ of distributions and the minimizing measure of finite energy is an element of this Sobolev space. Hence, the determination of the minimizer is reduced to an optimization problem with a quadratic functional which is defined in terms of the single layer Riesz potential on $\Gamma$. The strong ellipticity of the corresponding pseudodifferential operator in $\mathbb{R}^{n}$ and its trace on $\Gamma$ then provides the coerciveness of the associated quadratic functional. For $\alpha=2$, which corresponds to the Newtonian kernel, the Riesz energy of the single layer potential is just its Dirichlet integral over $\mathbb{R}^{n} \backslash \Gamma$.

In this paper, however, we consider the Riesz kernels with $\alpha \in(-1,1)$. For $\alpha=0$, in classical potential theory, the energy of the harmonic double layer potential in $\mathbb{R}^{n} \backslash \Gamma$ now equals the Riesz energy if we define the latter as to be Hadamard's partie finie integral of the hypersingular potential - which is the natural distributional regularization (see Section 2 where $\Gamma$ is a $(n-1)$-dimensional planar bounded domain in $\mathbb{R}^{n}$ ).

Let $\Gamma=\bigcup_{i \in I} \Gamma_{i}$ where $\Gamma_{i}, i \in I$, are finitely many compact, connected $(n-1)$-dimensional $C^{\infty}$-manifolds immersed into $\mathbb{R}^{n}$. In Section 3, we then consider the Riesz potential as a pseudodifferential operator just on $\Gamma$ since we cannot use its extension to $\mathbb{R}^{n}$ (for $\alpha \neq 0$, the transmission conditions [12, Theorem 8.3.11] are not satisfied). We call the bilinear form with the strongly singular partie finie integral of the Riesz kernel the energy of the Riesz potential. The partie finie

1991 Mathematics Subject Classification. 31B10, 31C15, 49J35, 45L10.

Key words and phrases. Minimal energy problem; strongly singular Riesz kernel; pseudodifferential operators. 


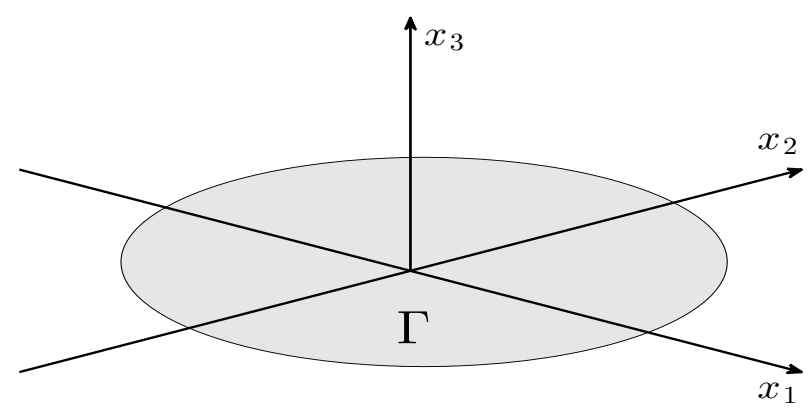

FiguRE 1. Illustration of the geometrical setting.

integral operator with the hypersingular Riesz kernel defines now a strongly elliptic pseudodifferential operator $V_{\beta}$ of positive order $\beta=1-\alpha$ on $\Gamma$.

In contrast to the analysis of weakly singular Riesz kernels provided earlier by the authors in [8], [9], in the case under consideration, the trace theorem in $H^{-\beta / 2}(\Gamma)=V_{\beta} H^{\beta / 2}(\Gamma)$ is not valid anymore, because of the negativity of the order $-\beta / 2$, cf. [1]. Nevertheless, we have succeeded in overcoming this difficulty, and we have shown that all the Borel measures on $\Gamma$ with finite Riesz energy whose restriction on any $\Gamma_{i}$ takes sign either +1 or -1 form a certain cone in the Sobolev space $H^{\beta / 2}(\Gamma), 0<\beta<2$. This is our main result in Section 3, Theorems 3.3 and 3.4. In this framework, the corresponding Gauss variational problem admits a unique solution which belongs to $H^{\beta / 2}(\Gamma)$, which is a compact subspace of $L_{2}(\Gamma)$. These results have again a potential theoretic meaning in the particular situation $\alpha=0$ in relation to the harmonic double layer potential as explained in Section 5.

In the fundamental work [11] by D.P. Hardin and E.B. Saff, discrete minimal energy problems have been investigated. There, the discrete Riesz energies are obtained by distributing a finite number $(N)$ of evenly weighted Dirac measures on a compact $(n-1)$-dimensional manifold $A$ where the set $\mathbf{x}=\mathbf{y}$ is excluded. Then, the discrete minimal Riesz energy determines an optimal geometric arrangement of the $N$ distinct Dirac points on $A$. In [11], three cases are distinguished: (i) the Riesz kernel is weakly singular, (ii) the case $\alpha=1$ (see [14]), and (iii) the hypersingular case $\alpha<1$. For all these three cases, the behavior of the discrete minimal energies for $N$ tending to infinity is explicitly determined (see Section 6 below for details). In the works [2], [3], and [4], these results are generalized to more general Riesz kernels with weights.

During a miniworkshop in August 2012 in Stuttgart with E.B. Saff, D.P. Hardin, and P.D. Dragnev, we have learned from them that in the hypersingular case the discretized minimal energies tend to infinity if the number of Dirac basic points approaches infinity and at the same time those minimizing charges tend to a charge with a constant density. This discussion inspired us to pick up this topic gratefully in our paper and to analyze also this approach by cutting out the set $|\mathbf{x}-\mathbf{y}| \leq \delta$ of $\Gamma \times \Gamma$ where $\delta>0$. We first figure out the idea in Section 6 by studying a perturbed Riesz energy problem. Then, in Section 7, we perform the computations in detail for the punched Riesz energy problem and give an asymptotic expansion of the solution in the corresponding family of finite energy spaces for $\delta \rightarrow 0$. In particular cases (see Corollary 7.5 for details), the minimizers tend to a constant distribution on $\Gamma$ while the corresponding minimal energies tend to infinity.

\section{Motivation. The energy of the Laplacian's double layer potential}

We shall motivate our approach by an example from potential theory where $\alpha=0$, i.e. $\beta=1$. To this end, let $\Gamma \subset \mathbb{R}^{n-1}$ be a planar bounded domain in $\mathbb{R}^{n}$ and $\mathbf{x}=\left(\mathbf{x}^{\prime}, x_{n}\right) \in \mathbb{R}^{n}$ with $\mathbf{x} \in \Gamma$ when $x_{n}=0$, see Figure 1 for an illustration. 
The double layer potential of the Laplacian with given dipole charge density $\varphi\left(\mathbf{y}^{\prime}\right)$ is given by

$$
\begin{aligned}
& U_{\boldsymbol{\varphi}}(\mathbf{x}):=-(\mathbf{W} \boldsymbol{\varphi})(\mathbf{x}):=-\int_{y_{n}=0} \boldsymbol{\varphi}\left(\mathbf{y}^{\prime}\right) \frac{\partial E}{\partial y_{n}}\left(\left(\mathbf{y}^{\prime}, 0\right), \mathbf{x}\right) \mathrm{d} \mathbf{y}^{\prime} \\
&=-c_{n} \int_{\mathbf{y}^{\prime} \in \Gamma} \frac{\left(\mathbf{x}-\left(\mathbf{y}^{\prime}, 0\right)\right) \cdot \mathbf{e}_{n}}{\left|\mathbf{x}-\left(\mathbf{y}^{\prime}, 0\right)\right|^{n}} \boldsymbol{\varphi}\left(\mathbf{y}^{\prime}\right) \mathrm{d} \mathbf{y}^{\prime}
\end{aligned}
$$

for $\mathbf{x} \in \mathbb{R}^{n}$ with $x_{n} \neq 0$ and $c_{n}=\frac{1}{\omega_{n}}=(2(n-1) \pi)^{-1}$. The fundamental solution for the Laplacian is given by

$$
E(\mathbf{x}, \mathbf{y})=c_{n}|\mathbf{x}-\mathbf{y}|^{2-n} .
$$

The vector $\mathbf{e}_{n}=(0, \ldots, 1)^{\top}$ is the $n$-th basis vector of $\mathbb{R}^{n}$ and the unit normal vector on $\Gamma$. If $\varphi$ is continuous at $\mathbf{x}^{\prime}$, then there holds the jump relation

$$
\begin{aligned}
\mathbb{R}_{ \pm}^{n} \ni \mathbf{x} & \rightarrow\left(\mathbf{x}^{\prime}, 0\right): \\
U_{\varphi}(\mathbf{x}) & \rightarrow \mp \frac{1}{2} \varphi\left(\mathbf{x}^{\prime}\right)-\int_{\mathbf{y}^{\prime} \in \Gamma \backslash\left\{\mathbf{x}^{\prime}\right\}} \frac{\left(\left(\mathbf{x}-\left(\mathbf{y}^{\prime}, 0\right)\right) \cdot \mathbf{e}_{n}\right.}{\left|\mathbf{x}-\left(\mathbf{y}^{\prime}, 0\right)\right|^{n}} \boldsymbol{\varphi}\left(\mathbf{y}^{\prime}\right) \mathrm{d} \mathbf{y}^{\prime}=\mp \frac{1}{2} \varphi\left(\mathbf{x}^{\prime}\right)-0
\end{aligned}
$$

since for $\mathbf{x} \in \Gamma$ and $\mathbf{y} \in \Gamma \backslash\{\mathbf{x}\}$ the scalar product $(\mathbf{x}-\mathbf{y}) \cdot \mathbf{e}_{n}=0$ and, hence, the integral vanishes. Consequently, the harmonic potential $U_{\boldsymbol{\varphi}}(\mathbf{x})$ solves the transmission problem in $\mathbb{R}^{n} \backslash \Gamma$

$$
[U]_{\Gamma}:=U_{\varphi}\left(\mathbf{x}^{\prime},-0\right)-U_{\varphi}\left(\mathbf{x}^{\prime},+0\right)=\varphi\left(\mathbf{x}^{\prime}\right)
$$

where $\varphi$ is a given element of $\widetilde{H}^{1 / 2}(\Gamma)$, the closure of $C_{0}^{\infty}(\Gamma)$ in $H^{1 / 2}\left(\mathbb{R}^{n-1}\right)$.

The energy of the harmonic field $U_{\varphi}$ is given by its Dirichlet integral, and Green's theorem yields

$$
\begin{aligned}
& \int_{\mathbb{R}^{n} \backslash \Gamma}\left|\nabla U_{\varphi}(\mathbf{x})\right|^{2} \mathrm{~d} \mathbf{x} \\
& =-\int_{\mathbb{R}^{n-1}} U_{\boldsymbol{\varphi}}\left(\mathbf{x}^{\prime},+0\right)\left(\frac{\partial}{\partial x_{n}} U_{\boldsymbol{\varphi}}\left(\mathbf{x}^{\prime},+0\right)\right) \mathrm{d} \mathbf{x}^{\prime}+\int_{\mathbb{R}^{n-1}} U_{\boldsymbol{\varphi}}\left(\mathbf{x}^{\prime},-0\right)\left(\frac{\partial}{\partial x_{n}} U_{\boldsymbol{\varphi}}\left(\mathbf{x}^{\prime},-0\right)\right) \mathrm{d} \mathbf{x}^{\prime} \\
& =\int_{\Gamma} \frac{1}{2} \varphi\left(\mathbf{x}^{\prime}\right)\left(\frac{\partial}{\partial x_{n}}(-\mathbf{W} \varphi)\left(\mathbf{x}^{\prime},-0\right)\right) \mathrm{d} \mathbf{x}^{\prime}-\int_{\Gamma} \frac{1}{2} \varphi\left(\mathbf{x}^{\prime}\right)\left(\frac{\partial}{\partial x_{n}}(-\mathbf{W} \varphi)\left(\mathbf{x}^{\prime},+0\right)\right) \mathrm{d} \mathbf{x}^{\prime} \\
& =-c_{n} \int_{\Gamma}\left\{\frac{1}{2} \varphi\left(\mathbf{x}^{\prime}\right) \frac{\partial}{\partial x_{n}}\left(\int_{\mathbb{R}^{n-1}} \frac{\left(\mathbf{x}^{\prime}-x_{n} \mathbf{e}_{n}-\mathbf{y}^{\prime}\right) \cdot \mathbf{e}_{n}}{\left|\mathbf{x}^{\prime}-x_{n} \mathbf{e}_{n}-\mathbf{y}^{\prime}\right|^{n}}\left(\boldsymbol{\varphi}\left(\mathbf{y}^{\prime}\right)-\boldsymbol{\varphi}\left(\mathbf{x}^{\prime}\right)\right) \mathrm{d} \mathbf{y}^{\prime}\right)\right. \\
& \left.+\frac{1}{2} \varphi\left(\mathbf{x}^{\prime}\right)^{2} \frac{\partial}{\partial x_{n}}\left(\int_{\mathbb{R}^{n-1}} \frac{\left(\mathbf{x}^{\prime}-x_{n} \mathbf{e}_{n}-\mathbf{y}^{\prime}\right) \cdot \mathbf{e}_{n}}{\left|\mathbf{x}^{\prime}-x_{n} \mathbf{e}_{n}-\mathbf{y}^{\prime}\right|^{n}} \mathrm{~d} \mathbf{y}^{\prime}\right)\right\} \mathrm{d} \mathbf{x}^{\prime} \\
& +c_{n}\left\{\frac{1}{2} \boldsymbol{\varphi}\left(\mathbf{x}^{\prime}\right) \frac{\partial}{\partial x_{n}}\left(\int_{\mathbb{R}^{n-1}} \frac{\left(\mathbf{x}^{\prime}+x_{n} \mathbf{e}_{n}-\mathbf{y}^{\prime}\right) \cdot \mathbf{e}_{n}}{\left|x^{\prime}+x_{n} \mathbf{e}_{n}-\mathbf{y}^{\prime}\right|^{n}}\left(\boldsymbol{\varphi}\left(\mathbf{y}^{\prime}\right)-\boldsymbol{\varphi}\left(\mathbf{x}^{\prime}\right)\right) \mathrm{d} \mathbf{y}^{\prime}\right)\right. \\
& \left.+\frac{1}{2} \varphi\left(\mathbf{x}^{\prime}\right)^{2} \frac{\partial}{\partial x_{n}}\left(\int_{\mathbb{R}^{n-1}} \frac{\left(\mathbf{x}^{\prime}+x_{n} \mathbf{e}_{n}-\mathbf{y}^{\prime}\right) \cdot \mathbf{e}_{n}}{\left|\mathbf{x}^{\prime}+x_{n} \mathbf{e}_{n}-\mathbf{y}^{\prime}\right|^{n}} \mathrm{~d} \mathbf{y}^{\prime}\right)\right\} \mathrm{d} \mathbf{x}^{\prime} .
\end{aligned}
$$

We can interchange differentiation and integration in this expression by means of Hadamard's finite part integral. Namely, due to

$$
\int_{\mathbb{R}^{n-1}} \frac{\left(\mathbf{x}^{\prime} \mp x_{n} \mathbf{e}_{n}-\mathbf{y}^{\prime}\right) \cdot \mathbf{e}_{n}}{\left|\mathbf{x}^{\prime} \mp x_{n} \mathbf{e}_{n}-\mathbf{y}^{\prime}\right|^{n}} \mathrm{~d} \mathbf{y}^{\prime}= \pm \frac{1}{2} \omega_{n}
$$


with the constant $\omega_{n}=\frac{1}{c_{n}}$, it holds

$$
\begin{aligned}
\int_{\mathbb{R}^{n} \backslash \Gamma}\left|\nabla U_{\boldsymbol{\varphi}}(\mathbf{x})\right|^{2} \mathrm{~d} \mathbf{x} & =(n-2) c_{n} \int_{\Gamma} \varphi\left(\mathbf{x}^{\prime}\right) \int_{\Gamma}\left|\mathbf{x}^{\prime}-\mathbf{y}^{\prime}\right|^{-n}\left(\varphi\left(\mathbf{y}^{\prime}\right)-\boldsymbol{\varphi}\left(\mathbf{x}^{\prime}\right)\right) \mathrm{d} \mathbf{y}^{\prime} \mathrm{d} \mathbf{x}^{\prime} \\
& =(n-2) \int_{\Gamma} \boldsymbol{\varphi}\left(\mathbf{x}^{\prime}\right)(\mathbf{D} \boldsymbol{\varphi})\left(\mathbf{x}^{\prime}\right) \mathrm{d} \mathbf{x}^{\prime},
\end{aligned}
$$

where

$$
\mathbf{D} \boldsymbol{\varphi}\left(\mathbf{x}^{\prime}\right)=- \text { p.f. } c_{n} \int_{\Gamma}\left(\frac{\partial}{\partial x_{n}} \frac{\partial}{\partial y_{n}}|\mathbf{x}-\mathbf{y}|^{2-n}\right) \boldsymbol{\varphi}\left(\mathbf{y}^{\prime}\right) \mathrm{d} \mathbf{y}^{\prime}
$$

is Hadamard's finite part integral with $\mathbf{x}=\left(\mathbf{x}^{\prime}, 0\right), \mathbf{y}=\left(\mathbf{y}^{\prime}, 0\right)$ and with the hypersingular kernel function $k_{\mathbf{D}}(\mathbf{x}, \mathbf{y})=(n-2)\left|\mathbf{x}^{\prime}-\mathbf{y}^{\prime}\right|^{-n} c_{n}$, that is

$$
\int_{\mathbb{R}^{n} \backslash \Gamma}\left|\nabla U_{\boldsymbol{\varphi}}(\mathbf{x})\right|^{2} \mathrm{~d} \mathbf{x}=(n-2) c_{n} \int_{\Gamma} \text { p.f. } \int_{\Gamma}\left|\mathbf{x}^{\prime}-\mathbf{y}^{\prime}\right|^{-n} \boldsymbol{\varphi}\left(\mathbf{x}^{\prime}\right) \boldsymbol{\varphi}\left(\mathbf{y}^{\prime}\right) \mathrm{d} \mathbf{x}^{\prime} \mathrm{d} \mathbf{y}^{\prime} .
$$

(For the definition of Hadamard's partie finie integral operators, see [7] and [12, Chapter 3.2].)

Hence, the finite part integral on the right of (2.1) which has the Riesz kernel $\left|\mathbf{x}^{\prime}-\mathbf{y}^{\prime}\right|^{-n}$ for $\mathbf{x}^{\prime}, \mathbf{y}^{\prime} \in \Gamma \subset \mathbb{R}^{n-1}$ defines the energy of the harmonic double layer potential in $\mathbb{R}^{n} \backslash \Gamma$ given by the Dirichlet integral on the left of (2.1). Since the Riesz kernel is a homogeneous function of degree $-n$, it defines on $\Gamma \subset \mathbb{R}^{n-1}$ a strongly elliptic pseudodifferential operator $\mathbf{D}$ of order 1 (see [12, Section 7.1.2]).

\section{Strongly singular Riesz EnERgy on A MANifold}

In all that follows, without stated otherwise, we fix $n \geq 2$ and $-1<\alpha<1$, and write $\beta:=1-\alpha$.

In $\mathbb{R}^{n}$, consider a strongly singular Riesz kernel $|\mathbf{x}-\mathbf{y}|^{\alpha-n}$ and a manifold $\Gamma:=\bigcup_{\ell \in I} \Gamma_{\ell}$, where $\Gamma_{\ell}$ are finitely many compact, connected, mutually disjoint, boundaryless, $(n-1)$-dimensional, oriented $C^{\infty}$-manifolds, immersed into $\mathbb{R}^{n}$. Then, the surface measure $\mathrm{d} s$ on $\Gamma$ is well defined.

In what follows, $(\boldsymbol{\psi}, \boldsymbol{\varphi})_{L^{2}(\Gamma)}$ will stand for the extension of the $L^{2}$-scalar product to dualities as $\boldsymbol{\psi} \in H^{-\beta / 2}(\Gamma)$ and $\varphi \in H^{\beta / 2}(\Gamma)$ and also to the applications of distributions $\boldsymbol{\psi}$ on $\Gamma$ operating on $\varphi \in C^{\infty}(\Gamma)$.

We call the strongly singular partie finie integral of the Riesz kernel

$$
\left(V_{\beta} \boldsymbol{\varphi}, \varphi\right)_{L_{2}(\Gamma)}=\int_{\Gamma} \text { p.f. } \int_{\Gamma}|\mathbf{x}-\mathbf{y}|^{\alpha-n} \boldsymbol{\varphi}(\mathbf{x}) \varphi(\mathbf{y}) \mathrm{d} s_{\mathbf{x}} \mathrm{d} s_{\mathbf{y}}=: E_{\alpha}(\boldsymbol{\varphi})
$$

with respect to $|\mathbf{x}-\mathbf{y}| \geq \varepsilon_{0} \rightarrow 0, \varepsilon_{0}>0$, operating on $\varphi \in C^{\infty}(\Gamma)$, the energy of the Riesz potential

$$
U_{\varphi}(\mathbf{x})=\text { p.f. } \int_{\mathbf{y} \in \Gamma}|\mathbf{x}-\mathbf{y}|^{\alpha-n} \varphi(\mathbf{y}) \mathrm{d} s_{\mathbf{y}}, \quad \mathbf{x} \in \mathbb{R}^{n},
$$

generated by the surface charge $\varphi$ (see e.g. [12]). For $\varphi \in C^{\infty}(\Gamma)$, the Hadamard partie finie integral operator

$$
V_{\beta} \boldsymbol{\varphi}(\mathbf{x})=\text { p.f. } \int_{\mathbf{y} \in \Gamma}|\mathbf{x}-\mathbf{y}|^{\alpha-n} \boldsymbol{\varphi}(\mathbf{y}) \mathrm{d} s_{\mathbf{y}}, \quad \mathbf{x} \in \Gamma,
$$

which underlies (3.1), is for $0 \leq \alpha<1$ given by

$$
V_{\beta} \boldsymbol{\varphi}(\mathbf{x})=\text { p.v. } \int_{\mathbf{y} \in \Gamma \wedge|\mathbf{x}-\mathbf{y}|>0}|\mathbf{x}-\mathbf{y}|^{-\beta-(n-1)}\{\boldsymbol{\varphi}(\mathbf{y})-\boldsymbol{\varphi}(\mathbf{x})\} \mathrm{d} s_{\mathbf{y}}+h(\mathbf{x}) \boldsymbol{\varphi}(\mathbf{x}),
$$

where

$$
h(\mathbf{x})=\text { p.f. } \lim _{\delta \rightarrow 0} \int_{\mathbf{y} \in \Gamma \wedge 0<\delta<|\mathbf{x}-\mathbf{y}|}|\mathbf{x}-\mathbf{y}|^{-\beta-(n-1)} \mathrm{d} s_{\mathbf{y}},
$$


and for $-1<\alpha<0$ by

$$
\begin{gathered}
V_{\beta} \boldsymbol{\varphi}(\mathbf{x})=\text { p.v. } \int_{\mathbf{y} \in \Gamma \wedge|\mathbf{x}-\mathbf{y}|>0}|\mathbf{x}-\mathbf{y}|^{-\beta-(n-1)}\{\boldsymbol{\varphi}(\mathbf{y})-\boldsymbol{\varphi}(\mathbf{x})-(\mathbf{y}-\mathbf{x}) \cdot \nabla \boldsymbol{\varphi}(\mathbf{x})\} \mathrm{d} s_{\mathbf{y}} \\
+h(\mathbf{x}) \boldsymbol{\varphi}(\mathbf{x})+\mathbf{h}(\mathbf{x}) \cdot \nabla \boldsymbol{\varphi}(\mathbf{x}),
\end{gathered}
$$

where

$$
\mathbf{h}(\mathbf{x})=\text { p.f. } \lim _{\delta \rightarrow 0} \int_{\mathbf{y} \in \Gamma \wedge 0<\delta<|\mathbf{x}-\mathbf{y}|}|\mathbf{x}-\mathbf{y}|^{-\beta-(n-1)}(\mathbf{y}-\mathbf{x}) \mathrm{d} s \mathbf{y} .
$$

The abbrevation p.v. stands for the Calderon-Mikhlin principal value integral (see [17]). (See Appendix A for the explicit computation of the partie finie integrals $h(\mathbf{x})$ and $\mathbf{h}(\mathbf{x})$.)

Theorem 3.1 (see [12, Chapter 8]). The partie finie integral operator $V_{\beta}$ is a strongly elliptic pseudodifferential operator of order $\beta=1-\alpha \in(0,2)$ on $\Gamma$. The principal symbol of this operator is given by the equivalence class associated with the homogeneous function

$$
a(\boldsymbol{\xi})=C(n-1, \beta)|\boldsymbol{\xi}|^{\beta}, \text { where } C(n-1, \beta)=2^{-\beta} \pi^{\frac{n-1}{2}} \frac{\Gamma\left(\frac{-\beta}{2}\right)}{\Gamma\left(\frac{n-\alpha}{2}\right)} \text { and } \xi \in \mathbb{R}^{n-1} .
$$

$V_{\beta}$ defines the linear and continuous mapping $V_{\beta}: H^{s}(\Gamma) \rightarrow H^{s-\beta}(\Gamma)$ for every $s \in \mathbb{R}$. In particular, for $s=\beta / 2, V_{\beta}$ maps $H^{\beta / 2}(\Gamma)$ into $H^{-\beta / 2}(\Gamma)$ and there exist $0<c_{0} \leq c_{2}$ and $c_{1} \geq 0$ such that the inequalities

$$
c_{0}\|\boldsymbol{\varphi}\|_{H^{\beta / 2}(\Gamma)}^{2}-c_{1}\|\boldsymbol{\varphi}\|_{L^{2}(\Gamma)}^{2} \leq\left(V_{\beta} \boldsymbol{\varphi}, \boldsymbol{\varphi}\right)_{L_{2}(\Gamma)} \leq c_{2}\|\boldsymbol{\varphi}\|_{H^{\beta / 2}(\Gamma)}^{2}
$$

are satisfied for any $\varphi \in H^{\beta / 2}(\Gamma)$.

Proof. For justifying the inequalities (3.5), recall that for each of the components of the $C^{\infty_{-}}$ manifolds $\Gamma_{\ell}, \ell \in I$, immersed into $\mathbb{R}^{n}$, we may associate a family of finite-dimensional atlases $\mathfrak{A}_{\ell}$ (see [13]). Each atlas $\mathfrak{A}_{\ell}$ is a family of local charts $\left(O_{\ell r}, \mathcal{U}_{\ell r}, \mathcal{X}_{\ell r}\right)$, where $r$ ranges through a finite set $R_{\ell}$. The open sets $O_{\ell r} \subset \Gamma_{\ell}$ define an open covering of $\Gamma_{\ell}$, while $\mathcal{X}_{\ell r}$ is a $C^{\infty}$-diffeomorphism of $O_{\ell r}$ onto $\mathcal{U}_{\ell r} \subset \mathbb{R}^{n-1}$. Let $\left\{\beta_{\ell r}\right\}_{r \in R_{\ell}}$ be a $C^{\infty}$-partition of unity of $\Gamma_{\ell}$ which is subordinate to the atlas $\mathfrak{A}_{\ell}$. In addition to the partition of unity, let $\left\{\gamma_{\ell r}\right\}_{r \in R_{\ell}}$ be a second system of functions $\gamma_{\ell r} \in C_{0}^{\infty}\left(O_{\ell r}\right)$ with the properties

$$
\gamma_{\ell r}(\mathbf{x})=1 \text { for all } \mathbf{x} \in \operatorname{supp} \beta_{\ell r} \text { and } 0 \leq \gamma_{\ell r} .
$$

Thus, it holds that

$$
\gamma_{\ell r}(\mathbf{x}) \beta_{\ell r}(\mathbf{x})=\beta_{\ell r}(\mathbf{x}) \text { and } \beta_{\ell r}(\mathbf{x}) \gamma_{\ell r}(\mathbf{x})=\beta_{\ell r}(\mathbf{x}) \text { for all } \mathbf{x} \in \Gamma_{\ell} .
$$

With respect to the atlas $\mathfrak{A}_{\ell}$, let $\mathcal{X}_{\ell r \star}$ denote the corresponding pushforwards and $\mathcal{X}_{\ell r}^{\star}$ the pullbacks. Then $\mathcal{X}_{\ell r \star} \beta_{\ell r} \in C_{0}^{\infty}\left(\mathcal{U}_{\ell r}\right)$.

Without loss of generality, the local parametric representations can always be chosen in such a way that at one point $\mathbf{x}_{\ell r}^{\circ} \in O_{\ell r}$ where $\beta_{\ell r}\left(\mathbf{x}_{\ell r}^{\circ}\right)=1$ we have $\mathcal{X}_{\ell r}\left(\mathbf{x}_{\ell r}^{\circ}\right)=\mathbf{0}$ and, moreover, at this point the tangent bundle

$$
\left.\frac{\partial \mathbf{x}}{\partial \mathbf{x}^{\prime}}\right|_{\mathbf{x}^{\prime}=\mathbf{0}}=\left.\frac{\partial \mathcal{X}_{\ell r}^{-1}\left(\mathbf{x}^{\prime}\right)}{\partial \mathbf{x}^{\prime}}\right|_{\mathbf{x}^{\prime}=\mathbf{0}}, \text { where } \mathbf{x}^{\prime}:=\mathcal{X}_{\ell r}(\mathbf{x})
$$

forms a positively oriented system of $n-1$ mutually orthogonal unit vectors. This implies that the Riemannian tensor of $\Gamma_{\ell}$ in the local coordinates at the point $\mathbf{x}_{\ell r}^{\circ}$ is the unity matrix. Hence, the surface measure satisfies

$$
\mathrm{d} s_{\ell}(\mathbf{x})=J_{\ell r}\left(\mathbf{x}^{\prime}\right) \mathrm{d} \mathbf{x}^{\prime} \text { where } \mathbf{x}^{\prime} \in \mathcal{U}_{\ell r}, \quad \text { and } J_{\ell r}(\mathbf{0})=\mathbf{1} .
$$

Given an atlas $\mathfrak{A}_{\ell}$ on $\Gamma_{\ell}$, define

$$
d_{\ell}:=\min _{r \in R_{\ell}} \operatorname{diam} \mathcal{U}_{\ell r}
$$

Thus, one can choose $\delta_{0}>0$ so that for any given $0<\delta<\delta_{0}$ there exists a finite-dimensional atlas $\mathfrak{A}_{\ell}^{\delta}$ satisfying all the above formulated properties and $d_{\ell}=\delta$. Hence, we have a whole family 
of finite atlases $\mathfrak{A}_{\ell}^{\delta}$ with $0<\delta<\delta_{0}$ which will be under consideration. (We then shall omit the index $\delta$ in the notation.)

Note that the Jacobians $J_{\ell r}$ depend on the geometric properties of $\Gamma_{\ell}$ only, and $J_{\ell r}$ together with their derivatives are uniformly continuous relative to $\delta \in\left(0, \delta_{0}\right)$.

Corresponding to the partition of unity, the pseudodifferential operator $V_{\beta}$ on $\Gamma$ can be decomposed as

$$
V_{\beta}=\sum_{\ell \in I} \sum_{r \in R_{\ell}} \beta_{\ell r} V_{\beta} \gamma_{\ell r}+Z_{1}=\sum_{\ell \in I} \sum_{r \in R_{\ell}} \gamma_{\ell r} V_{\beta} \beta_{\ell r}+Z_{2}
$$

and

$$
V_{\beta}=\sum_{\ell \in I} \sum_{r \in R_{\ell}} \beta_{\ell r} \mathcal{X}_{\ell r}^{\star} V_{\ell r} \mathcal{X}_{\ell r \star} \gamma_{\ell r}+Z_{1}=\sum_{\ell \in I} \sum_{r \in R_{\ell}} \gamma_{\ell r} \mathcal{X}_{\ell r}^{\star} V_{\ell r} \mathcal{X}_{\ell r \star} \beta_{\ell r}+Z_{2}
$$

Herein, $Z_{1}, Z_{2}$ are smoothing operators of order $-\infty$ in view of $|\mathbf{x}-\mathbf{y}|^{\alpha-n} \in C^{\infty}\left(\Gamma_{p} \times \Gamma_{k}\right)$ for $p \neq k$ and $\operatorname{supp}\left(1-\gamma_{\ell r}\right) \cap \operatorname{supp}\left(\beta_{\ell r}\right)=\emptyset$ if $\mathbf{x}, \mathbf{y} \in \Gamma_{\ell}$ (see also [12, Chapter 8]). Moreover,

$$
V_{\ell r} \boldsymbol{\varphi}\left(\mathbf{x}^{\prime}\right)=\text { p.f. } \int_{\mathcal{U}_{\ell r}}\left|\mathcal{X}_{\ell r}^{-1}\left(\mathbf{x}^{\prime}\right)-\mathcal{X}_{\ell r}^{-1}\left(\mathbf{y}^{\prime}\right)\right|^{\alpha-n} \boldsymbol{\varphi}\left(\mathbf{y}^{\prime}\right) J_{\ell r}\left(\mathbf{y}^{\prime}\right) \mathrm{d} \mathbf{y}^{\prime}, \varphi \in C_{0}^{\infty}\left(\mathcal{U}_{\ell r}\right)
$$

are the localized operators in the parametric domains $\mathcal{U}_{\ell r}$, defined by the operator $V_{\beta}$.

The inequalities (3.5) now follow locally on each chart of the atlas $\mathfrak{A}_{\ell}$ for the localized operators $V_{\ell r}$ in local coordinates in $\mathcal{U}_{\ell r}$. With Martensen's surface polar coordinates ((B.5), (B.9) in Appendix B), the kernel of $V_{\ell r}$ admits a pseudohomogeneous asymptotic expansion of the form

$$
k\left(\mathbf{x}^{\prime}, \varrho\right)=\varrho^{-\beta-(n-1)}\left\{1+\sum_{j \geq 2} k_{\ell r, j}\left(\mathbf{x}^{\prime}, \varrho\right)\right\},
$$

where

$$
k_{\ell r, j}\left(\mathbf{x}^{\prime}, t \varrho\right)=t^{j} k_{\ell r, j}\left(\mathbf{x}^{\prime}, \varrho\right) \text { for } t>0, \varrho>0
$$

since $\left|\mathbf{x}^{\prime}-\mathbf{x}_{0}^{\prime}\right|^{2}$ satisfies the expansion (B9). Correspondingly, the symbol $a\left(\mathbf{x}^{\prime}, \boldsymbol{\xi}^{\prime}\right)$ of $V_{\ell r}$ has the asymptotic expansion

$$
a\left(\mathbf{x}^{\prime}, \boldsymbol{\xi}^{\prime}\right)=\left|\boldsymbol{\xi}^{\prime}\right|^{\beta}\left\{1+\sum_{j \geq 2} a_{-j}^{0}\left(\mathbf{x}^{\prime}, \boldsymbol{\xi}^{\prime}\right)\right\},
$$

where $a_{-j}^{0}\left(\mathbf{x}^{\prime}, t \boldsymbol{\xi}^{\prime}\right)=t^{-j} a_{-j}^{0}\left(\mathbf{x}^{\prime}, \boldsymbol{\xi}^{\prime}\right)$ with $t>0, \boldsymbol{\xi}^{\prime} \neq \mathbf{0}$.

Then, Fourier transform and Parseval's theorem yield

$$
\left(V_{\ell r} \boldsymbol{\varphi}, \boldsymbol{\varphi}\right)_{L_{2}\left(\mathcal{U}_{\ell r}\right)}=\int_{\mathbb{R}^{n-1}}\left|\boldsymbol{\xi}^{\prime}\right|^{\beta}\left|\widehat{\boldsymbol{\varphi}}\left(\boldsymbol{\xi}^{\prime}\right)\right|^{2} \mathrm{~d} \boldsymbol{\xi}^{\prime}+\left(A_{\ell r}^{\beta-2} \boldsymbol{\varphi}, \boldsymbol{\varphi}\right)_{L_{2}\left(\mathcal{U}_{\ell r}\right)}+\left(R_{\ell r} \boldsymbol{\varphi}, \boldsymbol{\varphi}\right)_{L_{2}\left(\mathcal{U}_{\ell r}\right)},
$$

where $A_{\ell r}^{\beta-2}$ is a pseudodifferential operator of order $\beta-2$ :

$$
\left(A_{\ell r}^{\beta-2} \boldsymbol{\varphi}\right)\left(\mathbf{x}^{\prime}\right)=\int_{\mathbb{R}^{n-1}} e^{i \mathbf{x}^{\prime} \cdot \boldsymbol{\xi}^{\prime}} \Psi_{\ell r}\left(\boldsymbol{\xi}^{\prime}\right)\left|\boldsymbol{\xi}^{\prime}\right|^{\beta} \sum_{j \geq 2} a_{-j}^{0}\left(\mathbf{x}^{\prime}, \boldsymbol{\xi}^{\prime}\right) \widehat{\boldsymbol{\varphi}}\left(\boldsymbol{\xi}^{\prime}\right) \mathrm{d} \boldsymbol{\xi}^{\prime} .
$$

Here, the function $\boldsymbol{\Psi}_{\ell r} \in C^{\infty}\left(\mathbb{R}^{n-1}\right)$ is arbitrary but fixed such that $0 \leq \boldsymbol{\Psi}_{\ell r}\left(\boldsymbol{\xi}^{\prime}\right) \leq 1$ and

$$
\boldsymbol{\Psi}_{\ell r}\left(\boldsymbol{\xi}^{\prime}\right)=\mathbf{0} \text { for }\left|\boldsymbol{\xi}^{\prime}\right| \leq \frac{1}{2} \text { and } \boldsymbol{\Psi}_{\ell r}\left(\boldsymbol{\xi}^{\prime}\right)=1 \text { for }\left|\boldsymbol{\xi}^{\prime}\right| \geq 1 \text {. }
$$

The remainder operator $R_{\ell r} \varphi(\mathbf{x})=\int_{\Gamma} R_{\ell r}(\mathbf{x}, \mathbf{y}) \varphi(\mathbf{y}) \mathrm{d} s_{\mathbf{y}}$ is a smoothing operator with the smooth kernel function $R_{\ell r} \in C^{\infty}(\Gamma \times \Gamma)$. Hence, there exists a constant $c_{\ell r}>0$ such that

$$
\left(V_{\ell r} \boldsymbol{\varphi}, \boldsymbol{\varphi}\right)_{L_{2}\left(\mathcal{U}_{\ell r}\right)} \leq c_{\ell r} \int_{\mathbb{R}^{n-1}}\left(1+\left|\boldsymbol{\xi}^{\prime}\right|\right)^{\beta}\left|\widehat{\boldsymbol{\varphi}}\left(\boldsymbol{\xi}^{\prime}\right)\right|^{2} \mathrm{~d} \boldsymbol{\xi}^{\prime}=c\|\boldsymbol{\varphi}\|_{H^{\beta / 2}\left(\mathcal{U}_{\ell r}\right)}^{2}
$$

which implies with some constant $c_{2}>0$ that

$$
\left(V_{\beta} \boldsymbol{\varphi}, \boldsymbol{\varphi}\right)_{L_{2}(\Gamma)} \leq c_{2}\|\boldsymbol{\varphi}\|_{H^{\beta / 2}(\Gamma)}^{2} .
$$


Vice versa, from

$$
\left(V_{\beta} \boldsymbol{\varphi}, \boldsymbol{\varphi}\right)_{L_{2}\left(\mathcal{U}_{\ell r}\right)} \geq \int_{\mathbb{R}^{n-1}}\left(1+\left|\boldsymbol{\xi}^{\prime}\right|\right)^{\beta} \mid \widehat{\boldsymbol{\varphi}}\left(\left.\boldsymbol{\xi}^{\prime}\right|^{2} \mathrm{~d} \boldsymbol{\xi}^{\prime}-c_{\ell r}^{\prime} \int_{\mathbb{R}^{n-1}}\left(1+\left|\boldsymbol{\xi}^{\prime}\right|\right)^{\beta-2}\left|\widehat{\boldsymbol{\varphi}}\left(\boldsymbol{\xi}^{\prime}\right)\right|^{2} \mathrm{~d} \boldsymbol{\xi}^{\prime},\right.
$$

after summation over $\ell \in I$, we obtain the Gårding inequality

$$
\left(V_{\beta} \boldsymbol{\varphi}, \boldsymbol{\varphi}\right)_{L_{2}(\Gamma)} \geq c_{0}\|\boldsymbol{\varphi}\|_{H^{\beta / 2}(\Gamma)}^{2}-c_{1}\|\boldsymbol{\varphi}\|_{L_{2}(\Gamma)}^{2} .
$$

The embedding $H^{\beta / 2}(\Gamma) \hookrightarrow L_{2}(\Gamma)$ is compact since $0<\beta<2$. For $\beta=1 \in \mathbb{N}_{0}$ the Tricomi condition needs to be satisfied for $V_{\beta}$ being a pseudodifferential operator and reads here as

$$
\int_{|\Theta|=1} \Theta^{\boldsymbol{\alpha}^{\prime}} \mathrm{d} \omega(\Theta)=0 \text { for }\left|\boldsymbol{\alpha}^{\prime}\right|=1
$$

(see $\left[12\right.$, Theorem 7.1.7]). In addition, we have $a_{-1}^{0}\left(\mathbf{x}^{\prime}, \boldsymbol{\xi}^{\prime}\right)=0$. So, $V_{\beta}$ is a classical pseudodifferential operator on $\Gamma$ of order $\beta \in(0,2)$.

Now, we introduce a set of so-called admissible measures or charges located on $\Gamma$. Recall that $\Gamma=\bigcup_{\ell \in I} \Gamma_{\ell}$, where the finitely many $\Gamma_{\ell}$ are compact, nonintersecting, boundaryless, connected, $(n-1)$-dimensional, orientable $C^{\infty}$-manifolds, immersed into $\mathbb{R}^{n}$. With each $\Gamma_{\ell}$ we associate a prescribed sign $\alpha_{\ell} \in\{-1,1\}$ where $\alpha_{\ell}=+1$ for $\ell \in I^{+}$and $\alpha_{\ell}=-1$ for $\ell \in I^{-}$. Then $I=I^{+} \cup I^{-}$ and $I^{+} \cap I^{-}=\emptyset, I^{-}=\emptyset$ is admitted. Let $\mathfrak{M}(\Gamma)$ denote the $\sigma$-algebra of signed Borel measures $\boldsymbol{\nu}$ on $\Gamma$ equipped with the topology of pointwise convergence on $C(\Gamma)$, the class of all real-valued continuous functions on $\Gamma$.

Next, consider the manifold $\Gamma$ being loaded by charges of the form

$$
\boldsymbol{\mu}=\sum_{\ell \in I} \alpha_{\ell} \mu^{\ell}
$$

where, for every $\ell \in I, \mu^{\ell}$ is a nonnegative Borel measure on $\Gamma_{\ell}$. The convex cone of all signed measures $\boldsymbol{\mu}$ of the form (3.10) will be denoted by $\mathfrak{M}^{+}(\Gamma)$.

The following theorem deals with absolutely continuous $\boldsymbol{\Sigma} \in \mathfrak{M}^{+}(\Gamma)$, i.e. $\mathrm{d} \boldsymbol{\Sigma}=\boldsymbol{\sigma}$ d $s$, with densities $\boldsymbol{\sigma} \in \mathcal{K}^{\beta / 2}(\Gamma)$,

$$
\mathcal{K}^{\beta / 2}(\Gamma):=\left\{\boldsymbol{\sigma}=\sum_{\ell \in I} \alpha_{\ell} \sigma^{\ell}, \text { where } \sigma^{\ell} \in H^{\beta / 2}\left(\Gamma_{\ell}\right) \text { and } \sigma^{\ell} \geq 0\right\} .
$$

For brevity, we shall often identify an absolutely continuous Borel measure $\boldsymbol{\Sigma} \in \mathfrak{M}^{+}(\Gamma)$ with $\boldsymbol{\sigma}$, its density. Likewise, the cone of all $\boldsymbol{\Sigma} \in \mathfrak{M}^{+}(\Gamma)$ with $\boldsymbol{\sigma} \in \mathcal{K}^{\beta / 2}(\Gamma)$ will be denoted by $\mathcal{K}^{\beta / 2}(\Gamma)$, provided that this will not cause any misunderstanding. Similar to as it has been done in (3.1), we define the Riesz energy of $\boldsymbol{\Sigma}=\boldsymbol{\sigma} \in \mathcal{K}^{\beta / 2}(\Gamma)$ by

$$
E_{\alpha}(\boldsymbol{\Sigma}):=\left(V_{\beta} \boldsymbol{\sigma}, \boldsymbol{\sigma}\right)_{L_{2}(\Gamma)}=\int_{\Gamma} \text { p.f. } \int_{\Gamma}|\mathbf{x}-\mathbf{y}|^{\alpha-n} \mathrm{~d} \boldsymbol{\Sigma}(\mathbf{x}) \mathrm{d} \boldsymbol{\Sigma}(\mathbf{y}) .
$$

Theorem 3.2. For any $\boldsymbol{\Sigma}=\boldsymbol{\sigma} \in \mathcal{K}^{\beta / 2}(\Gamma)$, the Riesz energy is finite and satisfies the inequalities

$$
c_{0}^{\prime}\|\boldsymbol{\sigma}\|_{H^{\beta / 2(\Gamma)}}^{2} \leq E_{\alpha}(\boldsymbol{\Sigma}):=\left(V_{\beta} \boldsymbol{\sigma}, \boldsymbol{\sigma}\right)_{L_{2}(\Gamma)} \leq c_{1}^{\prime}\|\boldsymbol{\sigma}\|_{H^{\beta / 2(\Gamma)}}^{2},
$$

the constants $c_{0}^{\prime}$ and $c_{1}^{\prime}$ being strictly positive and independent of $\boldsymbol{\Sigma}$. This means that $V_{\beta}$ is continuously invertible on $\mathcal{K}^{\beta / 2}(\Gamma)$.

Proof. Write

$$
\mathcal{K}^{\infty}(\Gamma):=\left\{\boldsymbol{\sigma}=\sum_{\ell \in I} \alpha_{\ell} \sigma^{\ell}, \text { where } \sigma^{\ell} \in C^{\infty}\left(\Gamma_{\ell}\right) \text { and } \sigma^{\ell} \geq 0 \text { on } \Gamma_{\ell}\right\} .
$$


Let $\psi(\varrho)$ be a $C_{0}^{\infty}(\mathbb{R})$-function with $\psi(\varrho)=1$ for $0 \leq \varrho \leq \delta, 0 \leq \psi(\varrho) \leq 1$ and $\psi(\varrho)=0$ for $\varrho \geq 2 \delta>0$. Having the atlas $\mathfrak{A}_{\ell}^{\delta}$ at hand, we use the decomposition (3.6) and the representation in local coordinates (3.7) to arrive at

$$
\begin{aligned}
\left(V_{\ell r} \boldsymbol{\sigma}\right)\left(\mathbf{x}^{\prime}\right)= & \text { p.f. } \int_{\mathbb{R}^{n-1}}\left|\mathbf{x}^{\prime}-\mathbf{y}^{\prime}\right|^{-\beta-(n-1)} \psi_{\star}\left(\left|\mathbf{x}^{\prime}-\mathbf{y}^{\prime}\right|\right) \boldsymbol{\sigma}_{\star}\left(\mathbf{y}^{\prime}\right) J_{\ell r}\left(\mathbf{y}^{\prime}\right) \mathrm{d} \mathbf{y}^{\prime} \\
& +\int_{\left|\mathbf{x}^{\prime}-\mathbf{y}^{\prime}\right| \geq \delta}\left|\mathbf{x}^{\prime}-\mathbf{y}^{\prime}\right|^{-\beta-(n-1)}\left(1-\psi_{\star}\left(\left|\mathbf{x}^{\prime}-\mathbf{y}^{\prime}\right|\right)\right) \boldsymbol{\sigma}_{\star}\left(\mathbf{y}^{\prime}\right) J_{\ell r}\left(\mathbf{y}^{\prime}\right) \mathrm{d} \mathbf{y}^{\prime},
\end{aligned}
$$

where $\psi_{\star}$ and $\boldsymbol{\sigma}_{\star}$ are the pushforwards of $\psi$ and $\boldsymbol{\sigma}$, respectively. Summation gives

$$
\begin{aligned}
&\left(\boldsymbol{\sigma}, V_{\beta} \boldsymbol{\sigma}\right)_{L_{2}(\Gamma)}= \sum_{\ell, i \in I} \sum_{r \in R_{\ell}} \sum_{q \in R_{i}} \int_{\mathbb{R}^{n-1}} \text { p.f. } \int_{\mathbb{R}^{n-1}}\left|\mathbf{x}^{\prime}-\mathbf{y}^{\prime}\right|^{-\beta-(n-1)} \psi_{\star}\left(\left|\mathbf{x}^{\prime}-\mathbf{y}^{\prime}\right|\right) \\
& \cdot \boldsymbol{\sigma}_{\star}\left(\mathbf{y}^{\prime}\right) J_{\ell r}\left(\mathbf{y}^{\prime}\right) \mathrm{d} \mathbf{y}^{\prime} \boldsymbol{\sigma}_{\star}\left(\mathbf{x}^{\prime}\right) J_{i q}\left(\mathbf{x}^{\prime}\right) \mathrm{d} \mathbf{x}^{\prime} \\
&+\iint_{|\mathbf{x}-\mathbf{y}| \geq \delta}|\mathbf{x}-\mathbf{y}|^{-\beta-(n-1)}(1-\psi(|\mathbf{x}-\mathbf{y}|)) \boldsymbol{\sigma}(\mathbf{y}) \mathrm{d} s_{\mathbf{y}} \boldsymbol{\sigma}(\mathbf{x}) \mathrm{d} s_{\mathbf{x}} .
\end{aligned}
$$

Since the localized operators $V_{\ell r}$ are all pseudodifferential operators of order $\beta$ with positive definite principal symbol (3.4), hence strongly elliptic, one finds with Fourier transform and Parseval's theorem the estimate

$$
\begin{gathered}
\sum_{\ell, i \in I} \sum_{r \in R_{\ell}} \sum_{q \in R_{i}} \int_{\mathbb{R}^{n-1}} \text { p.f. } \int_{\mathbb{R}^{n-1}}\left|\mathbf{x}^{\prime}-\mathbf{y}^{\prime}\right|^{-\beta-(n-1)} \psi_{\star}\left(\left|\mathbf{x}^{\prime}-\mathbf{y}^{\prime}\right|\right) \\
\cdot \boldsymbol{\sigma}_{\star}\left(\mathbf{y}^{\prime}\right) J_{\ell r}\left(\mathbf{y}^{\prime}\right) \mathrm{d} \mathbf{y}^{\prime} \boldsymbol{\sigma}_{\star}\left(\mathbf{x}^{\prime}\right) J_{i q}\left(\mathbf{x}^{\prime}\right) \mathrm{d} \mathbf{x}^{\prime} \\
\geq c_{0}^{\prime}\|\boldsymbol{\sigma}\|_{H^{\beta / 2}(\Gamma)}^{2}-c^{\prime \prime} \frac{1}{2-\beta} \delta^{2-\beta}\|\boldsymbol{\sigma}\|_{L_{2}(\Gamma)}^{2} \geq c_{0}^{\prime \prime \prime}\|\boldsymbol{\sigma}\|_{H^{\beta / 2}(\Gamma)}^{2}
\end{gathered}
$$

with $c_{0}^{\prime \prime \prime}>0$ since $H^{\beta / 2}(\Gamma) \hookrightarrow L_{2}(\Gamma)$ compactly and if $\delta>0$ is chosen sufficiently small. The remaining quadratic form

$$
\iint_{|\mathbf{x}-\mathbf{y}| \geq \delta}|\mathbf{x}-\mathbf{y}|^{-\beta-(n-1)}(1-\psi(|\mathbf{x}-\mathbf{y}|)) \boldsymbol{\sigma}(\mathbf{y}) \mathrm{d} s_{\mathbf{y}} \boldsymbol{\sigma}(\mathbf{x}) \mathrm{d} s_{\mathbf{x}}
$$

has a strictly positive $C^{\infty}$-kernel. Hence the left inequality in (3.11) is satisfied for $\sigma \in \mathcal{K}^{\infty}(\Gamma)$, $\boldsymbol{\sigma} \not \equiv \mathbf{0}$. Since $\mathcal{K}^{\infty}(\Gamma)$ is dense in $\mathcal{K}^{\beta / 2}(\Gamma)$, the left inequality in (3.11) also holds for $\boldsymbol{\sigma} \in \mathcal{K}^{\beta / 2}(\Gamma)$, $\boldsymbol{\sigma} \not \equiv \mathbf{0}$ by completion. The right inequality in (3.11) was already shown in (3.9) for $\boldsymbol{\sigma}$ in place of $\boldsymbol{\varphi}$. This completes the proof.

We next proceed by defining the notion of the Riesz energy for arbitrary (not necessarily absolutely continuous) measures $\boldsymbol{\Sigma} \in \mathfrak{M}^{+}(\Gamma)$. Since $V_{\beta}$ is a classical pseudodifferential operator on $\Gamma$, it maps the distribution given by the Radon measure $\boldsymbol{\Sigma} \in \mathfrak{M}^{+}(\Gamma)$ to $V_{\beta} \boldsymbol{\Sigma}$ which is a distribution again, and this linear mapping is continuous in the weak topology of distributions (see Theorem II.1.5 in [20]). Therefore, the action of the measure $V_{\beta} \boldsymbol{\Sigma}$ on functions $\varphi \in C^{\infty}(\Gamma)$ is well defined and

since $V_{\beta}$ is symmetric.

$$
\left(V_{\beta} \boldsymbol{\Sigma}, \boldsymbol{\varphi}\right)_{L_{2}(\Gamma)}=\left(\boldsymbol{\Sigma}, V_{\beta} \boldsymbol{\varphi}\right)_{L_{2}(\Gamma)}
$$

Let $\mathcal{E}_{\alpha}^{+}(\Gamma)$ consist of all $\boldsymbol{\Sigma} \in \mathfrak{M}^{+}(\Gamma)$ which the property

$$
\sup _{\|\boldsymbol{\varphi}\|_{H^{\beta / 2}(\Gamma)} \leq 1}\left|\left(V_{\beta} \boldsymbol{\Sigma}, \boldsymbol{\varphi}\right)_{L_{2}(\Gamma)}\right|<\infty .
$$

Hence, for $\boldsymbol{\Sigma} \in \mathcal{E}_{\alpha}^{+}(\Gamma)$, we can identify $V_{\beta} \boldsymbol{\Sigma}$ with an associated element $\boldsymbol{\psi} \in H^{-\beta / 2}(\Gamma)$ satisfying $\boldsymbol{\psi} \mathrm{d} s=\mathrm{d} V_{\beta} \boldsymbol{\Sigma}$ and

$$
\|\boldsymbol{\psi}\|_{H^{-\beta / 2}(\Gamma)}=\sup _{\|\boldsymbol{\varphi}\|_{H^{\beta / 2}(\Gamma)} \leq 1}\left|\left(V_{\beta} \boldsymbol{\Sigma}, \boldsymbol{\varphi}\right)_{L_{2}(\Gamma)}\right| .
$$


This leads us to the following theorem.

Theorem 3.3 (see also [8, Theorem 3]). For any $\boldsymbol{\Sigma} \in \mathcal{E}_{\alpha}^{+}(\Gamma)$ there exists a unique element $\boldsymbol{\sigma} \in \mathcal{K}^{\beta / 2}(\Gamma)$ such that $\mathrm{d} \boldsymbol{\Sigma}=\boldsymbol{\sigma} \mathrm{d}$ s and

$$
\boldsymbol{\Sigma}(\boldsymbol{\varphi})=\int_{\Gamma} \boldsymbol{\varphi} \mathrm{d} \boldsymbol{\Sigma}=(\boldsymbol{\varphi}, \boldsymbol{\sigma})_{L_{2}(\Gamma)} \text { for all } \boldsymbol{\varphi} \in C^{\infty}(\Gamma) .
$$

Moreover, $\mathcal{E}_{\alpha}^{+}(\Gamma)=\mathcal{K}^{\beta / 2}(\Gamma)$, and the Riesz energy $E_{\alpha}(\boldsymbol{\Sigma})$ of any $\boldsymbol{\Sigma} \in \mathcal{E}_{\alpha}^{+}(\Gamma)$ is equivalent to the $H^{\beta / 2}(\Gamma)$-norm of the corresponding $\boldsymbol{\sigma} \in \mathcal{K}^{\beta / 2}(\Gamma)$ in the sense of (3.11).

Proof. Choose an arbitrary $\boldsymbol{\Sigma} \in \mathcal{E}_{\alpha}^{+}(\Gamma)$. As has been observed just above, $V_{\beta} \boldsymbol{\Sigma} \in H^{-\beta / 2}(\Gamma)$ is a linear functional on $C^{\infty}(\Gamma)$, and it is bounded on $H^{\beta / 2}(\Gamma)$ because of

$$
\left.\left|V_{\beta} \boldsymbol{\Sigma}(\boldsymbol{\varphi})\right|=\mid\left(V_{\beta} \boldsymbol{\Sigma}, \boldsymbol{\varphi}\right)_{L_{2}(\Gamma)}\right) \mid \leq c\left\|V_{\beta} \boldsymbol{\Sigma}\right\|_{H^{-\beta / 2}(\Gamma)}\|\boldsymbol{\varphi}\|_{H^{\beta / 2}(\Gamma)} .
$$

Since $C^{\infty}(\Gamma)$ is dense in $H^{\beta / 2}(\Gamma)$, according to the Fischer-Riesz lemma on the representation of bounded linear functionals there exists a unique $\boldsymbol{\sigma}_{0} \in H^{\beta / 2}(\Gamma)$ with

$$
\int_{\Gamma} V_{\beta} \boldsymbol{\varphi} \mathrm{d} \boldsymbol{\Sigma}=\int_{\Gamma} \boldsymbol{\zeta} \mathrm{d} \boldsymbol{\Sigma}=\boldsymbol{\Sigma}(\boldsymbol{\zeta})=\int_{\Gamma} \boldsymbol{\sigma}_{0} \boldsymbol{\zeta} \mathrm{d} s=\left(\boldsymbol{\sigma}_{0}, \boldsymbol{\zeta}\right)_{L_{2}(\Gamma)},
$$

where $\boldsymbol{\zeta}:=V_{\beta} \boldsymbol{\varphi}$. If $\boldsymbol{\varphi}$ traces $C^{\infty}(\Gamma)$, so does $\boldsymbol{\zeta}$; hence, (3.12) holds for $\boldsymbol{\sigma}=\boldsymbol{\sigma}_{0} \in H^{\beta / 2}(\Gamma)$ by replacing $\boldsymbol{\zeta}$ by $\boldsymbol{\varphi}$. Since $\boldsymbol{\Sigma} \in \mathfrak{M}^{+}(\Gamma)$, we actually have $\boldsymbol{\sigma}_{0} \in \mathcal{K}^{\beta / 2}(\Gamma)$, and the inclusion $\mathcal{E}_{\alpha}^{+}(\Gamma) \subseteq \mathcal{K}^{\beta / 2}(\Gamma)$ follows.

Now, let $\boldsymbol{\sigma} \in \mathcal{K}^{\beta / 2}(\Gamma)$. Then $\boldsymbol{\psi}=V_{\beta} \boldsymbol{\sigma} \in H^{-\beta / 2}(\Gamma)$ and

$$
\begin{aligned}
\|\boldsymbol{\psi}\|_{H^{-\beta / 2}(\Gamma)} & =\left\|V_{\beta} \boldsymbol{\sigma}\right\|_{H^{-\beta / 2}(\Gamma)} \\
& =\sup _{\|\boldsymbol{\varphi}\|_{H^{\beta / 2}(\Gamma)} \leqslant 1}\left|\left(V_{\beta} \boldsymbol{\sigma}, \boldsymbol{\varphi}\right)_{L_{2}(\Gamma)}\right| \leqslant c\|\boldsymbol{\sigma}\|_{H^{\beta / 2}(\Gamma)}<\infty
\end{aligned}
$$

due to the duality $H^{-\beta / 2}(\Gamma) \times H^{\beta / 2}(\Gamma)$ of $(\cdot, \cdot)_{L_{2}(\Gamma)}, V_{\beta}$ being a pseudodifferential operator on $\Gamma$. Hence, $\mathcal{K}^{\beta / 2}(\Gamma) \subseteq \mathcal{E}_{\alpha}^{+}(\Gamma)$, which completes the proof.

Although we have shown that the distributions in $\mathcal{K}^{\beta / 2}(\Gamma)$ all have finite Riesz energy $E_{\alpha}(\boldsymbol{\mu})$, it is not clear yet whether there are no other measures in $\mathfrak{M}^{+}(\Gamma)$ whose Riesz energy is finite. To elaborate on this problem, we employ an idea by J. Deny [5]. A measure on $\Gamma$ can be considered as a distribution on $\Gamma$ and, hence, can be Fourier transformed. In connection with the localization of $V_{\beta}$ on one chart of the atlas on $\Gamma$, we have relation (3.8) where the pseudodifferential operator $V_{\ell r}$ is defined via Fourier transform.

If $\boldsymbol{\Sigma} \in \mathfrak{M}^{+}(\Gamma)$ is given, then it becomes via the pushforward $\mathcal{X}_{\ell r \star}$ the localized distribution $\boldsymbol{\Sigma}_{\ell r}:=\mathcal{X}_{\ell \star \star} \beta_{\ell r} \boldsymbol{\Sigma}$ with compact support in $\mathcal{U}_{\ell r} \subset \mathbb{R}^{n-1}$ (see e.g. [8, Lemma 5]), which can be Fourier transformed to $\widehat{\boldsymbol{\Sigma}}_{\ell r}\left(\boldsymbol{\xi}^{\prime}\right)$ on $\mathbb{R}^{n-1}$. The measures in $\mathfrak{M}^{+}(\Gamma)$ for which

$$
\int_{\mathbb{R}^{n-1}}\left|\boldsymbol{\xi}^{\prime}\right|^{\beta}\left|\widehat{\boldsymbol{\Sigma}}_{\ell r}\left(\boldsymbol{\xi}^{\prime}\right)\right|^{2} \mathrm{~d} \boldsymbol{\xi}^{\prime}<\infty
$$

are precisely all those having finite Riesz energy (cf. (3.8); observe that the first summand on the left-hand side of (3.8) is the dominant one). Let $\mathcal{E}_{\alpha}^{\star}(\Gamma)$ consist of all $\boldsymbol{\Sigma} \in \mathfrak{M}^{+}(\Gamma)$ satisfying (3.13).

Theorem 3.4. There holds

$$
\mathcal{E}_{\alpha}^{\star}(\Gamma)=\mathcal{K}^{\beta / 2}(\Gamma)
$$

Proof. Let $\boldsymbol{\Sigma}=\boldsymbol{\sigma} \in \mathcal{K}^{\beta / 2}(\Gamma)$. Then, by Theorem 3.2,

$$
\left(V_{\beta} \boldsymbol{\sigma}, \boldsymbol{\sigma}\right)_{L_{2}(\Gamma)}=E_{\alpha}(\boldsymbol{\sigma})<\infty .
$$


With (3.8) and Parseval's identity, together with $\boldsymbol{\Sigma}_{\ell r} \in H^{\beta / 2}\left(\mathcal{U}_{\ell r}\right)$, we obtain further

$$
\begin{aligned}
& \int_{\mathbb{R}^{n-1}}\left|\boldsymbol{\xi}^{\prime}\right|^{\beta}\left|\widehat{\boldsymbol{\Sigma}}_{\ell r}\left(\boldsymbol{\xi}^{\prime}\right)\right|^{2} \mathrm{~d} \boldsymbol{\xi}^{\prime} \\
& \quad \leq\left|\left(V_{\ell r} \boldsymbol{\Sigma}_{\ell r}, \boldsymbol{\Sigma}_{\ell r}\right)_{L^{2}\left(\mathcal{U}_{\ell r}\right)}\right|+\left|\left(A_{\ell r}^{\beta-2} \boldsymbol{\Sigma}_{\ell r}, \boldsymbol{\Sigma}_{\ell r}\right)_{L^{2}\left(\mathcal{U}_{\ell r}\right)}\right|+\left|\left(R_{\ell r} \boldsymbol{\Sigma}_{\ell r}, \boldsymbol{\Sigma}_{\ell r}\right)_{L^{2}\left(\mathcal{U}_{\ell r}\right)}\right| \\
& \quad \leq c\left\|\boldsymbol{\Sigma}_{\ell r}\right\|_{H^{\beta / 2}\left(\mathcal{U}_{\ell r}\right)}^{2}+c^{\prime}\left\|\boldsymbol{\Sigma}_{\ell r}\right\|_{H^{\beta / 2+2-\beta}\left(\mathcal{U}_{\ell r}\right)}|| \boldsymbol{\Sigma}_{\ell r} \|_{H^{\beta / 2}\left(\mathcal{U}_{\ell r}\right)} \\
& \quad \leq c^{\prime \prime}\left\|\boldsymbol{\Sigma}_{\ell r}\right\|_{H^{\beta / 2}\left(\mathcal{U}_{\ell r}\right)}^{2}<\infty
\end{aligned}
$$

since $2-\beta / 2 \geq \beta / 2$. Consequently, it holds $\boldsymbol{\sigma}=\boldsymbol{\Sigma} \in \mathcal{E}_{\alpha}^{\star}(\Gamma)$ and thus $\mathcal{K}^{\beta / 2}(\Gamma) \subseteq \mathcal{E}_{\alpha}^{\star}(\Gamma)$.

Next, suppose $\boldsymbol{\Sigma} \in \mathcal{E}_{\alpha}^{\star}(\Gamma)$; then by localization inequality (3.13) holds. Since $\left|\boldsymbol{\xi}^{\prime}\right|^{\beta} \mid(1+$ $\left.\left|\boldsymbol{\xi}^{\prime}\right|^{2}\right)^{-\beta / 2} \leq 1$ we find

$$
\begin{aligned}
\left\|V_{\ell r} \boldsymbol{\Sigma}_{\ell r}\right\|_{H^{-\beta / 2}\left(\mathcal{U}_{\ell r}\right)}^{2} & =\int_{\mathbb{R}^{n-1}}\left|\boldsymbol{\xi}^{\prime}\right|^{2 \beta}\left(1+\left|\boldsymbol{\xi}^{\prime}\right|^{2}\right)^{-\beta / 2}\left|\widehat{\boldsymbol{\Sigma}}_{\ell r}\left(\boldsymbol{\xi}^{\prime}\right)\right|^{2} \mathrm{~d} \boldsymbol{\xi}^{\prime} \\
& \leq \int_{\mathbb{R}^{n-1}}\left|\boldsymbol{\xi}^{\prime}\right|^{\beta}\left|\widehat{\boldsymbol{\Sigma}}_{\ell r}\left(\boldsymbol{\xi}^{\prime}\right)\right|^{2} \mathrm{~d} \boldsymbol{\xi}^{\prime}<\infty
\end{aligned}
$$

With pullback to $\Gamma$ this implies that

$$
\left\|V_{\beta} \boldsymbol{\Sigma}\right\|_{H^{-\beta / 2}(\Gamma)}<\infty
$$

Application of Theorem 3.3 then gives $\boldsymbol{\Sigma} \in \mathcal{E}_{\alpha}^{+}(\Gamma)=\mathcal{K}^{\beta / 2}(\Gamma)$, which in view of the arbitrary choice of $\boldsymbol{\Sigma} \in \mathcal{E}_{\alpha}^{\star}(\Gamma)$ finally yields $\mathcal{K}^{\beta / 2}(\Gamma)=\mathcal{E}_{\alpha}^{\star}(\Gamma)$.

Theorem 3.5 (see also [8, Theorem 4]). Let $\boldsymbol{\Sigma} \in \mathfrak{M}^{+}(\Gamma)$ with $E_{\alpha}(\boldsymbol{\Sigma})<\infty$. Then there exists a sequence of absolutely continuous measures $\boldsymbol{\Sigma}_{k} \in \mathcal{K}^{\beta / 2}(\Gamma)$, where $\mathrm{d} \boldsymbol{\Sigma}_{k}=\sum_{i \in I} \alpha_{i} \varphi_{k}^{i} \mathrm{~d}$ s with $\varphi_{k}^{i} \in C\left(\Gamma_{i}\right) \cap H^{\beta / 2}\left(\Gamma_{i}\right)$ and $\varphi_{k}^{i}(\mathbf{x}) \geqslant 0$ for $\mathbf{x} \in \Gamma_{i}$, such that $\left\{\boldsymbol{\Sigma}_{k}\right\}_{k \in \mathbb{N}}$ converges weakly and strongly in the Hilbert space $H^{\beta / 2}(\Gamma)$ to $\boldsymbol{\Sigma}$, i.e.,

$$
\boldsymbol{\Sigma}_{k}(\boldsymbol{\varphi}) \stackrel{k \rightarrow \infty}{\longrightarrow} \boldsymbol{\Sigma}(\boldsymbol{\varphi}) \text { for all } \boldsymbol{\varphi} \in C(\Gamma) \text { and } \lim _{k \rightarrow \infty}\left\|\boldsymbol{\Sigma}-\boldsymbol{\Sigma}_{k}\right\|_{H^{\beta / 2}(\Gamma)}=0 .
$$

Proof. For $\boldsymbol{\Sigma}$ we have $\boldsymbol{\Sigma}=\boldsymbol{\sigma} \in \mathcal{K}^{\beta / 2}(\Gamma)$ due to Theorem 3.3. Hence, since $C^{\infty}(\Gamma) \subset H^{\beta / 2}(\Gamma)$ densely, there exists a sequence $\boldsymbol{\sigma}_{k}=\sum_{i \in I} \alpha_{i} \sigma_{k}^{i} \in C^{\infty}(\Gamma)$ with $\left\|\boldsymbol{\sigma}-\boldsymbol{\sigma}_{k}\right\|_{H^{\beta / 2}(\Gamma)}<\frac{1}{k}$ for all $k \in \mathbb{N}$. We define $\boldsymbol{\Sigma}_{k} \in \mathfrak{M}^{+}(\Gamma)$ by

$$
\mathrm{d} \boldsymbol{\Sigma}_{k}=\overline{\boldsymbol{\sigma}}_{k} \mathrm{~d} s \quad \text { with } \quad \overline{\boldsymbol{\sigma}}_{k}=\sum_{i \in I} \alpha_{i} \bar{\sigma}_{k}^{i}
$$

where $\bar{\sigma}_{k}^{i}(\mathbf{x}):=\max \left\{0, \sigma_{k}^{i}(\mathbf{x})\right\}$. Then, $\bar{\sigma}_{k}^{i} \in C\left(\Gamma_{i}\right) \cap H^{\beta / 2}\left(\Gamma_{i}\right)$ since $\bar{\sigma}_{k}^{i}$ is piecewise smooth and

$$
\begin{aligned}
\left\|\boldsymbol{\sigma}-\overline{\boldsymbol{\sigma}}_{k}\right\|_{H^{\beta / 2}(\Gamma)} & =\left\|\boldsymbol{\sigma}-\boldsymbol{\sigma}_{k}+\boldsymbol{\sigma}_{k}-\overline{\boldsymbol{\sigma}}_{k}\right\|_{H^{\beta / 2}(\Gamma)} \\
& \leq\left\|\boldsymbol{\sigma}-\boldsymbol{\sigma}_{k}\right\|_{H^{\beta / 2}(\Gamma)}+\left\|\boldsymbol{\sigma}_{k}-\overline{\boldsymbol{\sigma}}_{k}\right\|_{H^{\beta / 2}(\Gamma)} \\
& =\left\|\boldsymbol{\sigma}-\boldsymbol{\sigma}_{k}\right\|_{H^{\beta / 2}(\Gamma)}+\sum_{i \in I}\left\|\underline{\sigma}_{k}^{i}\right\|_{H^{\beta / 2}\left(\Gamma_{i}\right)} .
\end{aligned}
$$

Herein, it holds $\underline{\sigma}_{k}^{i}(\mathbf{x})=\min \left\{0, \sigma_{k}^{i}(\mathbf{x})\right\} \leq 0$ for all $\mathbf{x} \in \Gamma_{i}$, particularly $\underline{\sigma}_{k}^{i} \in C\left(\Gamma_{i}\right) \cap H^{\beta / 2}\left(\Gamma_{i}\right)$. From $\sigma^{i} \geq 0$, it immediately follows that

$$
\left\|\underline{\sigma}_{k}^{i}\right\|_{H^{\beta / 2}\left(\Gamma_{i}\right)} \leq\left\|\sigma^{i}-\sigma_{k}^{i}\right\|_{H^{\beta / 2}\left(\Gamma_{i}\right)}
$$

for all $i \in I$ and therefore

$$
\left\|\boldsymbol{\sigma}-\overline{\boldsymbol{\sigma}}_{k}\right\|_{H^{\beta / 2}(\Gamma)} \leq 2\left\|\boldsymbol{\sigma}-\boldsymbol{\sigma}_{k}\right\|_{H^{\beta / 2}(\Gamma)} \leq \frac{2}{k} \stackrel{k \rightarrow \infty}{\longrightarrow} 0
$$

as desired. 


\section{The Gauss problem}

The Gauss variational problem is the problem of minimizing the Riesz energy for particularly signed Borel measures on the given $(n-1)$-dimensional manifold $\Gamma \subset \mathbb{R}^{n}$, in the presence of an external field. Let $\mathbf{g}$ be a given continuous, positive function on $\Gamma$ and $\mathbf{a}=\left(a_{i}\right)_{i \in I}$ a given vector with $a_{i}>0, i \in I$. Then, the set of admissible charges for the Gauss problem is defined as

$$
\mathcal{E}_{\alpha}(\Gamma, \mathbf{a}, \mathbf{g}):=\left\{\boldsymbol{\mu} \in \mathcal{K}^{\beta / 2}(\Gamma): \int_{\Gamma_{i}} g_{i} \mathrm{~d} \mu^{i}=a_{i} \text { for all } i \in I\right\}
$$

where we set $g_{i}:=\mathbf{g}_{\mid \Gamma_{i}}$. Note that the set $\mathcal{E}_{\alpha}(\Gamma, \mathbf{a}, \mathbf{g})$ is convex and weakly and strongly closed in $\mathcal{K}^{\beta / 2}(\Gamma)$

The Gauss minimal energy problem reads as follows (see [19] and [15]): To given $\mathbf{a} \in \mathbb{R}_{+}^{|I|}$, $\mathbf{f} \in C(\Gamma)$ and $\mathbf{g} \in C(\Gamma)$ such that $\mathbf{g}>\mathbf{0}$, find the Borel measure $\boldsymbol{\mu}_{0} \in \mathcal{E}_{\alpha}(\Gamma, \mathbf{a}, \mathbf{g})$ which is the minimizer of

$$
\inf _{\boldsymbol{\mu} \in \mathcal{E}_{\alpha}(\Gamma, \mathbf{a}, \mathbf{g})} \mathbb{G}_{\mathbf{f}}(\boldsymbol{\mu})=\mathbb{G}_{\mathbf{f}}\left(\boldsymbol{\mu}_{0}\right)=: \mathbb{G}_{\mathbf{f}}(\Gamma, \mathbf{a}, \mathbf{g})
$$

where the Gauss functional is given by

$$
\mathbb{G}_{\mathbf{f}}(\boldsymbol{\mu}):=E_{\alpha}(\boldsymbol{\mu})-2 \int_{\Gamma} \mathbf{f} \mathrm{d} \boldsymbol{\mu} .
$$

Since $\mathbb{G}_{\mathbf{f}}(\boldsymbol{\mu})$ is on $\mathcal{E}_{\alpha}(\Gamma, \mathbf{a}, \mathbf{g})$ strictly convex and weakly and strongly continuous, the Gauss problem admits a unique solution $\boldsymbol{\mu}_{0} \in \mathcal{E}_{\alpha}(\Gamma, \mathbf{a}, \mathbf{g})$.

Based on Theorem 3.2, the minimization problem (4.1) can also be formulated as a variational problem in $H^{\beta / 2}(\Gamma)$. Namely, minimize the functional

$$
\mathbb{V}_{\mathbf{f}}(\boldsymbol{\varphi}):=\left(\boldsymbol{\varphi}, V_{\beta} \boldsymbol{\varphi}\right)_{L_{2}(\Gamma)}-2(\mathbf{f}, \boldsymbol{\varphi})_{L_{2}(\Gamma)}, \quad \boldsymbol{\varphi} \in H^{\beta / 2}(\Gamma),
$$

over the affine cone

$$
\begin{aligned}
\mathcal{K}(\Gamma, \mathbf{a}, \mathbf{g}):= & \left\{\varphi=\sum_{i \in I} \alpha_{i} \varphi^{i} \text { where } \varphi^{i} \in H^{\beta / 2}\left(\Gamma_{i}\right),\right. \\
& \left.\varphi^{i} \geq 0 \text { and } \int_{\Gamma_{i}} g_{i} \varphi^{i} \mathrm{~d} s=a_{i}>0 \text { for all } i \in I\right\} \subset \mathcal{K}^{\beta / 2}(\Gamma) \subset H^{\beta / 2}(\Gamma)
\end{aligned}
$$

where $\mathbf{f} \in C(\Gamma), \mathbf{g}>0, \mathbf{g} \in C(\Gamma)$ and $\mathbf{a} \in \mathbb{R}_{+}^{|I|}$ are given. This minimization problem will be called the dual Gauss problem.

Theorem 4.1. To the unique solution $\boldsymbol{\mu}_{0} \in \mathcal{E}_{\alpha}(\Gamma, \mathbf{a}, \mathbf{g})$ of the Gauss problem (4.1), there corresponds a unique element $\varphi_{0} \in \mathcal{K}(\Gamma, \mathbf{a}, \mathbf{g}) \subset H^{\beta / 2}(\Gamma)$ with the properties

$$
\boldsymbol{\mu}_{0}(\boldsymbol{\varphi})=\left(\boldsymbol{\varphi}_{0}, \boldsymbol{\varphi}\right)_{L_{2}(\Gamma)} \text { for all } \varphi \in C^{\infty}(\Gamma)
$$

and

$$
\mathbb{V}_{\mathbf{f}}\left(\boldsymbol{\varphi}_{0}\right)=\mathbb{G}_{\mathbf{f}}\left(\boldsymbol{\mu}_{0}\right)=\mathbb{G}_{\mathbf{f}}(\Gamma, \mathbf{a}, \mathbf{g}) .
$$

The element $\varphi_{0}$ is the minimizer of the functional $\mathbb{V}_{\mathbf{f}}$ over $\mathcal{K}(\Gamma, \mathbf{a}, \mathbf{g})$, i.e.,

$$
\mathbb{V}_{\mathbf{f}}\left(\boldsymbol{\varphi}_{0}\right)=\min _{\boldsymbol{\varphi} \in \mathcal{K}(\Gamma, \mathbf{a}, \mathbf{g})} \mathbb{V}_{\mathbf{f}}(\boldsymbol{\varphi})=: \mathbb{V}_{\mathbf{f}}(\Gamma, \mathbf{a}, \mathbf{g})
$$

Proof. By Theorems 3.2 and 3.3, to any Borel measure $\boldsymbol{\mu}=\sum_{i \in I} \alpha_{i} \mu^{i} \in \mathcal{E}_{\alpha}(\Gamma, \mathbf{a}, \mathbf{g})$, there corresponds a unique element $\boldsymbol{\sigma}_{\mu}=\sum_{i \in I} \alpha_{i} \sigma_{\mu}^{i} \in \mathcal{K}^{\beta / 2}(\Gamma)$ satisfying both (3.11) and (3.12). Moreover, since $C^{\infty}(\Gamma)$ is dense in $C(\Gamma)$, from $(3.12)$ we get

$$
\left(\sigma_{\mu}^{i}, g_{i}\right)_{L_{2}\left(\Gamma_{i}\right)}=\mu^{i}\left(g_{i}\right)=a_{i} \text { for all } i \in I .
$$

Hence, $\boldsymbol{\sigma}_{\mu} \in \mathcal{K}(\Gamma, \mathbf{a}, \mathbf{g})$.

Applying (3.11), for these $\boldsymbol{\mu}$ and $\boldsymbol{\sigma}_{\mu}$ we also obtain

$$
\mathbb{V}_{\mathbf{f}}\left(\boldsymbol{\sigma}_{\mu}\right)=\left(V_{\beta} \boldsymbol{\sigma}_{\mu}, \boldsymbol{\sigma}_{\mu}\right)_{L^{2}(\Gamma)}-2\left(\boldsymbol{\sigma}_{\mu}, \mathbf{f}\right)_{L^{2}(\Gamma)}=E_{\alpha}(\boldsymbol{\mu})-2 \boldsymbol{\mu}(\mathbf{f})=\mathbb{G}_{\mathbf{f}}(\boldsymbol{\mu}) .
$$


Thus, the correspondence $\boldsymbol{\mu} \mapsto \boldsymbol{\sigma}_{\mu}$ between $\mathcal{E}_{\alpha}(\Gamma, \mathbf{a}, \mathbf{g})$ and $\mathcal{K}(\Gamma, \mathbf{a}, \mathbf{g})$ is one-to-one and satisfies (4.4), which immediately implies

$$
\mathbb{G}_{\mathbf{f}}(\Gamma, \mathbf{a}, \mathbf{g})=\mathbb{V}_{\mathbf{f}}(\Gamma, \mathbf{a}, \mathbf{g}) .
$$

If now $\boldsymbol{\mu}_{0}$ is the (unique) solution of the Gauss problem (4.1), then $\boldsymbol{\varphi}_{0}$, the image of $\boldsymbol{\mu}_{0}$ under this correspondence, is the unique solution of the minimizing problem (4.3), and vice versa.

\section{The PARTICULAR CASE $\alpha=\mathbf{0}$}

In the following, we will focus on the particular situation $\alpha=0$ from the potential theoretic point of view. The double layer energy $E_{0}$ of a function $\varphi \in H^{\beta / 2}(\Gamma), \beta=1-\alpha=1$, which is harmonic in $\Omega$ (see [12, Equation (1.2.17)]) is given by

$$
E_{0}(\varphi):=(\mathbf{D} \varphi, \varphi)_{L_{2}(\Gamma)}
$$

with the hypersingular integral operator $\mathbf{D}$ :

$$
\begin{gathered}
\mathbf{D} \varphi(\mathbf{x}):=\text { p.f. } \int_{\Gamma \backslash\{\mathbf{x}\}} k_{\mathbf{D}}(\mathbf{x}, \mathbf{y}) \boldsymbol{\varphi}(\mathbf{y}) \mathrm{d} s_{\mathbf{y}} \\
k_{\mathbf{D}}(\mathbf{x}, \mathbf{y})=k_{\mathbf{D}}(\mathbf{y}, \mathbf{x})=c_{n}\left\{\frac{\mathbf{n}(\mathbf{x}) \cdot \mathbf{n}(\mathbf{y})}{|\mathbf{x}-\mathbf{y}|^{n}}+n \frac{(\mathbf{y}-\mathbf{x}) \cdot \mathbf{n}(\mathbf{y})(\mathbf{x}-\mathbf{y}) \cdot \mathbf{n}(\mathbf{x})}{|\mathbf{x}-\mathbf{y}|^{n+2}}\right\} .
\end{gathered}
$$

The Hadamard partie finie integral operator is given by the finite part with respect to $0<\delta \rightarrow 0$ of

$$
\begin{aligned}
& \quad \int_{\mathbf{y} \in \Gamma \wedge|\mathbf{x}-\mathbf{y}|>\delta} k_{\mathbf{D}}(\mathbf{x}, \mathbf{y}) \boldsymbol{\varphi}(\mathbf{y}) \mathrm{d} s_{\mathbf{y}} \\
& \quad=\int_{\mathbf{y} \in \Gamma \wedge|\mathbf{x}-\mathbf{y}|>\delta} k_{\mathbf{D}}(\mathbf{x}, \mathbf{y})\{\boldsymbol{\varphi}(\mathbf{y})-\boldsymbol{\varphi}(\mathbf{x})\} \mathrm{d} s_{\mathbf{y}}+\int_{\mathbf{y} \in \Gamma \wedge|\mathbf{x}-\mathbf{y}|>\delta} k_{\mathbf{D}}(\mathbf{x}, \mathbf{y}) \mathrm{d} s_{\mathbf{y}} \boldsymbol{\varphi}(\mathbf{x})
\end{aligned}
$$

if $\varphi \in C^{\infty}(\Gamma)$. The limit

$$
\lim _{\delta \rightarrow 0} \int_{0<\delta<|\mathbf{x}-\mathbf{y}|} k_{\mathbf{D}}(\mathbf{x}, \mathbf{y})\{\boldsymbol{\varphi}(\mathbf{y})-\boldsymbol{\varphi}(\mathbf{x})\} \mathrm{d} s_{\mathbf{y}}=\text { p.v. } \int_{\mathbf{y} \in \Gamma \backslash\{\mathbf{x}\}} k_{\mathbf{D}}(\mathbf{x}, \mathbf{y})\{\boldsymbol{\varphi}(\mathbf{y})-\boldsymbol{\varphi}(\mathbf{x})\} \mathrm{d} s_{\mathbf{y}}
$$

exists (as a Cauchy principal value integral), whereas, from $\int_{|\mathbf{x}-\mathbf{y}|>\delta} k_{\mathbf{D}}(\mathbf{x}, \mathbf{y}) \mathrm{d} s_{\mathbf{y}}$, we have to take the finite part

$$
\lim _{\delta \rightarrow 0} \text { p.f. } \int_{|\mathbf{x}-\mathbf{y}|>\delta} k_{\mathbf{D}}(\mathbf{x}, \mathbf{y}) \mathrm{d} s_{\mathbf{y}}=: h(\mathbf{x}) .
$$

(For the evaluation of $h(\mathbf{x})$, see (A.1).) Hence, we finally arrive at

$$
\mathbf{D} \varphi(\mathbf{x})=\text { p.v. } \int_{\Gamma \backslash\{\mathbf{x}\}} k_{\mathbf{D}}(\mathbf{x}, \mathbf{y})\{\boldsymbol{\varphi}(\mathbf{y})-\boldsymbol{\varphi}(\mathbf{x})\} \mathrm{d} s_{\mathbf{y}}+h(\mathbf{x}) \boldsymbol{\varphi}(\mathbf{x}) .
$$

\section{A perturbed minimal Riesz energy problem}

Instead of considering the continuous minimal Riesz energy problem, D.P. Hardin and E.B. Saff investigate in [11] the discrete Riesz energy problem of minimizing $\stackrel{\circ}{E}_{s}\left(\boldsymbol{\omega}_{N}\right), s>d$, of the sum of a finite number $N$ of Dirac measures $\delta_{\mathbf{x}_{i}, N}, \boldsymbol{\omega}_{N}:=\left\{\mathbf{x}_{1}, \ldots, \mathbf{x}_{N}\right\}$ being a set on a $d$-rectifiable manifold $A$. The energy $\stackrel{\circ}{E}_{s}\left(\boldsymbol{\omega}_{N}\right)$ is defined by removing the self-interactions. For the sake of simplicity, assume that $A$ is compact and has the positive Hausdorff measure $\mathcal{H}_{d}(A)>0$. Then, the infimum of $\stackrel{\circ}{E}_{s}\left(\boldsymbol{\omega}_{N}\right)$ over all point sets $\boldsymbol{\omega}_{N} \subset A$ is attained at some $\boldsymbol{\omega}_{N}^{\star}:=\left\{\mathbf{x}_{1}^{\star}, \ldots, \mathbf{x}_{N}^{\star}\right\}$. In particular, it is shown in [11, Theorem 2.4] that

$$
\lim _{N \rightarrow \infty} \stackrel{\circ}{E}_{s}\left(\boldsymbol{\omega}_{N}^{\star}\right) N^{-(1+s / d)}=C_{s d} / \mathcal{H}_{d}(A)^{s / d}
$$


where $C_{s d}$ is a constant independent of $A$ and defined explicitly by the unit cube. Furthermore, in the weak-star topology of measures we have

$$
\frac{1}{N} \sum_{i=1}^{N} \delta_{\mathbf{x}_{i}, N} \rightarrow \frac{\left.\mathcal{H}_{d}(\cdot)\right|_{A}}{\mathcal{H}_{d}(A)} \quad \text { as } N \rightarrow \infty
$$

If $A$ is a bi-Lipschitz image of a single compact set in $\mathbb{R}^{d}$, then the separation estimate [11, Eq. (16)] holds for an optimal $N$ point s-energy configuration $\boldsymbol{\omega}_{N}^{\star}$ for $A$.

Now, for our $(n-1)$-dimensional manifold $\Gamma=\bigcup_{i \in I} \Gamma_{i}$, every compact smooth $\Gamma_{i}$ immersed into $\mathbb{R}^{n}$, satisfies all the assumptions on $A$ with $s=n-\alpha,-1<\alpha<1, d^{\prime}=n>d=n-1$. Following the inspiration of these results, in our continuous setting, this corresponds to integrating for a small $\delta>0$ over $(\Gamma \times \Gamma) \backslash\{|\mathbf{x}-\mathbf{y}| \leq \delta\}$, i.e., by cutting out a set with $|\mathbf{x}-\mathbf{y}| \leq \delta$ near the singularity. In order to explain the computations in the next section, we shall focus first on the following perturbation problem which, for $0<\varepsilon=\varepsilon(\delta) \rightarrow 0$, is essentially the minimization problem we will finally get.

Theorem 6.1. For $\varepsilon>0$ sufficiently small, consider the minimization problem:

$$
\left(V_{\beta} \boldsymbol{\sigma}, \boldsymbol{\sigma}\right)_{L_{2}(\Gamma)}+\frac{1}{\varepsilon}(\boldsymbol{\sigma}, \boldsymbol{\sigma})_{L_{2}(\Gamma)}-2(\mathbf{f}, \boldsymbol{\sigma})_{L_{2}(\Gamma)} \rightarrow \min
$$

subject to

$$
\int_{\Gamma_{i}} g_{i} \sigma^{i} \mathrm{~d} s=a^{i}, j \in I .
$$

Let the given data satisfy the additional conditions:

$$
\mathbf{f} \in H^{\beta / 2}(\Gamma), \mathbf{g} \in H^{\frac{3}{2} \beta}(\Gamma) \text { and } 0<a^{i} \in \mathbb{R}, i \in I .
$$

Then, the minimizer $\boldsymbol{\sigma}_{\varepsilon}^{\star} \in L_{2}(\Gamma)$ admits the asymptotic expansion

$$
\boldsymbol{\sigma}_{\varepsilon}^{\star}=\boldsymbol{\sigma}_{0}+\varepsilon \boldsymbol{\sigma}_{1}+\varepsilon^{2} \boldsymbol{\sigma}_{2} \text { satisfying }\left\|\boldsymbol{\sigma}_{j}\right\|_{\mathcal{H}_{\varepsilon}} \leq c, j=0,1,2
$$

with a constant $c>0$ independent of $\varepsilon$, and where

$$
\mathcal{H}_{\varepsilon}:=\left\{\boldsymbol{\varphi} \in H^{\beta / 2}(\Gamma) \text { with }\|\varphi\|_{\mathcal{H}_{\varepsilon}}^{2}:=\left(\varepsilon V_{\beta} \varphi, \varphi\right)_{L_{2}(\Gamma)}+\|\varphi\|_{L_{2}(\Gamma)}^{2}\right\} \subset H^{\beta / 2}(\Gamma) .
$$

In particular, with $f_{k}:=\mathbf{f}_{\mid \Gamma_{k}}$, it holds

$$
\begin{aligned}
& \boldsymbol{\sigma}_{0}=\left(\alpha_{k} \sigma_{0}^{k}\right)_{k \in I}=\left(\alpha_{k} g_{k} a^{k}\left(g_{k}, g_{k}\right)_{L_{2}\left(\Gamma_{k}\right)}^{-1}\right)_{k \in I}, \\
& \boldsymbol{\sigma}_{1}=\left(\alpha_{k} \sigma_{1}^{k}\right)_{k \in I}=\left(\alpha_{k} g_{k}\left(g_{k}, g_{k}\right)_{L_{2}\left(\Gamma_{k}\right)}^{-1}\left(g_{k}, V_{\beta} \boldsymbol{\sigma}_{0}-f_{k}\right)_{L_{2}\left(\Gamma_{k}\right)}\right)_{k \in I}-V_{\beta} \boldsymbol{\sigma}_{0}+\mathbf{f} .
\end{aligned}
$$

Proof. The quadratic form in (6.1) induces for $\varepsilon>0$ the $\varepsilon$-dependent family of Hilbert spaces $\mathcal{H}_{\varepsilon}$. Let us denote by $\mathcal{H}_{\varepsilon}^{\prime}$ the dual space to $\mathcal{H}_{\varepsilon}$ whose norm is then defined by

$$
\|\mathbf{f}\|_{\mathcal{H}_{\varepsilon}^{\prime}}:=\sup _{\mathbf{w} \in H^{\beta / 2}(\Gamma) \backslash\{0\}} \frac{(\mathbf{f}, \mathbf{w})_{L_{2}(\Gamma)}}{\|\mathbf{w}\|_{\mathcal{H}_{\varepsilon}}},
$$

satisfying the estimate

$$
\|\mathbf{f}\|_{\mathcal{H}_{\varepsilon}^{\prime}} \leq \sup _{\mathbf{w} \in H^{\beta / 2}(\Gamma) \backslash\{0\}} \frac{(\mathbf{f}, \mathbf{w})_{L_{2}(\Gamma)}}{\|\mathbf{w}\|_{L_{2}(\Gamma)}}=\|\mathbf{f}\|_{L_{2}(\Gamma)}
$$

since $H^{\beta / 2}(\Gamma) \hookrightarrow L_{2}(\Gamma)$ densely and the unit ball in $\mathcal{H}_{\varepsilon}$ is contained in the ball $\|\mathbf{w}\|_{L_{2}(\Gamma)} \leq 1$.

The problem (6.1) can also be written as to minimize

$$
\mathbf{J}_{\varepsilon}(\boldsymbol{\sigma}):=\frac{1}{2}\|\boldsymbol{\sigma}\|_{\mathcal{H}_{\varepsilon}}^{2}-\varepsilon(\mathbf{f}, \boldsymbol{\sigma})_{L_{2}(\Gamma)}
$$

subject to (6.2). It possesses the Lagrangian

$$
\mathbf{L}_{\lambda}(\boldsymbol{\sigma}):=\frac{1}{2}\|\boldsymbol{\sigma}\|_{\mathcal{H}_{\varepsilon}}^{2}-\varepsilon(\mathbf{f}, \boldsymbol{\sigma})_{L_{2}(\Gamma)}+\sum_{j \in I} \alpha_{j} \lambda_{j}\left(a^{j}-\left(g_{j}, \sigma_{j}\right)_{L_{2}\left(\Gamma_{j}\right)}\right),
$$


where $\boldsymbol{\sigma}=\sum_{j \in I} \alpha_{j} \sigma^{j}$ and $\alpha_{j} \sigma^{j} \mathrm{~d} s=\boldsymbol{\Sigma}_{\mid \Gamma_{j}}$. Thus, the necessary conditions at the minimum read as

$$
\partial_{\sigma^{k}} \mathbf{L}_{\lambda}\left(\boldsymbol{\sigma}_{\varepsilon}\right)=\varepsilon \alpha_{k} V_{\beta} \sigma_{\varepsilon}^{k}+\alpha_{k} \sigma_{\varepsilon}^{k}-\varepsilon \alpha_{k} f_{k}-\alpha_{k} \lambda_{k} g_{k}=0
$$

or

$$
\varepsilon V_{\beta} \sigma_{\varepsilon}^{k}+\sigma_{\varepsilon}^{k}-\varepsilon f_{k}=\lambda_{k} g_{k} \text { and }\left(g_{k}, \sigma_{\varepsilon}^{k}\right)_{L_{2}\left(\Gamma_{k}\right)}=a^{k}, k \in I .
$$

Here, $\boldsymbol{\lambda} \neq \mathbf{0}$ since the constraints (6.3) are always active as it follows from (6.5) and will also be seen below.

For $\boldsymbol{\sigma}$ and $\boldsymbol{\lambda}$, we insert the expansion (6.4) into (6.6) and obtain the system

$$
\begin{gathered}
\varepsilon V_{\beta} \sigma_{0}^{k}+\sigma_{0}^{k}-\varepsilon f_{k}+\varepsilon^{2} V_{\beta} \sigma_{1}^{k}+\varepsilon \sigma_{1}^{k}+\varepsilon^{3} V_{\beta} \sigma_{2}^{k}+\varepsilon^{2} \sigma_{2}^{k}=\left(\lambda_{0}^{k}+\varepsilon \lambda_{1}^{k}+\varepsilon^{2} \lambda_{2}^{k}\right) g_{k} \text { on } \Gamma_{k}, \\
\left(g_{k}, \sigma_{0}^{k}\right)_{L_{2}\left(\Gamma_{k}\right)}+\varepsilon\left(g_{k}, \sigma_{1}^{k}\right)_{L_{2}\left(\Gamma_{k}\right)}+\varepsilon^{2}\left(g_{k}, \sigma_{2}^{k}\right)_{L_{2}\left(\Gamma_{k}\right)}=a^{k}, k \in I .
\end{gathered}
$$

Equating equal order terms in $\varepsilon$ yields with $\lambda_{0}^{j}>0$ :

Order $\varepsilon^{0}$ : We find

$$
\sigma_{0}^{j}=g_{j} \lambda_{0}^{j} \text { and }\left(g_{j}, \sigma_{0}^{j}\right)_{L_{2}\left(\Gamma_{j}\right)}=\left(g_{j}, g_{j}\right)_{L_{2}\left(\Gamma_{j}\right)} \lambda_{0}^{j}=a^{j}>0 .
$$

Thus, it follows

$$
\lambda_{0}^{j}=\left(g_{j}, g_{j}\right)_{L_{2}\left(\Gamma_{j}\right)}^{-1} a^{j} \text { and } \sigma_{0}^{k}=g_{k} a^{k}\left(g_{k}, g_{k}\right)_{L_{2}\left(\Gamma_{k}\right)}^{-1} \in H^{\frac{3}{2} \beta}\left(\Gamma_{k}\right) .
$$

The assumptions (6.3) imply the properties $\sigma_{0}^{k}>0$. Moreover,

$$
\left\|\sigma_{0}^{k}\right\|_{\mathcal{H}_{\varepsilon}} \leq c_{1}\left\|\sigma_{0}^{k}\right\|_{H^{\frac{3}{2} \beta}\left(\Gamma_{k}\right)} \leq c .
$$

Order $\varepsilon^{1}$ : It holds

$$
-f_{k}+\sigma_{1}^{k}=\lambda_{1}^{k} g_{k}-V_{\beta} \sigma_{0}^{k}
$$

and

$$
\left(V_{\beta} \sigma_{0}^{k}, g_{k}\right)_{L_{2}\left(\Gamma_{k}\right)}-\left(f_{k}, g_{k}\right)_{L_{2}\left(\Gamma_{k}\right)}+\left(\sigma_{1}^{k}, g_{k}\right)_{L_{2}\left(\Gamma_{k}\right)}=\lambda_{1}^{k}\left(g_{k}, g_{k}\right)_{L_{2}\left(\Gamma_{k}\right)} .
$$

This yields with $\left(\sigma_{1}^{k}, g_{k}\right)_{L_{2}\left(\Gamma_{k}\right)}=0$ :

$$
\begin{gathered}
\lambda_{1}^{k}=\left(g_{k}, g_{k}\right)_{L_{2}\left(\Gamma_{k}\right)}^{-1}\left(V_{\beta} \sigma_{0}^{k}-f_{k}, g_{k}\right)_{L_{2}\left(\Gamma_{k}\right)}, \\
\sigma_{1}^{k}=g_{k}\left(g_{k}, g_{k}\right)_{L_{2}\left(\Gamma_{k}\right)}^{-1}\left(V_{\beta} \sigma_{0}^{k}-f_{k}, g_{k}\right)_{L_{2}\left(\Gamma_{k}\right)}+f_{k}-V_{\beta} \sigma_{0}^{k} \in H^{\beta / 2}\left(\Gamma_{k}\right) .
\end{gathered}
$$

Hence,

$$
\left\|\sigma_{1}^{k}\right\|_{\mathcal{H}_{\varepsilon}} \leq c_{2}\left\|\sigma_{0}^{k}\right\|_{H^{\beta / 2}\left(\Gamma_{k}\right)} \leq c
$$

Order $\varepsilon^{2}$ : We derive the identities

$$
\begin{gathered}
V_{\beta} \sigma_{1}^{k}+\left(\varepsilon V_{\beta} \sigma_{2}^{k}+\sigma_{2}^{k}\right)=\lambda_{2}^{k} g_{k}, \\
\varepsilon\left(V_{\beta} \sigma_{2}^{k}, g_{k}\right)_{L_{2}\left(\Gamma_{k}\right)}+\left(V_{\beta} \sigma_{1}^{k}, g_{k}\right)_{L_{2}\left(\Gamma_{k}\right)}=\lambda_{2}^{k}\left(g_{k}, g_{k}\right)_{L_{2}\left(\Gamma_{k}\right)}
\end{gathered}
$$

since $\left(\sigma_{2}^{k}, g_{k}\right)_{L_{2}\left(\Gamma_{k}\right)}=0$. Therefore, we conclude

$$
\begin{aligned}
\left(\varepsilon V_{\beta}+I\right)_{\mid \Gamma_{k}} \sigma_{2}^{k}=g_{k}\left(g_{k}, g_{k}\right)_{L_{2}\left(\Gamma_{k}\right)}^{-1} & \\
& \cdot\left\{\varepsilon\left(V_{\beta} \sigma_{2}^{k}, g_{k}\right)_{L_{2}\left(\Gamma_{k}\right)}+\left(V_{\beta} \sigma_{1}^{k}, g_{k}\right)_{L_{2}\left(\Gamma_{k}\right)}\right\}-V_{\beta} \sigma_{1}^{k} \in H^{-\beta / 2}\left(\Gamma_{k}\right) .
\end{aligned}
$$

For every fixed $\varepsilon>0$ sufficiently small, the mapping $\varepsilon V_{\beta}+I: \mathcal{H}_{\varepsilon} \rightarrow \mathcal{H}_{\varepsilon}^{\prime}$ defines an isomorphism due to (3.5) and the Lax-Milgram lemma. Therefore, (6.10) amounts to the estimate

$$
\begin{aligned}
\left\|\boldsymbol{\sigma}_{2}\right\|_{\mathcal{H}_{\varepsilon}} & \leq c\left\|\left(g_{k}\left(g_{k}, g_{k}\right)_{L_{2}\left(\Gamma_{k}\right)}^{-1}\left\{\varepsilon\left(V_{\beta} \sigma_{2}^{k}, g_{k}\right)_{L_{2}\left(\Gamma_{k}\right)}+\left(V_{\beta} \sigma_{1}^{k}, g_{k}\right)_{L_{2}\left(\Gamma_{k}\right)}\right\}\right)_{k \in I}-V_{\beta} \boldsymbol{\sigma}_{1}\right\|_{\mathcal{H}_{\varepsilon}^{\prime}} \\
& \leq c^{\prime} \varepsilon\left(\sum_{k \in I}\left(V_{\beta} \sigma_{2}^{k}, g_{k}\right)_{L_{2}\left(\Gamma_{k}\right)}\right)+c^{\prime}\left\|V_{\beta} \boldsymbol{\sigma}_{1}\right\|_{H^{-\beta / 2}(\Gamma)}
\end{aligned}
$$


with a constant $c^{\prime}$ depending on $\Gamma$ and $\mathbf{g}$ due to (6.5) but not on $\varepsilon$. With $\mathbf{g} \in H^{\beta}(\Gamma)$ and $V_{\beta}$ being a pseudodifferential operator of order $\beta$ on $\Gamma$, we further have

$$
\left|\sum_{k \in I}\left(V_{\beta} \sigma_{2}^{k}, g_{k}\right)_{L_{2}\left(\Gamma_{k}\right)}\right| \leq c^{\prime \prime}\left\|\boldsymbol{\sigma}_{2}\right\|_{L_{2}(\Gamma)}\|\mathbf{g}\|_{H^{\beta}(\Gamma)},
$$

implying that

$$
\begin{aligned}
\left\|\boldsymbol{\sigma}_{2}\right\| \|_{\mathcal{H}_{\varepsilon}} & \leq c^{\prime \prime \prime} \varepsilon\left\|\boldsymbol{\sigma}_{2}\right\|_{L_{2}(\Gamma)}+c_{\mathbf{v}}^{\prime}\left\|\boldsymbol{\sigma}_{1}\right\|_{H^{\beta / 2}(\Gamma)} \\
& \leq c^{\prime \prime \prime} \varepsilon\left\|\boldsymbol{\sigma}_{2}\right\|_{\mathcal{H}_{\varepsilon}}+c_{\mathbf{v}}^{\prime}\left\|\boldsymbol{\sigma}_{1}\right\|_{H^{\beta / 2}(\Gamma)} .
\end{aligned}
$$

Consequently, since the constants do not depend on $\varepsilon$, there exists an $\varepsilon_{0}>0$ such that

$$
\left\|\boldsymbol{\sigma}_{2}\right\|_{\mathcal{H}_{\varepsilon}} \leq c_{\mathbf{V}}\left\|\boldsymbol{\sigma}_{1}\right\|_{H^{\beta / 2}(\Gamma)} \text { for all } 0<\varepsilon<\varepsilon_{0}
$$

and $c_{\mathbf{v}}$ independent of $\varepsilon$.

With $\mathbf{f} \in H^{\beta / 2}(\Gamma), \mathbf{g} \in H^{\frac{3}{2} \beta}(\Gamma)$, we find $\boldsymbol{\sigma}_{0} \in H^{\frac{3}{2} \beta}(\Gamma)$ and $\boldsymbol{\sigma}_{1} \in H^{\beta / 2}(\Gamma)$. Hence, (6.4) is justified which completes the proof of Theorem 6.1.

With the help of the previous theorem, we immediately find the following asymptotic behaviour of the minimizer $\boldsymbol{\sigma}_{\varepsilon}^{\star}$ if $\varepsilon$ tends to zero.

Corollary 6.2. Under the assumptions in Theorem 6.1, we find that

$$
\left\|\boldsymbol{\sigma}_{\varepsilon}^{\star}-\sigma_{0}\right\|_{L_{2}(\Gamma)} \leq c \varepsilon \stackrel{\varepsilon \rightarrow 0}{\longrightarrow} 0
$$

with some constant $c$, independent of $\boldsymbol{\sigma}_{0}$ and $\varepsilon>0$, where $\boldsymbol{\sigma}_{\varepsilon}^{\star}$ is the minimizer (6.4) of (6.1) for $\varepsilon>0$.

Proof. Since $\boldsymbol{\sigma}_{\varepsilon}^{\star}=\boldsymbol{\sigma}_{0}+\varepsilon \boldsymbol{\sigma}_{1}+\varepsilon^{2} \boldsymbol{\sigma}_{2}$, with (6.4) we find

$$
\left\|\boldsymbol{\sigma}_{\varepsilon}^{\star}-\sigma_{0}\right\|_{L_{2}(\Gamma)} \leq\left\|\boldsymbol{\sigma}_{\varepsilon}^{\star}-\sigma_{0}\right\|_{\mathcal{H}_{\varepsilon}} \leq c^{\prime}\left(\varepsilon+\varepsilon^{2}\right) \leq 2 c^{\prime} \varepsilon
$$

as proposed.

\section{Riesz Minimal ENERGY Without FINITE PART REDUCTION}

We consider next the punched hypersingular Riesz potential which is defined by integrating for a small $\delta>0$ only over $(\Gamma \times \Gamma) \backslash\{|\mathbf{x}-\mathbf{y}| \leq \delta\}$, i.e., by cutting out a set with $|\mathbf{x}-\mathbf{y}| \leq \delta$ near the singularity. Thus, the corresponding Riesz energy is defined as

$$
\stackrel{\circ}{\mathbf{J}}_{\delta}(\boldsymbol{\mu})=\iint_{\Gamma \times \Gamma \wedge 0<\delta \leq|\mathbf{x}-\mathbf{y}|}|\mathbf{x}-\mathbf{y}|^{\alpha-n} \mathrm{~d} \boldsymbol{\mu}(\mathbf{x}) \otimes \mathrm{d} \boldsymbol{\mu}(\mathbf{y})-2 \int_{\Gamma} \mathbf{f}(\mathbf{x}) \mathrm{d} \boldsymbol{\mu}(\mathbf{x}) .
$$

In view of Theorems 3.3, 3.5 and 4.1, the associated minimal Riesz energy problem is then equivalent to minimizing the punched functional

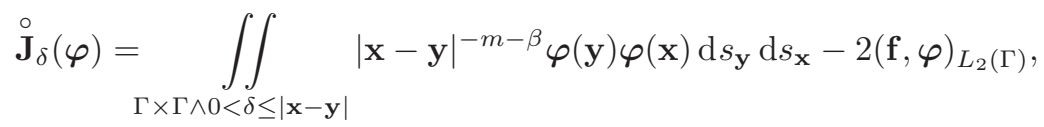

where $m=n-1$ and $\beta=1-\alpha \in(0,2)$, over the affine cone $\mathcal{K}(\Gamma, \mathbf{a}, \mathbf{g})$. Then, the measures satisfy $\mathrm{d} \boldsymbol{\mu}(\mathbf{x})=\boldsymbol{\varphi}(\mathbf{x}) \mathrm{d} s$ with $\mathrm{d} s$ being the surface measure on $\Gamma$.

For $\stackrel{\circ}{\mathbf{J}}_{\delta}(\varphi)$ one has the following monotonicity property.

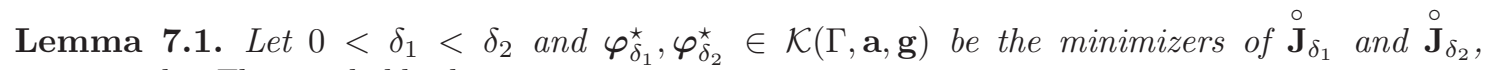
respectively. Then, it holds that

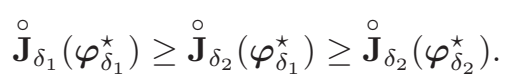


Proof. Since $\delta_{1}<\delta_{2}$, the minimizer $\varphi_{\delta_{1}}^{\star}=\sum_{j \in I} \alpha_{j} \varphi_{\delta_{1}}^{\star j}$ with $\varphi_{\delta_{1}}^{\star j} \geq 0$ is an admissible element for

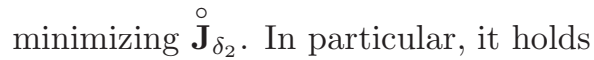

$$
\begin{aligned}
{\stackrel{\circ}{\mathbf{J}_{\delta_{1}}}}_{\left(\boldsymbol{\varphi}_{\delta_{1}}^{\star}\right)} & =\iint_{|\mathbf{x}-\mathbf{y}|>\delta_{1}}|\mathbf{x}-\mathbf{y}|^{-m-\beta} \boldsymbol{\varphi}_{\delta_{1}}^{\star}(\mathbf{x}) \boldsymbol{\varphi}_{\delta_{1}}^{\star}(\mathbf{y}) \mathrm{d} s_{\mathbf{y}} \mathrm{d} s_{\mathbf{x}}-2\left(\mathbf{f}, \boldsymbol{\varphi}_{\delta_{1}}^{\star}\right)_{L_{2}(\Gamma)} \\
& \geq \iint_{|\mathbf{x}-\mathbf{y}|>\delta_{2}}|\mathbf{x}-\mathbf{y}|^{-m-\beta} \boldsymbol{\varphi}_{\delta_{1}}^{\star}(\mathbf{x}) \boldsymbol{\varphi}_{\delta_{1}}^{\star}(\mathbf{y}) \mathrm{d} s_{\mathbf{y}} \mathrm{d} s_{\mathbf{x}}-2\left(\mathbf{f}, \boldsymbol{\varphi}_{\delta_{1}}^{\star}\right)_{L_{2}(\Gamma)}=\stackrel{\circ}{\mathbf{J}}_{\delta_{2}}\left(\boldsymbol{\varphi}_{\delta_{1}}^{\star}\right) .
\end{aligned}
$$

We further find

$$
\stackrel{\circ}{\mathbf{J}}_{\delta_{2}}\left(\boldsymbol{\varphi}_{\delta_{1}}^{\star}\right) \geq \inf \stackrel{\circ}{\mathbf{J}_{\delta_{2}}}(\boldsymbol{\varphi})=\stackrel{\circ}{\mathbf{J}}_{\delta_{2}}\left(\boldsymbol{\varphi}_{\delta_{2}}^{\star}\right)
$$

as proposed in (7.1).

In order to see the relation between $\stackrel{\circ}{\mathbf{J}}_{\delta}(\boldsymbol{\varphi})$ and $\mathbb{V}_{\mathbf{f}}(\boldsymbol{\varphi})$ in (4.2), let us introduce the compensating quadratic functional

$$
\mathbf{P}_{\delta}(\boldsymbol{\varphi}):=\int_{\Gamma}\left\{\text { p.f. } \int_{|\mathbf{x}-\mathbf{y}| \leq \delta}|\mathbf{x}-\mathbf{y}|^{-m-\beta} \boldsymbol{\varphi}(\mathbf{y}) \mathrm{d} s_{\mathbf{y}}\right\} \boldsymbol{\varphi}(\mathbf{x}) \mathrm{d} s_{\mathbf{x}} .
$$

Then, we obtain

$$
\begin{aligned}
\mathbf{P}_{\delta}(\boldsymbol{\varphi})+\stackrel{\circ}{\mathbf{J}}_{\delta}(\boldsymbol{\varphi}) & =\int_{\Gamma}\left\{\text { p.f. } \int_{\Gamma}|\mathbf{x}-\mathbf{y}|^{-m-\beta} \boldsymbol{\varphi}(\mathbf{y}) \mathrm{d} s_{\mathbf{y}}\right\} \boldsymbol{\varphi}(\mathbf{x}) \mathrm{d} s_{\mathbf{x}}-2(\mathbf{f}, \boldsymbol{\varphi})_{L_{2}(\Gamma)} \\
& =\left(V_{\beta} \boldsymbol{\varphi}, \boldsymbol{\varphi}\right)_{L_{2}(\Gamma)}-2(\mathbf{f}, \boldsymbol{\varphi})_{L_{2}(\Gamma)}=\mathbb{V}_{\mathbf{f}}(\boldsymbol{\varphi}),
\end{aligned}
$$

and thus

$$
\stackrel{\circ}{\mathbf{J}} \delta_{\delta}(\boldsymbol{\varphi})=\mathbb{V}_{\mathbf{f}}(\boldsymbol{\varphi})-\mathbf{P}_{\delta}(\boldsymbol{\varphi})=\left(V_{\beta} \boldsymbol{\varphi}, \boldsymbol{\varphi}\right)_{L_{2}(\Gamma)}-2(\mathbf{f}, \boldsymbol{\varphi})_{L_{2}(\Gamma)}-\mathbf{P}_{\delta}(\boldsymbol{\varphi})
$$

For the corresponding functional $\mathbf{P}_{\delta}$, there holds

Lemma 7.2. Let $\varphi \in \mathcal{K}(\Gamma, \mathbf{a}, \mathbf{g})$. Then

$$
\mathbf{P}_{\delta}(\boldsymbol{\varphi})=-\frac{1}{\beta} \frac{1}{c_{m}} \delta^{-\beta}\|\boldsymbol{\varphi}\|_{L_{2}(\Gamma)}^{2}+\mathbf{P}_{\delta}^{\prime}(\boldsymbol{\varphi}),
$$

where $\mathbf{P}_{\delta}^{\prime}(\boldsymbol{\varphi})$ satisfies

$$
\left|\mathbf{P}_{\delta}^{\prime}(\boldsymbol{\varphi})\right| \leq c\|\varphi\|_{H^{\beta / 2}(\Gamma)}^{2}
$$

with a constant $c$ independent of $\delta$. Moreover

$$
\lim _{\delta \rightarrow 0} \mathbf{P}_{\delta}^{\prime}(\boldsymbol{\varphi})=0 \text { for every } \boldsymbol{\varphi} \in H^{\beta / 2}(\Gamma)
$$


Proof. Using Martensen's coordinates of $\Gamma$ in the vicinity of $\mathbf{x} \in \Gamma$ (see Theorem B.2 in Appendix B), for every $\varphi \in C^{\infty}(\Gamma)$, we have

$$
\begin{aligned}
& \mathbf{P}_{\delta}(\boldsymbol{\varphi})=\int_{\Gamma}\left\{\text { p.f. } \int_{0<r \leq \delta \wedge|\boldsymbol{\Theta}|=1} \boldsymbol{\varphi}(\mathbf{x}+r \boldsymbol{\Theta}) r^{-\beta-1} \mathrm{~d} r \wedge \mathrm{d} \boldsymbol{\omega}\right. \\
& \left.+\int_{0<r \leq \delta \wedge|\Theta|=1}(\mathbf{x}+r \boldsymbol{\Theta}) a(\mathbf{x}, r) r^{-\beta+1} \mathrm{~d} r \wedge \mathrm{d} \boldsymbol{\omega}\right\} \boldsymbol{\varphi}(\mathbf{x}) \mathrm{d} s_{\mathbf{x}} \\
& =\left\{\text { p.f. } \int_{0<r \leq \delta \wedge|\Theta|=1} r^{-\beta-1} \mathrm{~d} r \wedge \mathrm{d} \boldsymbol{\omega}\right\} \int_{\Gamma}|\boldsymbol{\varphi}(\mathbf{x})|^{2} \mathrm{~d} s_{\mathbf{x}} \\
& +\int_{\Gamma}\left\{\text { p.f. } \int_{0<r \leq \delta \wedge|\Theta|=1}\{\boldsymbol{\varphi}(\mathbf{x}+r \boldsymbol{\Theta})-\boldsymbol{\varphi}(\mathbf{x})\} r^{-\beta-1} \mathrm{~d} r \wedge \mathrm{d} \boldsymbol{\omega}\right\} \boldsymbol{\varphi}(\mathbf{x}) \mathrm{d} s_{\mathbf{x}} \\
& +\int_{\Gamma}\left\{\text { p.f. } \int_{0<r \leq \delta \wedge|\boldsymbol{\Theta}|=1} \boldsymbol{\varphi}(\mathbf{x}+r \boldsymbol{\Theta}) a(\mathbf{x}, r) r^{-\beta+1} \mathrm{~d} r \wedge \mathrm{d} \boldsymbol{\omega}\right\} \boldsymbol{\varphi}(\mathbf{x}) \mathrm{d} s_{\mathbf{x}} .
\end{aligned}
$$

Since

$$
\text { p.f. } \int_{0<r \leq \delta \wedge|\Theta|=1} r^{-\beta-1} \mathrm{~d} r \wedge \mathrm{d} \boldsymbol{\omega}=-\frac{1}{\beta} \frac{1}{c_{m}} \delta^{-\beta}
$$

and $\mathbf{P}_{\delta}(\boldsymbol{\varphi})$ in (7.2) is symmetric, we find

$$
\mathbf{P}_{\delta}(\boldsymbol{\varphi})=-\left(\beta c_{m}\right)^{-1} \delta^{-\beta}\|\varphi\|_{L_{2}(\Gamma)}^{2}+\mathbf{P}_{\delta}^{\prime}(\boldsymbol{\varphi})
$$

Herein, $\mathbf{P}_{\delta}^{\prime}(\varphi)$ is given by

$$
\begin{aligned}
2 \mathbf{P}_{\delta}^{\prime}(\boldsymbol{\varphi})= & \iint_{|\mathbf{x}-\mathbf{y}| \leq \delta}\{\boldsymbol{\varphi}(\mathbf{x})(\boldsymbol{\varphi}(\mathbf{y})-\boldsymbol{\varphi}(\mathbf{x}))+\boldsymbol{\varphi}(\mathbf{y})(\boldsymbol{\varphi}(\mathbf{x})-\boldsymbol{\varphi}(\mathbf{y}))\}|\mathbf{x}-\mathbf{y}|^{-\beta-m} \mathrm{~d} s_{\mathbf{y}} \mathrm{d} s_{\mathbf{x}} \\
& -\iint_{|\mathbf{x}-\mathbf{y}| \leq \delta}(\boldsymbol{\varphi}(\mathbf{y})-\boldsymbol{\varphi}(\mathbf{x})) a(\mathbf{y}, r) r^{-\beta+1} \mathrm{~d} r \wedge \mathrm{d} \boldsymbol{\omega} \boldsymbol{\varphi}(\mathbf{x}) \mathrm{d} s_{\mathbf{x}} \\
& -\iint_{|\mathbf{x}-\mathbf{y}| \leq \delta}(\boldsymbol{\varphi}(\mathbf{x})-\boldsymbol{\varphi}(\mathbf{y})) a(\mathbf{x}, r) r^{-\beta+1} \mathrm{~d} r \wedge \mathrm{d} \boldsymbol{\omega} \boldsymbol{\varphi}(\mathbf{y}) \mathrm{d} s_{\mathbf{y}} \\
& +\iint_{|\mathbf{x}-\mathbf{y}| \leq \delta} \boldsymbol{\varphi}(\mathbf{y}) a(\mathbf{x}, r) r^{-\beta+1} \mathrm{~d} r \wedge \mathrm{d} \boldsymbol{\omega} \boldsymbol{\varphi}(\mathbf{x}) \mathrm{d} s_{\mathbf{x}} \\
& +\iint_{|\mathbf{x}-\mathbf{y}| \leq \delta} \boldsymbol{\varphi}(\mathbf{x}) a(\mathbf{y}, r) r^{-\beta+1} \mathrm{~d} r \wedge \mathrm{d} \boldsymbol{\omega} \varphi(\mathbf{y}) \mathrm{d} s_{\mathbf{y}}
\end{aligned}
$$

We rewrite $\mathbf{P}_{\delta}^{\prime}(\varphi)$ according to

$$
\begin{aligned}
\mathbf{P}_{\delta}^{\prime}(\boldsymbol{\varphi})= & \frac{1}{2} \iint_{|\mathbf{x}-\mathbf{y}| \leq \delta}|\boldsymbol{\varphi}(\mathbf{x})-\boldsymbol{\varphi}(\mathbf{y})|^{2}|\mathbf{x}-\mathbf{y}|^{-\beta-m} \mathrm{~d} s_{\mathbf{y}} \mathrm{d} s_{\mathbf{x}} \\
& +\iint_{|\mathbf{x}-\mathbf{y}| \leq \delta} b(\mathbf{x}, \mathbf{y}) \boldsymbol{\varphi}(\mathbf{x}) \boldsymbol{\varphi}(\mathbf{y}) \mathrm{d} s_{\mathbf{y}} \mathrm{d} s_{\mathbf{x}}+\iint_{|\mathbf{x}-\mathbf{y}| \leq \delta} c(\mathbf{x}, \mathbf{y}) \boldsymbol{\varphi}(\mathbf{x})^{2} \mathrm{~d} s_{\mathbf{y}} \mathrm{d} s_{\mathbf{x}}
\end{aligned}
$$


where $b(\mathbf{x}, \mathbf{y})$ and $c(\mathbf{x}, \mathbf{y})$ are kernels which posess pseudohomogeneous expansions of degree - $\beta-$ $m+1$. This means that

$$
\begin{aligned}
\mathbf{P}_{\delta}^{\prime}(\boldsymbol{\varphi})= & \frac{1}{2} \int_{|\mathbf{x}-\mathbf{y}| \leq \delta}|\boldsymbol{\varphi}(\mathbf{x})-\boldsymbol{\varphi}(\mathbf{y})|^{2}|\mathbf{x}-\mathbf{y}|^{-\beta-m} \mathrm{~d} s_{\mathbf{y}} \mathrm{d} s_{\mathbf{x}} \\
& +\int_{\Gamma}\left(\mathbf{B}_{\beta-1} \boldsymbol{\varphi}\right)(\mathbf{x}) \boldsymbol{\varphi}(\mathbf{x}) \mathrm{d} s_{\mathbf{x}}+\int_{\Gamma}\left(\mathbf{C}_{\beta-1} 1\right) \boldsymbol{\varphi}(\mathbf{x})^{2} \mathrm{~d} s_{\mathbf{x}}
\end{aligned}
$$

with classical pseudodifferential operators $\mathbf{B}_{\beta-1}$ and $\mathbf{C}_{\beta-1}$ of degree $\beta-1$ on $\Gamma$. Since the constant charge 1 is a smooth function on $\Gamma$ and $\mathbf{B}_{\beta-1}: H^{\beta / 2}(\Gamma) \rightarrow H^{-\beta / 2+1}(\Gamma) \hookrightarrow H^{-\beta / 2}(\Gamma)$ for $\varphi \in$ $H^{\beta / 2}(\Gamma)$, we finally arrive at

$$
\left|\mathbf{P}_{\delta}^{\prime}(\boldsymbol{\varphi})\right| \leq c_{1}\|\boldsymbol{\varphi}\|_{H^{\beta / 2}(\Gamma)}^{2}+c_{2}\|\varphi\|_{L_{2}(\Gamma)}^{2} \leq c\|\varphi\|_{H^{\beta / 2}(\Gamma)}^{2},
$$

as proposed. ${ }^{1}$

In order to show (7.3), consider first $\varphi \in C^{\infty}(\Gamma)$ and use Taylor's expansion about $\mathrm{x} \in \Gamma$ in (7.4). Then, all the integrals on the right hand side are weakly singular tending to zero with $\delta \rightarrow 0$. For $\boldsymbol{\varphi} \in H^{\beta / 2}(\Gamma)$ approximate $\boldsymbol{\varphi}$ by $\boldsymbol{\varphi}_{\varepsilon} \in C^{\infty}(\Gamma)$, satisfying $\left\|\varphi-\varphi_{\varepsilon}\right\|_{H^{\beta / 2}(\Gamma)}<\varepsilon$. Then

$$
\begin{aligned}
& \left|\mathbf{P}_{\delta}^{\prime}(\boldsymbol{\varphi})-\mathbf{P}_{\delta}^{\prime}\left(\boldsymbol{\varphi}_{\varepsilon}\right)\right| \\
& \leq \frac{1}{2} \iint_{|\mathbf{x}-\mathbf{y}| \leq \delta}\left\{|\boldsymbol{\varphi}(\mathbf{x})-\boldsymbol{\varphi}(\mathbf{y})|^{2}-\left|\boldsymbol{\varphi}_{\varepsilon}(\mathbf{x})-\boldsymbol{\varphi}_{\varepsilon}(\mathbf{y})\right|^{2}\right\}|\mathbf{x}-\mathbf{y}|^{-\beta-m} \mathrm{~d} s_{\mathbf{y}} \mathrm{d} s_{\mathbf{x}} \\
& \quad+\left|\left(\mathbf{B}_{\beta-1} \boldsymbol{\varphi}, \boldsymbol{\varphi}\right)_{L_{2}(\Gamma)}-\left(\mathbf{B}_{\beta-1} \boldsymbol{\varphi}_{\varepsilon}, \boldsymbol{\varphi}_{\varepsilon}\right)_{L_{2}(\Gamma)}\right| \\
& \quad+\left\|\mathbf{C}_{\beta-1} 1\right\|_{L_{2}(\Gamma)}\left|\|\boldsymbol{\varphi}\|_{L_{2}(\Gamma)}^{2}-\left\|\boldsymbol{\varphi}_{\varepsilon}\right\|_{L_{2}(\Gamma)}^{2}\right| .
\end{aligned}
$$

With

$$
\|\varphi\|_{H^{\beta / 2}(\Gamma)}^{2}-\left\|\boldsymbol{\varphi}_{\varepsilon}\right\|_{H^{\beta / 2}(\Gamma)}^{2} \leq 3\|\varphi\|_{H^{\beta / 2}(\Gamma)}\left\|\boldsymbol{\varphi}-\boldsymbol{\varphi}_{\varepsilon}\right\|_{H^{\beta / 2}(\Gamma)}
$$

for $\left\|\boldsymbol{\varphi}_{\varepsilon}\right\|_{H^{\beta / 2}(\Gamma)} \leq 2\|\boldsymbol{\varphi}\|_{H^{\beta / 2}(\Gamma)}^{2}$, one has

$$
\begin{aligned}
& \left|\mathbf{P}_{\delta}^{\prime}(\boldsymbol{\varphi})-\mathbf{P}_{\delta}^{\prime}\left(\boldsymbol{\varphi}_{\varepsilon}\right)\right| \\
& \leq \frac{3}{2}\left\{\iint_{|\mathbf{x}-\mathbf{y}| \leq \delta}\left|\boldsymbol{\varphi}(\mathbf{x})-\boldsymbol{\varphi}_{\varepsilon}(\mathbf{x})\right|\left|\boldsymbol{\varphi}(\mathbf{y})-\boldsymbol{\varphi}_{\varepsilon}(\mathbf{y})\right||\mathbf{x}-\mathbf{y}|^{-\beta-m} \mathrm{~d} s_{\mathbf{y}} \mathrm{d} s_{\mathbf{x}}\right\}^{\frac{1}{2}}\|\boldsymbol{\varphi}\|_{H^{\beta / 2}(\Gamma)} \\
& \quad+\left|\left(\mathbf{B}_{\beta-1}\left(\boldsymbol{\varphi}-\boldsymbol{\varphi}_{\varepsilon}\right), \boldsymbol{\varphi}\right)_{L_{2}(\Gamma)}\right|+\left|\left(\mathbf{B}_{\beta-1} \boldsymbol{\varphi}_{\varepsilon},\left(\boldsymbol{\varphi}-\boldsymbol{\varphi}_{\varepsilon}\right)\right)_{L_{2}(\Gamma)}\right| \\
& \quad+3\left\|\mathbf{C}_{\beta-1} 1\right\|_{L_{2}(\Gamma)}\|\boldsymbol{\varphi}\|_{H^{\beta / 2}(\Gamma)}\left\|\boldsymbol{\varphi}-\boldsymbol{\varphi}_{\varepsilon}\right\|_{H^{\beta / 2}(\Gamma)} \\
& \leq c\|\boldsymbol{\varphi}\|_{H^{\beta / 2}(\Gamma)}\left\|\boldsymbol{\varphi}-\boldsymbol{\varphi}_{\varepsilon}\right\|_{H^{\beta / 2}(\Gamma)} \leq c \varepsilon\|\boldsymbol{\varphi}\|_{H^{\beta / 2}(\Gamma)} .
\end{aligned}
$$

Then

$$
\varlimsup_{\delta \rightarrow 0}\left|\mathbf{P}_{\delta}^{\prime}(\boldsymbol{\varphi})\right| \leq c \varepsilon\|\boldsymbol{\varphi}\|_{H^{\beta / 2}(\Gamma)}
$$

for any $\varepsilon>0$ which implies (7.3).

Since

$$
\begin{aligned}
{\stackrel{\circ}{\mathbf{J}_{\delta}}(\boldsymbol{\varphi})} & =\iint_{0<\delta \leq|\mathbf{x}-\mathbf{y}|}|\mathbf{x}-\mathbf{y}|^{-\beta-m} \boldsymbol{\varphi}(\mathbf{y}) \boldsymbol{\varphi}(\mathbf{x}) \mathrm{d} s_{\mathbf{y}} \mathrm{d} s_{\mathbf{x}}-2(\mathbf{f}, \boldsymbol{\varphi})_{L^{2}(\Gamma)} \\
& =\frac{1}{\varepsilon}\|\boldsymbol{\varphi}\|_{L_{2}(\Gamma)}^{2}+\left(V_{\beta} \boldsymbol{\varphi}, \boldsymbol{\varphi}\right)_{L_{2}(\Gamma)}-2(\mathbf{f}, \boldsymbol{\varphi})_{L_{2}(\Gamma)}-\mathbf{P}_{\delta}^{\prime}(\boldsymbol{\varphi}),
\end{aligned}
$$

where $\varepsilon=\beta c_{m} \delta^{\beta} \rightarrow 0$ for $\delta \rightarrow 0$, Lemma 7.1 implies

\footnotetext{
${ }^{1}$ For $\beta=1, \mathbf{B}_{0}$ is a singular Mikhlin-Calderon integral operator with principal part $b(\mathbf{x}, \mathbf{x}) \Theta(\omega)$ which satisfies the Mikhlin condition $b(\mathbf{x}, \mathbf{x}) \int_{|\Theta|=1} \Theta(\omega) \mathrm{d} \boldsymbol{\omega}=0$.
} 
Corollary 7.3. Let $\beta_{0}<2$ and $\delta_{0}>0$ be given. Then, there exist positive constants $c, c^{\prime}>0$ such that the estimate

$$
\stackrel{\circ}{\mathbf{J}}_{\delta}(\boldsymbol{\varphi})-\frac{1}{\beta} \frac{1}{c_{m}} \delta^{-\beta}\|\boldsymbol{\varphi}\|_{L_{2}(\Gamma)}^{2} \leq c\|\boldsymbol{\varphi}\|_{H^{\beta / 2}(\Gamma)}^{2}+2\|\mathbf{f}\|_{L_{2}(\Gamma)}\|\boldsymbol{\varphi}\|_{L_{2}(\Gamma)} \leq c^{\prime}
$$

holds uniformly for $\varphi \in \mathcal{K}^{\beta / 2}(\Gamma), 0<\delta \leq \delta_{0}$ and $0 \leq \beta \leq \beta_{0}$.

The functional (7.5) coincides with the functional from (6.1) except for the perturbation term $\mathbf{P}_{\delta}^{\prime}(\varphi)$. Hence, we can proceed in complete analogy to the proof of Theorem 6.1 .

The Lagrangian to the punched energy functional reads as

$$
\begin{aligned}
\stackrel{\circ}{\mathbf{L}}_{\lambda}(\boldsymbol{\sigma}):=\frac{1}{2}\left(\left(\varepsilon V_{\beta} \boldsymbol{\sigma}+\boldsymbol{\sigma}\right), \boldsymbol{\sigma}\right)_{L_{2}(\Gamma)}-\varepsilon \mathbf{P}_{\delta}^{\prime}(\boldsymbol{\sigma})-\varepsilon(\mathbf{f}, \boldsymbol{\sigma})_{L_{2}(\Gamma)} \\
+\sum_{j \in I} \alpha_{j} \lambda_{j}\left(a^{j}-\left(g_{j}, \sigma^{j}\right)_{L_{2}\left(\Gamma_{j}\right)}\right),
\end{aligned}
$$

where the first order necessary optimality condition is given by

$$
\begin{gathered}
\partial_{\sigma^{k}} \stackrel{\circ}{\mathbf{L}}_{\lambda}(\boldsymbol{\sigma})=\varepsilon \alpha_{k} V_{\beta} \sigma_{\varepsilon}^{k}+\alpha_{k} \sigma_{\varepsilon}^{k}-\varepsilon \alpha_{k} \mathbf{P}_{\delta}^{\prime \prime} \sigma_{\varepsilon}^{k}-\varepsilon \alpha_{k} f_{k}-\alpha_{k} \lambda_{k} g_{k}=0, \\
\left(g_{k}, \sigma_{\varepsilon}^{k}\right)_{L_{2}\left(\Gamma_{k}\right)}=a^{k}, k \in I .
\end{gathered}
$$

Again, $\boldsymbol{\lambda} \neq \mathbf{0}$ since the constraints (6.3) are active. Here,

$$
\begin{array}{r}
\mathbf{P}_{\delta}^{\prime \prime} \sigma_{\varepsilon}^{k}=\partial_{\sigma_{\varepsilon}^{k}} \mathbf{P}_{\delta}^{\prime}\left(\boldsymbol{\sigma}_{\varepsilon}\right)=\int_{|\mathbf{x}-\mathbf{y}| \leq \delta}\left(\sigma_{\varepsilon}^{k}(\mathbf{y})-\sigma_{\varepsilon}^{k}(\mathbf{x})\right)|\mathbf{x}-\mathbf{y}|^{-m-\beta} \mathrm{d} s_{\mathbf{y}} \\
+\mathbf{B}_{\beta-1} \sigma_{\varepsilon}^{k}+\mathbf{B}_{\beta-1}^{\star} \sigma_{\varepsilon}^{k}+2 c_{\beta-1}(\mathbf{x}) \sigma_{\varepsilon}^{k}(\mathbf{x})
\end{array}
$$

with

$$
c_{\beta-1}(\mathbf{x})=\int_{\mathbf{y} \in \Gamma \wedge|\mathbf{x}-\mathbf{y}| \leq \delta} c(\mathbf{x}, \mathbf{y}) \mathrm{d} s_{\mathbf{y}}
$$

and $\mathbf{B}_{\beta-1}$ and $\mathbf{B}_{\beta-1}^{\star}$ being bounded linear operators:

$$
\begin{aligned}
\mathbf{B}_{\beta-1} \sigma_{\varepsilon}^{k}= & \int_{\mathbf{y} \in \Gamma \wedge|\cdot-\mathbf{y}| \leq \delta} b(\cdot, \mathbf{y}) \sigma_{\varepsilon}^{k}(\mathbf{y}) \mathrm{d} s_{\mathbf{y}}: H^{\frac{3}{2} \beta}(\Gamma) \rightarrow H^{\frac{1}{2} \beta}(\Gamma), \\
\mathbf{B}_{\beta-1}^{\star} \sigma_{\varepsilon}^{k}= & \int_{\mathbf{y} \in \Gamma \wedge|\cdot-\mathbf{y}| \leq \delta} b(\mathbf{y}, \cdot) \sigma_{\varepsilon}^{k}(\mathbf{y}) \mathrm{d} s_{\mathbf{y}}: H^{\frac{1}{2} \beta}(\Gamma) \rightarrow H^{-\frac{1}{2} \beta}(\Gamma) .
\end{aligned}
$$

Under the assumptions of Theorem 6.1 and as in the proof of Theorem 6.1 , we finally obtain the asymptotic expansion of the minimizer $\boldsymbol{\sigma}_{\varepsilon}^{\star}$ as well as of the Lagrangian multipliers:

$$
\boldsymbol{\sigma}_{\varepsilon}^{\star}=\boldsymbol{\sigma}_{0}+\varepsilon \boldsymbol{\sigma}_{1}+\varepsilon^{2} \boldsymbol{\sigma}_{2} \text { satisfying }\left\|\boldsymbol{\sigma}_{j}\right\|_{\mathcal{H}_{\varepsilon}} \leq c, j=0,1,2,
$$

and $\boldsymbol{\lambda}=\boldsymbol{\lambda}_{0}+\varepsilon \boldsymbol{\lambda}_{1}+\varepsilon^{2} \boldsymbol{\lambda}_{2}$ where

$$
\left(g_{k}, \sigma_{\varepsilon}^{k}\right)_{L_{2}\left(\Gamma_{k}\right)}=a^{k}, k \in I
$$

It turns out that $\sigma_{0}^{k}$ and $\lambda_{0}^{k}$ are exactly the same as in (6.7). Moreover, we have to replace $V_{\beta}$ by $\left(V_{\beta}-\mathbf{P}_{\delta}^{\prime \prime}\right)$ in the equations (6.8) and (6.9), (6.10). Note that $\boldsymbol{\sigma}_{0} \in H^{\frac{3}{2} \beta}(\Gamma) \hookrightarrow H^{\frac{1}{2} \beta}(\Gamma)$ and, hence, $\mathbf{P}_{\delta}^{\prime \prime} \sigma_{0}^{k}$ for $\delta \rightarrow 0$ tends to zero in $H^{\beta / 2}(\Gamma)$ due to $(7.3)$. (If $\boldsymbol{\sigma}_{0}, \boldsymbol{\sigma}_{1} \in C^{1}(\Gamma)$ for $0<\beta<1$ or $\boldsymbol{\sigma}_{0}, \boldsymbol{\sigma}_{1} \in C^{2}(\Gamma)$ for $1 \leq \beta \leq 2$, then $\mathbf{P}_{\delta}^{\prime \prime} \sigma_{0}^{k}$ and $\mathbf{P}_{\delta}^{\prime \prime} \sigma_{1}^{k}=\mathcal{O}\left(\delta^{1-\beta}\right)$, respectively $\mathcal{O}\left(\delta^{2-\beta}\right)$.)

Collecting these results, we have for the punched energy Riesz minimum problem the following result:

Theorem 7.4. Under the same assumptions as for Theorem 6.1, the minimization problem

$$
\frac{1}{2} \underset{\substack{0<\delta \leq|\mathbf{x}-\mathbf{y}| \\ \mathbf{x}, \mathbf{y} \in \Gamma}}{ }|\mathbf{x}-\mathbf{y}|^{\alpha-n} \boldsymbol{\sigma}(\mathbf{y}) \boldsymbol{\sigma}(\mathbf{x}) \mathrm{d} s_{\mathbf{y}} \mathrm{d} s_{\mathbf{x}}-\int_{\Gamma} \mathbf{f}(\mathbf{x}) \boldsymbol{\sigma}(\mathbf{x}) \mathrm{d} s_{\mathbf{x}} \rightarrow \min ,
$$


subject to (6.2), has for every $\delta>0$ a unique solution $\boldsymbol{\sigma}_{\varepsilon}^{\star}$. It admits for $\varepsilon=\beta c_{m} \delta^{\beta}>0$ the asymptotic expansion

$$
\boldsymbol{\sigma}_{\varepsilon}^{\star}=\boldsymbol{\sigma}_{0}+\varepsilon \boldsymbol{\sigma}_{1}+\varepsilon^{2} \boldsymbol{\sigma}_{2} \text { satisfying }\left\|\boldsymbol{\sigma}_{j}\right\|_{\mathcal{H}_{\varepsilon}} \leq c, j=0,1,2
$$

with a constant $c$ independent of $\varepsilon$. In particular, with $\boldsymbol{\sigma}_{\varepsilon}^{\star}=\left(\alpha_{k} \sigma_{0}^{k}+\alpha_{k} \sigma_{1}^{k}+\alpha_{k} \sigma_{2}^{k}\right)_{k \in I}$ there holds

$$
\begin{aligned}
\sigma_{0}^{k} & =g_{k} a^{k}\left(g_{k}, g_{k}\right)_{L_{2}\left(\Gamma_{k}\right)}^{-1}, \\
\sigma_{1}^{k} & =\lambda_{1}^{k} g_{k}-\left(V_{\beta}-\mathbf{P}_{\delta}^{\prime \prime}\right) \sigma_{0}^{k}+f_{k},
\end{aligned}
$$

where

$$
\lambda_{1}^{k}=\left(g_{k}, g_{k}\right)^{-1}\left(\left(V_{\beta}-\mathbf{P}_{\delta}^{\prime \prime}\right) \sigma_{0}^{k}-f_{k}, g_{k}\right)_{L_{2}\left(\Gamma_{k}\right)}
$$

and

$$
\sigma_{2}^{k}=\left(I+\varepsilon\left(V_{\beta}-P_{j}^{\prime \prime}\right)\right)^{-1}\left\{\lambda_{2}^{k} g_{k}-\left(V_{\beta}-P_{j}^{\prime \prime}\right) \sigma_{1}^{k}\right\}
$$

with

$$
\lambda_{2}^{k}=\left(g_{k}, g_{k}\right)_{L_{2}\left(\Gamma_{k}\right)}^{-1}\left\{\left(V_{\beta}-P_{j}^{\prime \prime}\right) \sigma_{1}^{k}+\varepsilon\left(V_{\beta}-P_{j}^{\prime \prime}\right) \sigma_{2}^{k}+\sigma_{2}^{k}\right\} .
$$

Corollary 7.5. For $\delta \rightarrow 0$ and $\varepsilon=\beta c_{m} \delta^{\beta}$ one finds

$$
\left\|\boldsymbol{\sigma}_{\varepsilon}^{\star}-\boldsymbol{\sigma}_{0}\right\|_{L_{2}(\Gamma)} \leq c \varepsilon \stackrel{\delta \rightarrow 0}{\longrightarrow} 0
$$

with some constant $c$, independent of $\boldsymbol{\sigma}_{0}$ and $\varepsilon>0$, where $\boldsymbol{\sigma}_{\varepsilon}^{\star}$ is the minimizer (7.6). Moreover, it holds

$$
\stackrel{\circ}{\mathbf{J}}_{\delta}\left(\boldsymbol{\sigma}_{\varepsilon}^{\star}\right)=\left(\mathbb{V}_{\beta}\left(\boldsymbol{\sigma}_{\varepsilon}^{\star}\right), \boldsymbol{\sigma}_{\varepsilon}^{\star}\right)_{L_{2}(\Gamma)}-P_{\delta}^{\prime}\left(\boldsymbol{\sigma}_{\varepsilon}^{\star}\right)+\frac{1}{\beta} \frac{1}{c_{m}} \delta^{-\beta}\left\|\boldsymbol{\sigma}_{\varepsilon}^{\star}\right\|_{L_{2}(\Gamma)}^{2} \stackrel{\delta \rightarrow 0}{\longrightarrow} \infty .
$$

Proof. Due to (7.7), we obtain

$$
\left\|\boldsymbol{\sigma}_{\varepsilon}^{\star}-\boldsymbol{\sigma}_{0}\right\|_{L_{2}(\Gamma)} \leq\left\|\boldsymbol{\sigma}_{\varepsilon}^{\star}-\boldsymbol{\sigma}_{0}^{\star}\right\|_{\mathcal{H}_{\varepsilon}} \leq \varepsilon\left\|\boldsymbol{\sigma}_{1}\right\|_{\mathcal{H}_{\varepsilon}}+\varepsilon^{2}\left\|\boldsymbol{\sigma}_{2}\right\|_{\mathcal{H}_{\varepsilon}} \leq c\left(\varepsilon+\varepsilon^{2}\right) .
$$

The conditions (6.3) imply that $\sigma_{0}^{k}>0$ and, hence, $\left\|\boldsymbol{\sigma}_{\varepsilon}^{\star}\right\|_{L_{2}(\Gamma)} \geq \frac{1}{2}\left\|\boldsymbol{\sigma}_{0}\right\|_{L_{2}(\Gamma)}>0$ for all $\varepsilon$ with $0<\varepsilon \leq \varepsilon_{1}$ with some $\varepsilon_{1}>0$. Thus, there holds

$$
\begin{aligned}
\stackrel{\circ}{\mathbf{J}}_{\delta}\left(\boldsymbol{\sigma}_{\varepsilon}^{\star}\right) & =\left(\mathbb{V}_{\beta}\left(\boldsymbol{\sigma}_{\varepsilon}^{\star}\right), \boldsymbol{\sigma}_{\varepsilon}^{\star}\right)_{L_{2}(\Gamma)}-P_{\delta}^{\prime}\left(\boldsymbol{\sigma}_{\varepsilon}^{\star}\right)+\frac{1}{\beta} \frac{1}{c_{m}} \delta^{-\beta}\left\|\boldsymbol{\sigma}_{\varepsilon}^{\star}\right\|_{L_{2}(\Gamma)}^{2} \\
& \geq \frac{1}{2 \beta} \frac{1}{c_{m}} \delta^{-\beta}\left\|\boldsymbol{\sigma}_{0}\right\|_{L_{2}(\Gamma)}^{2}-c\left\|\boldsymbol{\sigma}_{\varepsilon}^{\star}\right\|_{\mathcal{H}_{\varepsilon}}^{2}
\end{aligned}
$$

with uniformly bounded $\left\|\boldsymbol{\sigma}_{\varepsilon}^{\star}\right\|_{\mathcal{H}_{\varepsilon}}^{2}$. Hence, $\delta \rightarrow 0$ implies (7.8).

Remark 7.6. For the torus $\Gamma_{1}$ in $\mathbb{R}^{3}$, considered in [6] and [10], where $\mathbf{f} \in H^{\beta / 2}(\Gamma), a^{1}>0$, $g_{1}=1$, the minimizers $\boldsymbol{\sigma}_{\varepsilon}^{\star}$ of the punched minimization problem tend to the constant charge $\boldsymbol{\sigma}_{\varepsilon}^{\star} \rightarrow \boldsymbol{\sigma}_{0}:=\frac{1}{|\Gamma|} a^{1}$.

\section{Appendix A. Explicit CALCULATION OF PARTICULAR PARTIE FINIE INTEGRALS}

In this appendix, we shall compute the partie finie integrals which define the functions $h(\mathbf{x})$ and $\mathbf{h}(\mathbf{x})$ from (3.2) and (3.3), respectively.

Lemma A.1. (i) Let $-1<\alpha<1, \Gamma \in C^{\infty}$ and $\varphi \in C^{\infty}(\Gamma)$. Then, one has

$$
\begin{aligned}
& \lim _{\delta \rightarrow 0} \text { p.f. } \int_{|\mathbf{x}-\mathbf{y}|>\delta>0}|\mathbf{x}-\mathbf{y}|^{-\beta-(n-1)}\{\boldsymbol{\varphi}(\mathbf{y})-\boldsymbol{\varphi}(\mathbf{x})\} \mathrm{d} s_{\mathbf{y}} \\
& =\text { p.v. } \int_{\Gamma \backslash\{\mathbf{x}\}}|\mathbf{x}-\mathbf{y}|^{-\beta-(n-1)}\{\boldsymbol{\varphi}(\mathbf{y})-\boldsymbol{\varphi}(\mathbf{x})\} \mathrm{d} s_{\mathbf{y}},
\end{aligned}
$$

where p.v. denotes the Mikhlin-Calderon principal value integral. 
(ii) The function $h(\mathbf{x})$ from (3.2) is given by

$$
\begin{array}{r}
h(\mathbf{x})=\int_{\mathbf{y} \in \Gamma \wedge|\mathbf{x}-\mathbf{y}| \leq c}\left\{|\mathbf{x}-\mathbf{y}|^{-\beta-(n-1)}-r^{-\beta-(n-1)}\right\} \mathrm{d} s_{\mathbf{y}}-\left(\beta c_{n}\right)^{-1} c^{-\beta} \\
- \text { p.v. } \int_{\mathbf{y} \in \Gamma \wedge|\mathbf{x}-\mathbf{y}| \leq c} r^{-\beta-(n-1)}\left\{r^{n-2} \mathrm{~d} r \mathrm{~d} \boldsymbol{\omega}-\mathrm{d} s_{\mathbf{y}}\right\} \\
+\int_{\mathbf{y} \in \Gamma \wedge|\mathbf{x}-\mathbf{y}| \geq c}|\mathbf{x}-\mathbf{y}|^{-\beta-(n-1)} \mathrm{d} s_{\mathbf{y}}
\end{array}
$$

with any $c>0$ sufficiently small.

(iii) For the function $\mathbf{h}(\mathbf{x})$ from (3.3), one obtains

$$
\begin{array}{r}
\mathbf{h}(\mathbf{x})=\text { p.v. } \quad \int_{\mathbf{y} \in \Gamma \wedge 0<|\mathbf{x}-\mathbf{y}| \leq c}\left\{|\mathbf{x}-\mathbf{y}|^{-\beta-(n-1)}(\mathbf{y}-\mathbf{x})-r^{-\beta-(n-1)}(\mathbf{y}-\mathbf{x})\right\} \mathrm{d} s_{\mathbf{y}} \\
- \text { p.v. } \quad \int_{\mathbf{y} \in \Gamma \wedge|\mathbf{x}-\mathbf{y}| \leq c} r^{-\beta-(n-1)}\left\{(\mathbf{y}-\mathbf{x}) r^{n-2} \mathrm{~d} r \mathrm{~d} \boldsymbol{\omega}-(\mathbf{y}-\mathbf{x}) \mathrm{d} s_{\mathbf{y}}\right\} \\
+\int_{\mathbf{y} \in \Gamma \wedge 0<|\mathbf{x}-\mathbf{y}| \geq c}|\mathbf{x}-\mathbf{y}|^{-\beta-(n-1)}(\mathbf{y}-\mathbf{x}) \mathrm{d} s_{\mathbf{y}}
\end{array}
$$

with any $c>0$ sufficiently small.

Proof. (i) Locally on $\Gamma$ one has near $\mathbf{x} \in \Gamma$ :

$$
\begin{aligned}
\boldsymbol{\varphi}(\mathbf{y}) & =\boldsymbol{\varphi}(\mathbf{x})+(\mathbf{y}-\mathbf{x}) \cdot \nabla \boldsymbol{\varphi}(\mathbf{x})+\mathcal{O}\left(r^{2}\right), r=|\mathbf{x}-\mathbf{y}| \\
\boldsymbol{\Theta}(r, \boldsymbol{\omega}) & :=\frac{1}{r}(\mathbf{y}-\mathbf{x}) \text { for } \mathbf{x}, \mathbf{y} \in \Gamma \\
\boldsymbol{\Theta}(r, \boldsymbol{\omega}) & =\boldsymbol{\Theta}(0, \boldsymbol{\omega})+\mathcal{O}(r), \int_{|\Theta|=1} \boldsymbol{\Theta}(0, \boldsymbol{\omega}) \mathrm{d} \boldsymbol{\omega}=0 \\
\mathrm{~d} s_{\mathbf{y}} & =r^{n-2}\left(1+\mathcal{O}\left(r^{2}\right)\right) \mathrm{d} r \mathrm{~d} \boldsymbol{\omega} .
\end{aligned}
$$

$|\boldsymbol{\Theta}(0, \boldsymbol{\omega})|=1$ describes the $(n-2)$-dimensional unit sphere $\mathbb{S}^{n-2}$ and $\boldsymbol{\omega}$ is its polar coordinate with $\mathrm{d} \boldsymbol{\omega}$ its $(n-2)$-dimensional surface measure. Consequently, with an appropriate constant $c>0$ 
depending on $\Gamma$, one has

$$
\begin{aligned}
& \lim _{\delta \rightarrow 0} \text { p.f. } \int_{\mathbf{y} \in \Gamma \wedge 0<\delta<|\mathbf{x}-\mathbf{y}|}|\mathbf{x}-\mathbf{y}|^{-\beta-(n-1)}\{\boldsymbol{\varphi}(\mathbf{y})-\boldsymbol{\varphi}(\mathbf{x})\} \mathrm{d} s_{\mathbf{y}} \\
& =\lim _{\delta \rightarrow 0+} \text { p.f. } \int_{\mathbf{y} \in \Gamma \wedge 0<\delta<|\mathbf{x}-\mathbf{y}| \leq c}|\mathbf{x}-\mathbf{y}|^{-\beta-(n-1)} \\
& \times\{(\mathbf{y}-\mathbf{x}) \cdot \nabla \varphi(\mathbf{x})+\{\varphi(\mathbf{y})-\varphi(\mathbf{x})-(\mathbf{y}-\mathbf{x}) \cdot \nabla \varphi(\mathbf{x})\}\} \mathrm{d} s_{\mathbf{y}} \\
& +\int_{\mathbf{y} \in \Gamma \wedge|\mathbf{x}-\mathbf{y}| \geq c}|\mathbf{x}-\mathbf{y}|^{-\beta-(n-1)}\{\boldsymbol{\varphi}(\mathbf{y})-\boldsymbol{\varphi}(\mathbf{x})\} \mathrm{d} s_{\mathbf{y}} \\
& =\lim _{\delta \rightarrow 0+} \text { p.f. } \int_{0<\delta \leq r \leq c} r^{-\beta} \mathrm{d} r \int_{|\boldsymbol{\Theta}|=1} \boldsymbol{\Theta} \mathrm{d} \boldsymbol{\omega}(\boldsymbol{\Theta}) \cdot \nabla \boldsymbol{\varphi}(\mathbf{x}) \\
& + \text { p.v. }\left\{\int_{0<|\mathbf{x}-\mathbf{y}| \leq c}\left\{|\mathbf{x}-\mathbf{y}|^{-\beta-(n-1)}(\mathbf{y}-\mathbf{x}) \mathrm{d} s_{\mathbf{y}}-r^{-\beta-1}(\mathbf{y}-\mathbf{x}) \mathrm{d} r \mathrm{~d} \boldsymbol{\omega}\right\}\right. \\
& \left.+\int_{0<|\mathbf{x}-\mathbf{y}| \leq c}|\mathbf{x}-\mathbf{y}|^{-\beta-(n-1)}\left\{\boldsymbol{\varphi}(\mathbf{y})-\boldsymbol{\varphi}(\mathbf{x})-(\mathbf{y}-\mathbf{x}) \nabla \boldsymbol{\varphi}(\mathbf{x}) \cdot \mathrm{d} s_{\mathbf{y}}\right\}\right\} \\
& +\int_{\mathbf{y} \in \Gamma \wedge|\mathbf{x}-\mathbf{y}| \geq c}|\mathbf{x}-\mathbf{y}|^{-\beta-(n-1)}\{\boldsymbol{\varphi}(\mathbf{y})-\boldsymbol{\varphi}(\mathbf{x})\} \mathrm{d} s_{\mathbf{y}} .
\end{aligned}
$$

The first integral on the right is zero because of $\int_{|\boldsymbol{\Theta}|=1} \boldsymbol{\Theta} \mathrm{d} \boldsymbol{\omega}=\mathbf{0}$. Whereas, the integrand of the remaining integral has at $\mathbf{y}=\mathbf{x}$ a weak singularity $|\mathbf{y}-\mathbf{x}|^{-\beta+1}$ with $-1<-\beta+1<1$ whose principal value integral exists. This follows by using e.g. Martensen's surface polar coordinates, cf. Theorem B.1 in Appendix B.

(ii) For the function

$$
h(\mathbf{x})=\lim _{\delta \rightarrow 0} \text { p.f. } \int_{0<\delta<|\mathbf{x}-\mathbf{y}| \wedge \mathbf{y} \in \Gamma}|\mathbf{x}-\mathbf{y}|^{-\beta-(n-1)} \mathrm{d} s_{\mathbf{y}},
$$

we find

$$
\begin{aligned}
h(\mathbf{x})= & \lim _{\delta \rightarrow 0} \text { p.f. } \int_{0<\delta<|\mathbf{x}-\mathbf{y}| \leq c}\left\{|\mathbf{x}-\mathbf{y}|^{-\beta-(n-1)}-r^{-\beta-(n-1)}\right\} \mathrm{d} s_{\mathbf{y}} \\
& +\lim _{\delta \rightarrow 0} \text { p.f. } \int_{0<\delta}^{c} r^{-\beta-(n-1)} r^{n-2} \mathrm{~d} r \int_{|\boldsymbol{\Theta}|=1} \mathrm{~d} \boldsymbol{\omega} \\
& -\lim _{\delta \rightarrow 0} \text { p.f. } \int_{0<\delta}^{c} \int_{|\boldsymbol{\Theta}|=1} r^{-\beta-(n-1)}\left\{r^{n-2} \mathrm{~d} r \mathrm{~d} \boldsymbol{\omega}-\mathrm{d} s_{\mathbf{y}}\right\} \\
& +\int_{c \leq|\mathbf{x}-\mathbf{y}| \wedge \mathbf{y} \in \Gamma}|\mathbf{x}-\mathbf{y}|^{-\beta-(n-1)} \mathrm{d} s_{\mathbf{y}} .
\end{aligned}
$$

If we choose Martensen's surface polar coordinates on $\Gamma$, then the integrand of the first integral on the right is identical zero due to $|\mathbf{x}-\mathbf{y}|=r$. For the second integral on the right, we have with 


$$
\begin{aligned}
& \int_{|\boldsymbol{\Theta}|=1} \mathrm{~d} \boldsymbol{\omega}=c_{n}^{-1} \text { that } \\
& \qquad \lim _{0<\delta \rightarrow 0} \text { p.f. } \int_{\delta}^{c} r^{\beta-1} \mathrm{~d} r \int_{|\boldsymbol{\Theta}|=1} \mathrm{~d} \boldsymbol{\omega}=\lim _{0<\delta \rightarrow 0} \text { p.f. } \frac{1}{c_{n}}\left\{\frac{\delta^{-\beta}}{\beta}-\frac{1}{\beta} c^{-\beta}\right\}=-\left(c_{n} \beta\right)^{-1} c^{-\beta} .
\end{aligned}
$$

For the third integral, Theorem B.2 in Appendix B implies

$$
r^{n-2} \mathrm{~d} r \mathrm{~d} \boldsymbol{\omega}-\mathrm{d} s_{\mathbf{y}}=\mathcal{O}\left(r^{n}\right) \mathrm{d} r \mathrm{~d} \boldsymbol{\omega} .
$$

Hence, the integrand is of order $\mathcal{O}\left(r^{-\beta+1}\right)$ and the integral exists as a principal value integral. Collecting these properties proves (A.1).

(iii) We proceed with respect to the vector-valued function

$$
\mathbf{h}(\mathbf{x})=\text { p.f. } \lim _{\delta \rightarrow 0} \int_{\mathbf{y} \in \Gamma \wedge 0<\delta<|\mathbf{x}-\mathbf{y}|}|\mathbf{x}-\mathbf{y}|^{-\beta-(n-1)}(\mathbf{y}-\mathbf{x}) \mathrm{d} s_{\mathbf{y}},
$$

in the same manner as for $h(\mathbf{x})$. Inserting $(\mathbf{y}-\mathbf{x})=r \boldsymbol{\Theta}$ for Martensen's surface polar coordinates, we have:

$$
\begin{aligned}
\mathbf{h}(\mathbf{x})= & \lim _{\delta \rightarrow 0} \text { p.f. } \int_{0<\delta<|\mathbf{x}-\mathbf{y}| \leq c}\left\{|\mathbf{x}-\mathbf{y}|^{-\beta-(n-1)}(\mathbf{y}-\mathbf{x})-r^{-\beta-(n-1)}(\mathbf{y}-\mathbf{x})\right\} \mathrm{d} s_{\mathbf{y}} \\
& +\lim _{\delta \rightarrow 0} \text { p.f. } \int_{0<\delta}^{c} r^{-\beta-(n-1)} r^{n-1} \mathrm{~d} r \int_{|\boldsymbol{\Theta}|=1} \boldsymbol{\Theta} \mathrm{d} \boldsymbol{\omega} \\
& -\lim _{\delta \rightarrow 0} \text { p.f. } \int_{\delta}^{c} \int_{|\boldsymbol{\Theta}|=1} r^{-\beta-n} \boldsymbol{\Theta}\left\{r^{n-2} \mathrm{~d} r \mathrm{~d} \boldsymbol{\omega}-\mathrm{d} s_{\mathbf{y}}\right\} \\
& +\int_{0 \leq|\mathbf{x}-\mathbf{y}| \wedge \mathbf{y} \in \Gamma}|\mathbf{x}-\mathbf{y}|^{-\beta-(n-1)}(\mathbf{y}-\mathbf{x}) \mathrm{d} s_{\mathbf{y}} .
\end{aligned}
$$

Here, the first integral on the right vanishes if Martensen's surface polar coordinates are used, and the second one vanishes because of $\int_{|\Theta|=1} \Theta \mathrm{d} \boldsymbol{\omega}=\mathbf{0}$. The third integral contains an integrand of order $\mathcal{O}\left(r^{\beta+2}\right)$. Hence, the integrand is bounded and the integral exists. Consequently, also (A.2) holds.

\section{Appendix B. Martensen's surface polar coordinates}

For $n=3$, surface polar coordinates have been introduced by Martensen in [16, Chapter 2.1]. A graphical illustration of these coordinates are found in Figure 2. We generalize this approach to arbitrary spatial dimension $n \geq 2$.

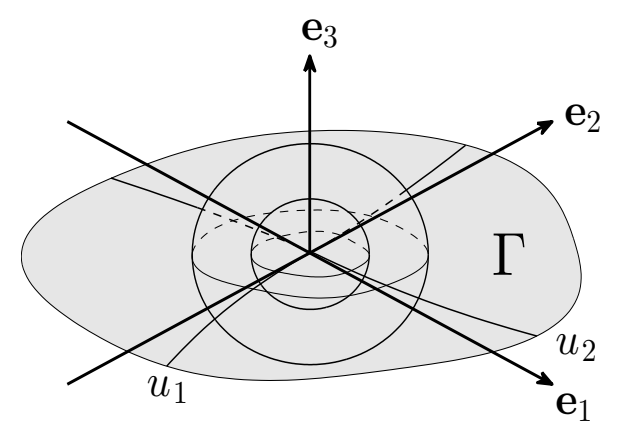

FigURE 2. Illustration of Martensen's surface polar coordinates. 
The given surface has locally the parametric representation $\Gamma: \mathbf{x}=\mathbf{x}(\mathbf{u}) \in \mathbb{R}^{n}, \mathbf{u}=$ $\left(u^{1}, \ldots, u^{n-1}\right) \in \mathbb{R}^{n-1}$. For $\mathbf{x}=\stackrel{\circ}{\mathbf{x}}=\mathbf{x}(\stackrel{\circ}{\mathbf{u}})$, define the family of $(n-2)$-dimensional manifolds (level sets) as $\left\{\mathbf{x} \in \mathcal{C}_{\varrho} \subset \Gamma\right.$ given by $\left.|\mathbf{x}-\stackrel{\circ}{\mathbf{x}}|=\varrho>0\right\}$, where $|\mathbf{x}-\stackrel{\circ}{\mathbf{x}}|$ denotes the Euclidian distance in $\mathbb{R}^{n}$ and $\varrho$ is the radial parameter. Let

then

$$
A(\mathbf{u}):=|\mathbf{x}(\mathbf{u})-\stackrel{\circ}{\mathbf{x}}|
$$

$$
A^{2}(\mathbf{u})=(\mathbf{x}(\mathbf{u})-\stackrel{\circ}{\mathbf{x}}) \cdot(\mathbf{x}(\mathbf{u})-\stackrel{\circ}{\mathbf{x}}),
$$

where $\cdot$ denotes the Euclidian scalar product in $\mathbb{R}^{n}$. In the local neighborhood of $\Gamma$, define

$$
\mathbf{x}(\mathbf{u})=\stackrel{\circ}{\mathbf{x}}+\mathbf{F}(\mathbf{u})+G(\mathbf{u}) \mathbf{n}(\mathbf{u})
$$

with $G(\mathbf{u})=(\mathbf{x}(\mathbf{u})-\stackrel{\circ}{\mathbf{x}}) \cdot \mathbf{n}(\mathbf{u})$, where $\mathbf{n}(\mathbf{u})$ denotes the (exterior) unit normal vector of $\Gamma$ at $\mathbf{x}=\mathbf{x}(\mathbf{u})$. Then, on $\Gamma$, the vector $\mathbf{F}(\mathbf{u})$ is tangential to $\Gamma$ at $\mathbf{x}(\mathbf{u})$,

$$
\mathbf{F} \cdot \mathbf{n}=0, \mathbf{F}=(\mathbf{x}(\mathbf{u})-\stackrel{\circ}{\mathbf{x}})-G(\mathbf{u}) \mathbf{n}(\mathbf{u}) \text { and } A^{2}=\mathbf{F} \cdot \mathbf{F}+G^{2} .
$$

For $\stackrel{\circ}{\mathbf{x}} \in \Gamma$ chosen, the surface polar coordinates then locally are given by the family of closed surfaces $\mathcal{C}_{\varrho}$, i.e., the level sets of the function $A(\mathbf{u})$, and curved radial rays on $\Gamma$ through $\stackrel{\circ}{\mathrm{x}}$ which are perpendicular to $\mathcal{C}_{\varrho}$ for constant $\varrho$. Let us denote such a radial ray curve by $\mathbf{c}_{\Theta} \in \Gamma$, given by $\mathbf{u}=\mathbf{u}(s), 0 \leq s$, where the parametric representation $\mathbf{x}(\mathbf{u}(s))$ at $s=0$ starts in $\stackrel{\circ}{\mathbf{x}}=\mathbf{x}(\stackrel{\circ}{\mathbf{u}})$ in the direction of the unit vector $\mathbf{e}(\Theta)=\mathbf{x}_{\mid i}(\stackrel{\circ}{\mathbf{u}}) \Theta^{i}, \mathbf{x}_{\mid i}=\frac{\partial \mathbf{x}}{\partial u^{i}}, i=1, \ldots, n-1 ; g_{j k}=\mathbf{x}_{\mid j} \cdot \mathbf{x}_{\mid k}$, $g_{j k} \Theta^{j} \Theta^{k}=1$. Hence, for the curve $\mathbf{c}_{\Theta}: \mathbf{x}=\mathbf{x}(\mathbf{u}(s))$, we require that

$$
\mathbf{x}_{\mid j}(\stackrel{\circ}{\mathbf{u}}) \frac{\mathrm{d} u^{j}}{\mathrm{~d} s}(\stackrel{\circ}{\mathbf{u}})=\mathbf{e}(\boldsymbol{\Theta}) .
$$

(Note that for $n=3$ one usually uses $\Theta_{1}=\cos \zeta$ and $\Theta_{2}=\sin \zeta$.)

Without restriction of generality, we assume in what follows that at $\stackrel{\circ}{\mathbf{x}}$ we have $g_{j k}(\stackrel{\circ}{\mathbf{u}})=\delta_{j k}$ (the Kronecker tensor). Since the radial ray curves $\mathbf{c}_{\Theta} \in \Gamma$ are perpendicular to the level sets, which implicitly are given by $A=\varrho=$ const, they satisfy the ordinary differential equations for fixed $\Theta$ :

$$
\frac{\partial \mathbf{c}_{\Theta}}{\partial s}=\left.\frac{\mathrm{d} \mathbf{x}}{\mathrm{d} s}\right|_{\mathbf{c}_{\Theta}}=\mathbf{x}_{\mid j} \frac{\mathrm{d} u^{j}}{\mathrm{~d} s}=\frac{\operatorname{Grad} A}{|\operatorname{Grad} A|^{2}}(\mathbf{u})
$$

where Grad $A=g^{\ell k} A_{\mid \ell} \mathbf{x}_{\mid k}$ is the surface gradient and $g^{\ell k} g_{j k}=\delta_{j \ell}$. Since (B.1) implies on $\Gamma$ that

$$
A A_{\mid i}=(\mathbf{x}-\stackrel{\circ}{\mathbf{x}}) \cdot \mathbf{x}_{\mid i}=F_{i}=\mathbf{F} \cdot \mathbf{x}_{\mid i} \text { and } \operatorname{Grad} A=g^{\ell k} \frac{F_{\ell}}{A} \mathbf{x}_{\mid k}=\frac{1}{A} F^{k} \mathbf{x}_{\mid k}=\frac{1}{A} \mathbf{F}
$$

we find

$$
\operatorname{Grad} A \cdot \operatorname{Grad} A=\frac{1}{A^{2}} \mathbf{F} \cdot \mathbf{F}=\frac{1}{A^{2}}\left(A^{2}-G^{2}\right)=1-\frac{G^{2}}{A^{2}}
$$

and the differential equations (B.2) take the form

$$
\left.\frac{\mathrm{d} \mathbf{x}}{\mathrm{d} s}\right|_{\mathbf{c}_{\Theta}}=\mathbf{x}_{\mid j} \frac{\mathrm{d} u^{j}}{\mathrm{~d} s}=\frac{\operatorname{Grad} A}{|\mathbf{G r a d} A|^{2}}\left(1-\frac{G^{2}}{A^{2}}(\mathbf{u})\right)^{-1} .
$$

After multiplication with $\mathbf{x}_{\mid k} g^{\ell k}$, we thus arrive at

$$
\frac{\mathrm{d} u^{\ell}}{\mathrm{d} s}=\frac{1}{A(\mathbf{u})}\left(1-\frac{G^{2}}{A^{2}}(\mathbf{u})\right)^{-1} F^{\ell}(\mathbf{u}), F^{\ell}=g^{\ell k} \mathbf{F} \cdot \mathbf{x}_{\mid k}, \ell, k=1, \ldots, n-1
$$

Theorem B.1. To every $\stackrel{\circ}{\mathbf{x}} \in \Gamma$ there exists a neighborhood of $\stackrel{\circ}{\mathbf{x}}$ on $\Gamma$ where the system (B.4) admits a unique solution

$$
u^{\ell}(s, \boldsymbol{\Theta})=s \Theta^{\ell}+\mathcal{O}\left(s^{2}\right)
$$


for $s \geq 0$ which is the solution of the Volterra integral equations

$$
u^{\ell}(s, \boldsymbol{\Theta})=\stackrel{\circ}{u}^{\ell}+s \Theta^{\ell}+\int_{0}^{s}\left\{\frac{F^{\ell}(\mathbf{u}(\sigma, \boldsymbol{\Theta}))}{A(\mathbf{u}(\sigma, \boldsymbol{\Theta}))}\left(1-\frac{G^{2}(\mathbf{u}(\sigma, \boldsymbol{\Theta}))}{A^{2}(\mathbf{u}(\sigma, \boldsymbol{\Theta}))}\right)^{-1}-\Theta^{\ell}\right\} \mathrm{d} \sigma .
$$

The transformation $\mathbf{u} \mapsto(\varrho, \boldsymbol{\Theta})$ to surface polar coordinates about $\stackrel{\circ}{\mathbf{x}}$ is given by $\mathbf{u}(\varrho, \boldsymbol{\Theta})$, i.e., $s=\varrho \geq 0$.

Proof. Since

$$
\mathbf{F}=(\mathbf{x}-\stackrel{\circ}{\mathbf{x}})-((\mathbf{x}-\stackrel{\circ}{\mathbf{x}}) \cdot \mathbf{n}) \mathbf{n}
$$

the expansion about $\stackrel{\circ}{\mathbf{x}}=\mathbf{x}(\stackrel{\circ}{\mathbf{u}})$ gives on the one hand

$$
\begin{aligned}
& \mathbf{x}-\stackrel{\circ}{\mathbf{x}}=\stackrel{\circ}{\mathbf{x}}_{\mid k} \frac{\mathrm{d} \stackrel{\circ}{u}^{k}}{\mathrm{~d} s} s+\frac{1}{2}\left\{\stackrel{\circ}{\mathbf{x}}_{|k| \ell} \frac{\mathrm{d} \stackrel{\circ}{u}^{k}}{\mathrm{~d} s} \frac{\mathrm{d} \stackrel{\circ}{u}^{\ell}}{\mathrm{d} s}+\stackrel{\circ}{\mathbf{x}}_{\mid k} \frac{\mathrm{d}^{2} \stackrel{\circ}{u}^{k}}{\mathrm{~d} s^{2}}\right\} s^{2}
\end{aligned}
$$

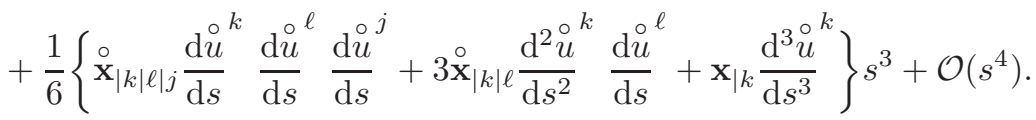

On the other hand, with the Gaussian equations

$$
\mathbf{x}_{|j| k}=\Gamma_{j k}^{\ell} \mathbf{x}_{\mid \ell}+L_{j k} \mathbf{n}, \ell=1, \ldots, n-1
$$

and the Weingarten relations

$$
\mathbf{n}_{\mid j}=-L_{j}^{m} \mathbf{x}_{\mid m}
$$

we obtain

$$
\mathbf{x}_{|j| k \mid \ell}=\left\{\Gamma_{j k \mid \ell}^{m}+\Gamma_{j k}^{r} \Gamma_{r \ell}^{m}-L_{j k} L_{\ell}^{m}\right\} \mathbf{x}_{\mid m}+\left\{L_{j k \mid \ell}+\Gamma_{j k}^{r} L_{r \ell}\right\} \mathbf{n}
$$

where $\Gamma_{t j}^{\ell}$ are the Christoffel symbols of the second kind of $\Gamma$ at $\mathbf{x}, \Gamma_{t j \mid k}^{\ell}=\frac{\partial}{\partial u^{k}} \Gamma_{t j}^{\ell}(\mathbf{u})$, and $L_{j}^{\ell}=g^{\ell k} L_{k j}$ with $L_{k j}$ the second fundamental form of $\Gamma$ at $\mathbf{x}$ (see e.g. [13, p. 90]). Here and in what follows, we abbreviate

$$
\stackrel{\circ}{\mathbf{x}}_{\mid k}=\mathbf{x}_{\mid k}(\stackrel{\circ}{\mathbf{u}}), \stackrel{\circ}{\mathbf{x}}_{|k| \ell}=\mathbf{x}_{|k| \ell}(\stackrel{\circ}{\mathbf{u}}) \text { and } \frac{\mathrm{d} \stackrel{\circ}{\mathrm{d} s}}{\mathrm{~d}}=\frac{\mathrm{d} u}{\mathrm{~d} s}(\stackrel{\circ}{u}) \text { etc. }
$$

We get thus from (B.6)

$$
\begin{aligned}
& \mathbf{x}-\stackrel{\circ}{\mathbf{x}}=\mathbf{e}(\boldsymbol{\Theta}) s+\frac{1}{2}\left\{\left(\stackrel{\circ}{\Gamma}_{k \ell}^{m} \stackrel{\circ}{\mathbf{x}}_{\mid m}+\stackrel{\circ}{L}_{k \ell} \stackrel{\circ}{\mathbf{n}}\right) \Theta^{k} \Theta^{\ell}+\stackrel{\circ}{\mathbf{x}}_{\mid k} \frac{\mathrm{d}^{2} \stackrel{\circ}{u}}{\mathrm{~d} s^{2}}\right\} s^{2} \\
& +\frac{1}{6}\left\{\left(\left[\stackrel{\circ}{\Gamma}_{j k \mid \ell}^{t}+\stackrel{\circ}{\Gamma}_{j k}^{m} \stackrel{\circ}{\Gamma}_{m \ell}^{t}-\stackrel{\circ}{L}_{j k} \stackrel{\circ}{L}_{\ell}^{t}\right] \stackrel{\circ}{\mathbf{x}}_{\mid t}+\left[\stackrel{\circ}{L}_{j k \mid \ell}+\stackrel{\circ}{\Gamma}_{j k}^{m} \stackrel{\circ}{L}_{m \ell}\right] \stackrel{\circ}{\mathbf{n}}\right) \Theta^{j} \Theta^{k} \Theta^{\ell}\right. \\
& \left.+3\left(\stackrel{\circ}{\Gamma}_{k \ell}^{t} \stackrel{\circ}{\mathbf{x}_{\mid t}}+\stackrel{\circ}{L}_{k \ell} \stackrel{\circ}{\mathbf{n}}\right) \Theta^{\ell} \frac{\mathrm{d}^{2} \stackrel{\circ}{u}^{k}}{\mathrm{~d} s^{2}}+\stackrel{\circ}{\mathbf{x}_{\mid t}} \frac{\mathrm{d}^{3} \stackrel{\circ}{u}^{t}}{\mathrm{~d} s^{3}}\right\} s^{3}+\mathcal{O}\left(s^{4}\right) .
\end{aligned}
$$

By combining this expansion with

$$
\mathbf{n}(\mathbf{u})=\stackrel{\circ}{\mathbf{n}}+\stackrel{\circ}{\mathbf{n}}_{\mid j} \frac{\mathrm{d} u}{\mathrm{~d} s} s+\frac{1}{2}\left\{\stackrel{\circ}{\mathbf{n}}_{|j| k} \Theta^{k} \Theta^{j}+\stackrel{\circ}{\mathbf{n}}_{\mid j} \frac{\mathrm{d}^{2} \stackrel{\circ}{ }^{j}}{\mathrm{~d} s^{2}}\right\} s^{2}+\mathcal{O}\left(s^{3}\right),
$$


we arrive at

$$
\begin{aligned}
& G=(\mathbf{x}-\stackrel{\circ}{\mathbf{x}}) \cdot \mathbf{n}(\mathbf{u})
\end{aligned}
$$

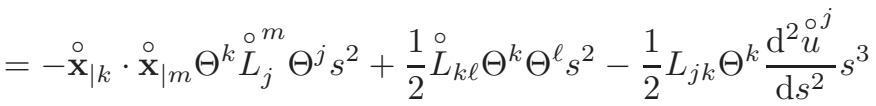

$$
\begin{aligned}
& +\left\{\frac{1}{6}\left(\stackrel{\circ}{L}_{j k \mid \ell}+\stackrel{\circ}{\Gamma}_{j k}^{m} \stackrel{\circ}{L}_{m \ell}\right)-\frac{1}{2}\left(\stackrel{\circ}{L}_{j m} \stackrel{\circ}{\Gamma}_{k \ell}^{m}+\stackrel{\circ}{L}_{j \mid k}^{\ell}+\stackrel{\circ}{L}_{j} \stackrel{\circ}{\Gamma}_{m k}^{\ell}\right)\right\} \Theta^{j} \Theta^{k} \Theta^{\ell} s^{3}+\mathcal{O}\left(s^{4}\right) \\
& =-\frac{1}{2} \stackrel{\circ}{L}_{k j} \Theta^{k} \Theta^{j} s^{2}+\left\{\frac{1}{6} \stackrel{\circ}{L}_{j k \mid \ell}-\frac{1}{3} \stackrel{\circ}{L}_{j m} \stackrel{\circ}{\Gamma}_{k \ell}^{m}-\frac{1}{2} \stackrel{\circ}{L}_{j \mid k}^{\ell}-\frac{1}{2} \stackrel{\circ}{L}_{j} \stackrel{\circ}{\Gamma}_{m k}^{\ell}\right\} \Theta^{j} \Theta^{k} \Theta^{\ell} s^{3} \\
& -\frac{1}{2} L_{j k} \Theta^{k} \frac{\mathrm{d}^{2} u^{j}}{\mathrm{~d} s^{2}} s^{3}+\mathcal{O}\left(s^{4}\right), \\
& \mathbf{F}=\mathbf{e}(\Theta) s+\left\{\frac{1}{2}\left(\left[\stackrel{\circ}{\Gamma}_{k \ell}^{m} \stackrel{\circ}{\mathbf{x}}_{\mid m}+\stackrel{\circ}{L}_{k \ell} \stackrel{\circ}{\mathbf{n}}\right] \Theta^{k} \Theta^{\ell}+\stackrel{\circ}{\mathbf{x}}_{\mid k} \frac{\mathrm{d}^{2} \stackrel{\circ}{u}}{\mathrm{~d} s^{2}}\right)+\frac{1}{2} \stackrel{\circ}{L}_{k \ell} \Theta^{k} \Theta^{\ell} \stackrel{\circ}{\mathbf{n}}\right\} s^{2}+\mathcal{O}\left(s^{3}\right) \\
& A=s+\mathcal{O}\left(s^{2}\right) \text {. }
\end{aligned}
$$

Hence, we conclude

$$
\frac{\mathbf{F}}{A}\left(1-\frac{G^{2}}{A^{2}}\right)^{-1}=\mathbf{e}(\boldsymbol{\Theta})+\mathcal{O}(s)
$$

for any $C^{2}$-curve $\mathbf{x}(\mathbf{u}(s))$ through $\stackrel{\circ}{\mathbf{x}}$. Consequently, the kernel function $\{\ldots\}$ of the Volterra operator in (B.5) is continuous and there exists a solution $\mathbf{u}(s, \boldsymbol{\Theta})$ for fixed given $\boldsymbol{\Theta}$ in some vicinity of $\stackrel{\circ}{\mathrm{x}}$ on $\Gamma$. This solution is in $C^{1}([0, S])$ for some $S>0$ and is as many times continuously differentiable for $s \geq 0$ as is the manifold $\Gamma$.

The equation (B.3) implies

$$
1=\operatorname{Grad} A \cdot \mathbf{x}_{\mid j} \frac{\mathrm{d} u^{j}}{\mathrm{~d} s}=g^{\ell k} A_{\mid k} \mathbf{x}_{\mid \ell} \cdot \mathbf{x}_{\mid j} \frac{\mathrm{d} u^{j}}{\mathrm{~d} s}=g^{\ell k} g_{\ell j} A_{\mid k} \frac{\mathrm{d} u^{j}}{\mathrm{~d} s}=A_{\mid j} \frac{\mathrm{d} u^{j}}{\mathrm{~d} s}=\frac{\mathrm{d} A}{\mathrm{~d} s}=\frac{\mathrm{d} \varrho}{\mathrm{d} s} .
$$

Thus, it holds $A=s=\varrho$ and $\mathbf{u}(\varrho, \boldsymbol{\Theta})$, the solution of (B.5), is the desired transformation $(\varrho, \Theta) \mapsto \mathbf{u}$

By bootstrapping, it follows from (B.4) that $\mathbf{u}(\varrho, \boldsymbol{\Theta})$ is higher order differentiable up to $\varrho=0$. To see this, consider the Taylor expansions of the left and the right hand sides of the equations (B.4) about $\stackrel{\circ}{\mathbf{u}}$ up to the order two:

Left hand side of (B.4): (by using (B.3))

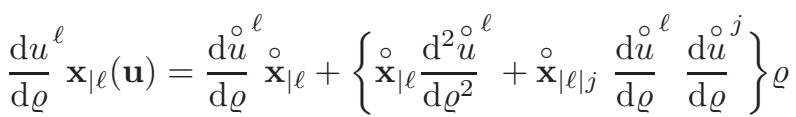

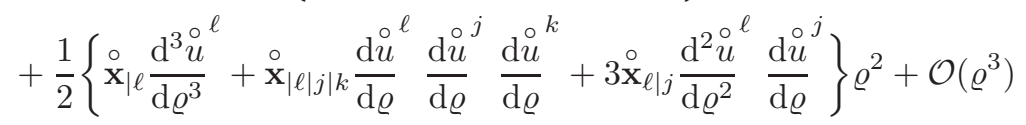

$$
\begin{aligned}
& =\stackrel{\circ}{\mathbf{x}}_{\mid \ell} \Theta^{\ell}+\left\{\stackrel{\circ}{\dot{x}_{\mid \ell}} \frac{\mathrm{d}^{2} \stackrel{\circ}{\ell}}{\mathrm{d} \varrho^{2}}+\left(\stackrel{\circ}{\Gamma}_{\ell j}^{m} \stackrel{\circ}{\mathbf{x}}_{\mid m}+\stackrel{\circ}{\Gamma}_{\ell j} \stackrel{\circ}{\mathbf{n}}\right) \Theta^{\ell} \Theta^{j}\right\} \varrho \\
& +\frac{1}{2}\left\{\stackrel{\circ}{\mathbf{x}}_{\ell \ell} \frac{\mathrm{d}^{3} \stackrel{\circ}{u}}{\mathrm{~d} \varrho^{3}}+\left(\left[\stackrel{\circ}{\Gamma}_{\ell j \mid k}+\stackrel{\circ}{\Gamma}_{\ell j}^{t} \stackrel{\circ}{\Gamma}_{t k}-\stackrel{\circ}{L}_{\ell j} \stackrel{\circ}{L}_{k}^{m}\right] \stackrel{\circ}{\mathbf{x}}_{\mid m}+\left[\stackrel{\circ}{\Gamma}_{\ell j} \stackrel{\circ}{L}_{m k}+\stackrel{\circ}{L}_{\ell j \mid k}\right] \stackrel{\circ}{\mathbf{n}}\right) \Theta^{\ell} \Theta^{j} \Theta^{k}\right. \\
& \left.+3\left(\stackrel{\circ}{\Gamma}_{\ell j}^{m} \stackrel{\circ}{\mathbf{x}}_{\mid m}+\stackrel{\circ}{L}_{\ell j} \stackrel{\circ}{\mathbf{n}}\right) \Theta^{j} \frac{\mathrm{d}^{2} \stackrel{\circ}{u}^{\ell}}{\mathrm{d} \varrho^{2}}\right\} \varrho^{2}+\mathcal{O}\left(\varrho^{3}\right) \text {. }
\end{aligned}
$$




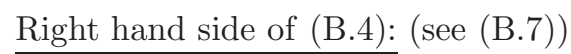

$$
\begin{aligned}
& \frac{1}{\varrho}(\mathbf{x}-\stackrel{\circ}{\mathbf{x}})=\stackrel{\circ}{\mathbf{x}}_{\mid \ell} \Theta^{\ell}+\frac{1}{2}\left\{\left(\stackrel{\circ}{\Gamma}_{k \ell}^{m}{\stackrel{\circ}{\mathbf{x}_{\mid m}}}_{\mid}+\stackrel{\circ}{L}_{k \ell} \stackrel{\circ}{\mathbf{n}}\right) \Theta^{k} \Theta^{\ell}+\stackrel{\circ}{\mathbf{x}}_{\mid k} \frac{\mathrm{d}^{2} \stackrel{\circ}{u}}{\mathrm{~d} \varrho^{2}}\right\} \varrho \\
& +\frac{1}{6}\left\{\stackrel{\circ}{\mathbf{x}_{\mid \ell}} \frac{\mathrm{d}^{3} \stackrel{\circ}{u}^{\ell}}{\mathrm{d} \varrho^{3}}+\left(\left[\stackrel{\circ}{\Gamma}_{\ell j \mid k}^{m}+\stackrel{\circ}{\Gamma}_{\ell j}^{t} \stackrel{\circ}{\Gamma}_{t k}^{m}-\stackrel{\circ}{L}_{\ell j} \stackrel{\circ}{L}_{k}^{m}\right] \stackrel{\circ}{\mathbf{x}}_{\mid m}+\left[\stackrel{\circ}{\Gamma}_{\ell j}^{m} \stackrel{\circ}{L}_{m k}+\stackrel{\circ}{L}_{\ell j \mid k}\right] \stackrel{\circ}{\mathbf{n}}\right) \Theta^{\ell} \Theta^{j} \Theta^{k}\right. \\
& \left.+3\left(\stackrel{\circ}{\Gamma}_{\ell j}^{m} \stackrel{\circ}{\mathbf{x}}_{\mid m}+\stackrel{\circ}{L}_{\ell j} \stackrel{\circ}{\mathbf{n}}\right) \Theta^{j} \frac{\mathrm{d}^{2} \stackrel{\circ}{u}}{\mathrm{~d} \varrho^{2}}\right\} \varrho^{2}+\mathcal{O}\left(\varrho^{3}\right), \\
& \mathbf{n}(\mathbf{u})=\stackrel{\circ}{\mathbf{n}}+\stackrel{\circ}{\mathbf{n}}_{\mid j} \frac{\mathrm{d} \stackrel{\circ}{u}^{j}}{\mathrm{~d} \varrho} \varrho+\frac{1}{2}\left\{\stackrel{\circ}{\mathbf{n}}_{|j| k} \frac{\mathrm{d} \stackrel{\circ}{j}^{j} \varrho}{\mathrm{d} \varrho} \frac{\stackrel{\circ}{u}^{k}}{\mathrm{~d} \varrho}+\stackrel{\circ}{\mathbf{n}}_{\mid j} \frac{\mathrm{d}^{2} \stackrel{\circ}{u}^{j}}{\mathrm{~d} \varrho^{2}}\right\} \varrho^{2}+\mathcal{O}\left(\varrho^{3}\right) \\
& =\stackrel{\circ}{\mathbf{n}}-\stackrel{\circ}{L}_{j} \stackrel{\circ}{\mathbf{x}}_{\mid m} \Theta^{j} \varrho \\
& -\frac{1}{2}\left\{\left(\stackrel{\circ}{L}_{j \mid k} \stackrel{\circ}{\mathbf{x}_{\mid m}}+\stackrel{\circ}{L}_{j}^{\ell} \stackrel{\circ}{\Gamma}_{\ell k} \stackrel{\circ}{\mathbf{x}}_{\mid m}+\stackrel{\circ}{L}_{j} \stackrel{\circ}{L}_{m k} \stackrel{\circ}{\mathbf{n}}\right) \Theta^{j} \Theta^{k}+\stackrel{\circ}{L}_{j} \frac{\mathrm{d}^{2} \stackrel{\circ}{u}^{j}}{\mathrm{~d}^{2}} \stackrel{\circ}{\mathbf{x}}_{\mid m}\right\} \varrho^{2}+\mathcal{O}\left(\varrho^{3}\right), \\
& \frac{1}{\varrho} G=\frac{1}{2} \stackrel{\circ}{L}_{k \ell} \Theta^{k} \Theta^{\ell} \varrho-\frac{1}{2} \stackrel{\circ}{L}_{j \ell} \frac{\mathrm{d}^{2} \stackrel{\circ}{\mathrm{d} \varrho^{2}}}{\mathrm{\Theta}^{\ell}} \\
& +\left\{\frac{1}{6} \stackrel{\circ}{L}_{\ell j \mid k}-\frac{1}{3} \stackrel{\circ}{\Gamma}_{\ell j}^{m} \stackrel{\circ}{L}_{m k}-\frac{1}{2} \stackrel{\circ}{g}_{m \ell} \stackrel{\circ}{L_{j \mid k}^{m}}-\frac{1}{2} \stackrel{\circ}{g}_{m \ell} \stackrel{\circ}{L}_{j} \stackrel{\circ}{\Gamma}_{t k}^{m}\right\} \Theta^{j} \Theta^{k} \Theta^{\ell} \varrho^{2}+\mathcal{O}\left(\varrho^{3}\right), \\
& \left(\frac{1}{\varrho} G\right) \mathbf{n}(\mathbf{u})=\left(\frac{1}{\varrho} G\right)\left(\stackrel{\circ}{\mathbf{n}}-\stackrel{\circ}{L}_{j} \Theta^{j} \mathbf{x}_{\mid m} \varrho\right)+\mathcal{O}\left(\varrho^{3}\right) \\
& =\stackrel{\circ}{\mathbf{n}} \frac{\stackrel{\circ}{2}}{L} k \ell \Theta^{k} \Theta^{\ell} \varrho+\stackrel{\circ}{\mathbf{n}} \varrho^{2}-\frac{1}{2} \stackrel{\circ}{L}_{j} \stackrel{\circ}{L}_{k \ell} \stackrel{\circ}{\mathbf{x}_{\mid m}} \Theta^{j} \Theta^{k} \Theta^{\ell} \varrho^{2}-\frac{1}{2} \stackrel{\circ}{L}_{j \ell} \frac{\mathrm{d}^{2} \stackrel{\circ}{u}}{\mathrm{~d} \varrho^{2}} \Theta^{\ell} \stackrel{\circ}{\mathbf{n}} \varrho^{2}+\mathcal{O}\left(\varrho^{3}\right), \\
& \frac{G^{2}}{A^{2}}=\left(\frac{1}{\varrho} G\right)^{2}=\frac{1}{4}\left(\stackrel{\circ}{L}_{k \ell} \Theta^{k} \Theta^{\ell}\right)^{2} \varrho^{2}+\mathcal{O}\left(\varrho^{3}\right), \\
& \left(1-\frac{G^{2}}{A^{2}}\right)^{-1}=1+\frac{1}{4}\left(\stackrel{\circ}{L}_{k \ell} \Theta^{k} \Theta^{\ell}\right)^{2} \varrho^{2}+\mathcal{O}\left(\varrho^{3}\right) \text {. }
\end{aligned}
$$

Comparing the coefficients of $\mathbf{x}_{\mid \ell \varrho}$ gives:

$$
\underline{\text { lhs: }} \quad \frac{\mathrm{d}^{2} \stackrel{\circ}{\ell}^{\ell}}{\mathrm{d} \varrho^{2}}+\stackrel{\circ \ell}{\Gamma}{ }_{j k} \Theta^{j} \Theta^{k} \quad \text { and } \quad \underline{\text { rhs: }} \quad \frac{1}{2} \stackrel{\circ}{\Gamma}_{j k}^{\ell} \Theta^{j} \Theta^{k}+\frac{1}{2} \frac{\mathrm{d}^{2} \stackrel{\circ}{u}^{\ell}}{\mathrm{d} \varrho^{2}} \text {. }
$$

Consequently, there holds

$$
\frac{\mathrm{d}^{2} \stackrel{\circ}{\ell}}{\mathrm{d} \varrho^{2}}(\stackrel{\circ}{\mathbf{u}})=-\stackrel{\circ}{\Gamma}_{j k}^{\ell} \Theta^{j} \Theta^{k}
$$

Next, compare the coefficients of $\stackrel{\circ}{x}_{\mid \ell} \varrho^{2}$ :

$\underline{\text { Left hand side of (B.4): }}$

$$
\begin{aligned}
& \frac{1}{2} \frac{\mathrm{d}^{3} \stackrel{\circ}{\mathrm{d} \varrho^{3}}}{\mathrm{~d}}+\frac{1}{2}\left(\stackrel{\circ}{\Gamma}_{m j \mid k}^{\ell}+\stackrel{\circ}{\Gamma}_{m j}^{t} \stackrel{\circ}{\Gamma}_{t k}^{\ell}-\stackrel{\circ}{L}_{m j} \stackrel{\circ}{L_{k}^{\ell}}\right) \Theta^{m} \Theta^{j} \Theta^{k}+\frac{3}{2} \stackrel{\circ}{\Gamma}_{m j}^{\ell} \Theta^{j} \frac{\mathrm{d}^{2} \stackrel{\circ}{\mathrm{d} \varrho^{2}}}{} \\
& \quad=\frac{1}{2} \frac{\mathrm{d}^{3} \stackrel{\circ}{u}}{\mathrm{~d} \varrho^{3}}+\frac{1}{2}\left(\stackrel{\circ}{\Gamma}_{m j \mid k}^{\ell}+\stackrel{\circ}{\Gamma}_{m j}^{t} \stackrel{\circ}{\Gamma}_{t k}^{\ell}-\stackrel{\circ}{L}_{m j} \stackrel{\circ}{L}_{k}^{\ell}\right) \Theta^{m} \Theta^{j} \Theta^{k}-\frac{3}{2} \stackrel{\circ}{\Gamma}_{t j} \stackrel{\circ}{\Gamma}_{k m}^{t} \Theta^{m} \Theta^{j} \Theta^{k}
\end{aligned}
$$


Right hand side of (B.4):

$$
\begin{aligned}
& \left(\frac{1}{\varrho}(\mathbf{x}-\stackrel{\circ}{\mathbf{x}})-\frac{1}{\varrho} G \mathbf{n}\right)\left(1+\frac{1}{4} \varrho^{2}\left(\stackrel{\circ}{L} k \ell \Theta^{k} \Theta^{\ell}\right)^{2}+\mathcal{O}\left(\varrho^{3}\right)\right) \\
& =\Theta^{\ell} \stackrel{\circ}{\mathbf{x}}_{\mid \ell}-\varrho \frac{1}{2} \stackrel{\circ}{\Gamma}_{k m}^{\ell} \Theta^{k} \Theta^{m} \stackrel{\circ}{\mathbf{x}}_{\mid \ell} \\
& +\left\{\frac{1}{6} \frac{\mathrm{d}^{3} \stackrel{\circ}{u}}{\mathrm{~d} \varrho^{3}}+\left(\frac{1}{6} \stackrel{\circ}{\Gamma}_{m j \mid k}-\frac{1}{3} \stackrel{\circ}{\Gamma}_{m j} \stackrel{\circ \ell}{\Gamma}{ }_{t k}-\frac{1}{3} \stackrel{\circ}{L}_{j}^{\ell} \stackrel{\circ}{L m}_{k m}\right) \Theta^{m} \Theta^{j} \Theta^{k}+\frac{1}{4}\left(\stackrel{\circ}{L}_{k j} \Theta^{k} \Theta^{j}\right)^{2} \Theta^{\ell}\right\} \stackrel{\circ}{\mathbf{x}_{\mid \ell}}
\end{aligned}
$$

Hence, from equating left hand side and right side, one obtains

$$
\frac{\mathrm{d}^{3} u^{\ell}}{\mathrm{d} \varrho^{3}}(\stackrel{\circ}{\mathbf{u}})=\left\{-\stackrel{\circ \ell}{\Gamma_{m j \mid k}}+2 \stackrel{\circ}{\Gamma}_{m j} \stackrel{\circ \ell}{\Gamma_{t k}}+\frac{1}{2} \stackrel{\circ}{L}_{m j} \stackrel{\circ}{L}{ }_{k}^{\ell}\right\} \Theta^{m} \Theta^{j} \Theta^{k}+\frac{3}{4}\left(\stackrel{\circ}{L}_{k j} \Theta^{k} \Theta^{j}\right)^{2} \Theta^{\ell}
$$
(B.6):

With the expressions for $\frac{\mathrm{d}^{2} u^{\ell}}{\mathrm{d} s^{2}}$ and $\frac{\mathrm{d}^{3} u^{\ell}}{\mathrm{d} s^{3}}$, we recollect the relations for $\mathbf{x}-\stackrel{\circ}{\mathbf{x}}$ and find instead of

$$
\begin{aligned}
& \left(\mathbf{x}-\mathbf{x}_{0}\right)=\varrho \mathbf{e}(\boldsymbol{\Theta})+\frac{1}{2} \varrho^{2} \stackrel{\circ}{\mathbf{n}} \stackrel{\circ}{L} \ell \Theta^{k} \Theta^{\ell} \\
& +\frac{\varrho^{3}}{6}\left\{\left(-\frac{1}{2} \stackrel{\circ}{\mathbf{x}}_{\mid \ell} \stackrel{\circ}{L}_{m j} \stackrel{\circ}{L}_{k}^{\ell}+\left[2 \stackrel{\circ}{L}_{m j \mid k}-3 \stackrel{\circ}{L}_{t j} \stackrel{\circ}{\Gamma}_{m k}^{t}\right] \stackrel{\circ}{\mathbf{n}}\right) \Theta^{m} \Theta^{j} \Theta^{k}+\frac{4}{3}\left(\stackrel{\circ}{L}_{k j} \Theta^{k} \Theta^{j}\right)^{2} \boldsymbol{\Theta}\right\}+\mathcal{O}\left(\varrho^{4}\right) .
\end{aligned}
$$

Collecting the first three derivatives of $u^{\ell}$ at $\stackrel{\circ}{\mathbf{u}}$ implies that the first terms of the transform $(\varrho, \boldsymbol{\Theta}) \mapsto \mathbf{u}$ read as

$$
\begin{aligned}
& u^{\ell}(\varrho, \Theta)=\stackrel{\circ}{u}+\Theta^{\ell} \varrho-\frac{1}{2} \stackrel{\circ}{\Gamma}_{j k}^{\ell} \Theta^{j} \Theta^{k} \varrho^{2} \\
& \quad+\frac{1}{6}\left\{\frac{1}{2} \stackrel{\circ}{L}_{j}^{\ell} \stackrel{\circ}{L}_{k m}+2 \stackrel{\circ \ell}{\Gamma_{t j}} \stackrel{\circ}{\Gamma}_{k m}^{t}-\stackrel{\circ}{\Gamma}_{m j \mid k}^{\ell}\right\} \Theta^{j} \Theta^{k} \Theta^{m} \varrho^{3}+\frac{1}{8}\left(\stackrel{\circ}{L}_{j k} \Theta^{j} \Theta^{k}\right)^{2} \Theta^{\ell} \varrho^{3}+\mathcal{O}\left(\varrho^{4}\right),
\end{aligned}
$$

for all $\ell=1, \ldots, n-1$. Whereas, the inverse mapping $\mathbf{u} \mapsto(\varrho, \Theta)$ can be obtained from

$$
\varrho=A(\mathbf{u})=|\mathbf{x}(\mathbf{u})-\stackrel{\circ}{\mathbf{x}}|
$$

and the nonlinear equations for

$$
\begin{aligned}
\Theta^{\ell} & =\frac{1}{\varrho}\left(u^{\ell}-\stackrel{\circ}{\ell}\right)+\frac{1}{2} \varrho \stackrel{\circ}{\Gamma}_{j k}^{\ell} \Theta^{j} \Theta^{k} \\
& -\frac{1}{6} \varrho^{2}\left\{\frac{1}{2} \stackrel{\circ}{L}_{j}^{\ell} L_{k m}+2 \stackrel{\circ}{\Gamma_{t j}^{\ell}} \stackrel{\circ}{\Gamma}_{k m}^{t}-\stackrel{\circ}{\Gamma}_{m j \mid k}^{\ell}\right\} \Theta^{j} \Theta^{k} \Theta^{m}-\frac{1}{8} \varrho^{2}\left(\stackrel{\circ}{L}_{j k} \Theta^{j} \Theta^{k}\right)^{2} \Theta^{\ell}+\ldots
\end{aligned}
$$

for all $\ell=1, \ldots, n-1$ can, for $\varrho>0$ sufficiently small, be solved via successive iteration.

Theorem B.2. Let $\Gamma \in C^{4}$. Then, the surface measure $\mathrm{d} s_{\Gamma}$ of $\Gamma$ in Martensen's surface polar coordinates satisfies

$$
\mathrm{d} s_{\Gamma}=\varrho^{n-2} \mathrm{~d} \varrho \wedge \mathrm{d} \boldsymbol{\omega}+\left(\varrho^{n} a(\boldsymbol{\Theta})+\mathcal{O}\left(\varrho^{n+1}\right)\right) \mathrm{d} \varrho \wedge \mathrm{d} \boldsymbol{\omega}
$$

where

$$
a(\Theta)=\sum_{j=1}^{n-1} \Theta^{j}\left\{\frac{3}{4}\left(\left(\stackrel{\circ}{L}_{k t} \Theta^{k} \Theta^{t}\right)^{2}+\stackrel{\circ}{L_{t}} \stackrel{\circ}{L_{m k}} \Theta^{t} \Theta^{m} \Theta^{k}\right)-\Theta^{j} \stackrel{\circ}{L}_{m}^{\ell} \stackrel{\circ}{L}_{\ell k} \Theta^{m} \Theta^{k}\right\}
$$

and

$$
\mathrm{d} \boldsymbol{\omega}=\sum_{j=1}^{n-1}(-1)^{j+1} \Theta^{j}\left[\mathrm{~d} \Theta^{1} \wedge \cdots \wedge \mathrm{d} \Theta^{j} \wedge \cdots \wedge \mathrm{d} \Theta^{n-1}\right], \sum_{j=1}^{n-1} \Theta^{j} \Theta^{j}=1 .
$$

Here, $\mathrm{d} \boldsymbol{\omega}$ is the surface measure of the unit sphere $\mathbb{S}^{n-2}$ in $\mathbb{R}^{n-1}$. 
Proof. For the surface measure of $\Gamma$, we use the exterior Pfaffian products, defining the exterior normal vector's components times $\mathrm{d} s_{\Gamma}$ (see [21, Chapter 11.4]. Then, we multiply scalarly with the exterior unit normal $\mathbf{n}(\mathbf{x})=\left(n^{1}(\mathbf{x}), \ldots, n^{n}(\mathbf{x})\right)$ which yields

$$
\mathrm{d} s_{\Gamma}=\sum_{j=1}^{n}(-1)^{j+1}\left[\mathrm{~d} x^{1} \wedge \cdots \wedge \mathrm{d} / x^{j} \wedge \cdots \wedge \mathrm{d} x^{n}\right] n^{j}(\mathbf{x}) .
$$

Expansions about $\stackrel{\circ}{\mathbf{x}}$ up to the order $\varrho^{2}$ give

$$
\begin{aligned}
& \mathbf{n}(\mathbf{x})=\stackrel{\circ}{\mathbf{n}}-\varrho \stackrel{\circ}{L}_{j}^{m} \stackrel{\circ}{\mathbf{x}}_{\mid m} \Theta^{j}-\frac{1}{2} \varrho^{2}\left\{\begin{array}{l}
\stackrel{\circ}{L} \\
L_{j \mid k}+\stackrel{\circ}{L}_{j} \stackrel{\circ}{\Gamma}_{\ell k}
\end{array}-\stackrel{\circ}{\Gamma}_{j k}^{t} \stackrel{\circ}{L}_{t}^{m}\right\} \Theta^{j} \Theta^{k} \stackrel{\circ}{\mathbf{X}}_{\mid m} \\
& -\frac{1}{2} \varrho^{2} \stackrel{\circ}{L_{j}^{\ell}} \stackrel{\circ}{L k}_{\ell k} \Theta^{j} \Theta^{k} \stackrel{\circ}{\mathbf{n}}+\mathcal{O}\left(\varrho^{3}\right)
\end{aligned}
$$

and

$$
\begin{aligned}
\mathrm{d} x^{j} & =\mathrm{d} \varrho \Theta^{j}+\varrho \mathrm{d} \Theta^{j}+\frac{1}{2} \varrho^{2} \mathrm{~d} \varrho\left\{\frac{3}{4}\left(\stackrel{\circ}{L}_{k t} \Theta^{k} \Theta^{t}\right)^{2}-\frac{1}{2} \stackrel{\circ}{L}_{k}^{j} \stackrel{\circ}{m}_{m t} \Theta^{k} \Theta^{m} \Theta^{t}\right\}+\mathcal{O}\left(\varrho^{3}\right) \\
& =\mathrm{d} \varrho \Theta^{j}+\varrho \Theta^{j}+\varrho^{2} \mathrm{~d} \varrho c^{j}(\Theta)+\mathcal{O}\left(\varrho^{3}\right)
\end{aligned}
$$

with

$$
c^{j}(\Theta)=\frac{1}{2}\left\{\frac{3}{4}\left(\stackrel{\circ}{L}_{k t} \Theta^{k} \Theta^{t}\right)^{2}-\frac{1}{2} \stackrel{\circ}{L}_{k}^{j} \stackrel{\circ}{L}_{m t} \Theta^{k} \Theta^{m} \Theta^{t}\right\}
$$

for $j=1, \ldots, n-1$ and

$$
\mathrm{d} x^{n}=\stackrel{\circ}{L}_{j k} \Theta^{j} \Theta^{k} \varrho \mathrm{d} \varrho+\frac{1}{2}\left(2 \stackrel{\circ}{L}_{m j \mid k}-3 \stackrel{\circ}{L}_{\ell j} \stackrel{\circ}{\Gamma}_{m k}\right) \Theta^{m} \Theta^{j} \Theta^{k} \varrho^{2} \mathrm{~d} \varrho+\mathcal{O}\left(\varrho^{3}\right) .
$$

Inserting this into (B.10), yields

$$
\begin{aligned}
\mathrm{d} s_{\Gamma}= & (-1)^{n+1}\left[\mathrm{~d} x^{1} \wedge \cdots \wedge \mathrm{d} x^{n-1}\right]\left(1-\frac{1}{2} \varrho^{2} \stackrel{\circ}{L}_{j} \stackrel{\circ}{L}_{m k} \Theta^{j} \Theta^{k}+\mathcal{O}\left(\varrho^{3}\right)\right) \\
+ & \sum_{j=1}^{n-1}\left[\left[\mathrm{~d} x^{1} \wedge \cdots \wedge \mathrm{d} / x^{j} \wedge \mathrm{d} x^{n-1}\right] \wedge \mathrm{d} x^{n}\right] \\
& \times\left\{-\varrho^{\circ} L_{k}^{j} \Theta^{k}-\frac{1}{2}\left(\stackrel{\circ}{L}_{m \mid k}^{j}+\stackrel{\circ}{L}_{m}^{\ell} \stackrel{\circ}{\Gamma}_{\ell k}^{j}-\stackrel{\circ}{\Gamma}_{m k}^{t} \stackrel{\circ}{L}_{t}^{j}\right) \Theta^{m} \Theta^{k} \varrho^{2}+\mathcal{O}\left(\varrho^{3}\right)\right\} .
\end{aligned}
$$

For the first term in (B.11), we obtain (modulo $\mathcal{O}\left(\varrho^{3}\right)$ terms) with the relations for $\mathrm{d} x^{j}$ :

$$
\begin{aligned}
& {\left[\mathrm{d} x^{1} \wedge \cdots \wedge \mathrm{d} x^{n-1}\right]=\left[\mathrm{d} \varrho \Theta^{1}+\varrho \mathrm{d} \Theta^{1}+\varrho^{2} \mathrm{~d} \varrho c^{1}(\boldsymbol{\Theta}) \wedge \mathrm{d} \varrho \Theta^{2}+\varrho \mathrm{d} \Theta^{2}+\varrho^{2} \mathrm{~d} \varrho c^{2}(\boldsymbol{\Theta})\right.} \\
& \left.\wedge \cdots \wedge \mathrm{d} \varrho \Theta^{n-1}+\varrho \mathrm{d} \Theta^{n-1}+\varrho^{2} \mathrm{~d} \varrho c^{n-1}(\boldsymbol{\Theta})\right]=\varrho^{n-1}\left[\mathrm{~d} \Theta^{1} \wedge \cdots \wedge \mathrm{d} \Theta^{n-1}\right] \\
& +\varrho^{n-2}\left[\mathrm{~d} \varrho \wedge \sum_{j=1}^{n-1}(-1)^{j}\left\{\Theta^{j}+\varrho^{2} c^{j}(\boldsymbol{\Theta})\right\}\left[\mathrm{d} \Theta^{1} \wedge \cdots \wedge \mathrm{d} \Theta^{j} \wedge \cdots \wedge \mathrm{d} \Theta^{n-1}\right]\right] .
\end{aligned}
$$

Since the variables $\Theta^{j}$ vary on the $(n-2)$-dimensional sphere $\mathbb{S}^{n-2}$, where

$$
\sum_{j=1}^{n-1}\left(\Theta^{j}\right)^{2}=1
$$

we have

$$
\sum_{j=1}^{n-1} \Theta^{j} \mathrm{~d} \Theta^{j}=0
$$

on $\mathbb{S}^{n-2}$. Hence, the differentials $\mathrm{d} \Theta^{j}$ with $j=1, \ldots, n-1$ are linearly dependent and

$$
\left[\mathrm{d} \Theta^{1} \wedge \cdots \wedge \mathrm{d} \Theta^{n-1}\right]=0 .
$$


Moreover, on $\mathbb{S}^{n-2}$, we have that the exterior unit normal vector $\boldsymbol{\nu}$ to $\mathbb{S}^{n-2}$ satisfies

$$
\boldsymbol{\nu}_{\boldsymbol{\omega}}=\sum_{j=1}^{n-1} \Theta^{j} \mathbf{e}_{j}
$$

Therefore, it holds

$$
\nu_{j} \mathrm{~d} \boldsymbol{\omega}=(-1)^{j+1}\left[\mathrm{~d} \Theta^{1} \wedge \cdots \wedge \mathrm{d}\left(\Theta^{j} \wedge \cdots \wedge \mathrm{d} \Theta^{n-1}\right]=\Theta^{j} \mathrm{~d} \boldsymbol{\omega},\right.
$$

and with (B.12) one obtains

$$
\mathrm{d} \boldsymbol{\omega}=\sum_{j=1}^{n-1}(-1)^{j+1} \Theta^{j}\left[\mathrm{~d} \Theta^{1} \wedge \cdots \wedge d \Theta^{j} \wedge \cdots \wedge \mathrm{d} \Theta^{n-1}\right] .
$$

For the first term in (B.11), we find therefore

$$
\mathrm{d} s_{\Gamma}^{1}=(-1)^{n+1}\left\{\varrho^{n-2}+\varrho^{n}\left(\sum_{j=1}^{n-1} c^{j}(\boldsymbol{\Theta}) \Theta^{j}-\frac{1}{2} \stackrel{\circ}{L}_{m} \stackrel{\circ}{L}_{\ell k} \Theta^{m} \Theta^{k}\right)+\mathcal{O}\left(\varrho^{n+1}\right)\right\} \mathrm{d} \varrho \wedge \mathrm{d} \boldsymbol{\omega} .
$$

For the remaining terms in (B.11), we have (modulo $\mathcal{O}\left(\varrho^{3}\right)$ terms) that

$$
\begin{aligned}
& \mathrm{d} s_{\Gamma}^{2}=\left[\left[\mathrm{d} x^{1} \wedge \cdots \wedge \mathcal{W} / x^{j} \wedge \cdots \wedge \mathrm{d} x^{n-1}\right] \wedge \mathrm{d} x^{n}\right]\left(-\varrho L_{t}^{j} \Theta^{t}-\frac{1}{2} \varrho^{2}\{\ldots\} \Theta^{m} \Theta^{k}\right) \\
& =\left[\left[\mathrm{d} \varrho \Theta^{1}+\varrho \mathrm{d} \Theta^{1}+\varrho^{2} \mathrm{~d} \varrho c^{1}(\boldsymbol{\Theta}) \wedge \mathrm{d} \varrho \Theta^{2}+\varrho \mathrm{d} \Theta^{2}+\varrho^{2} \mathrm{~d} \varrho c^{2}(\boldsymbol{\Theta})\right.\right. \\
& \left.\wedge \cdots \wedge \downarrow / x^{j} \wedge \cdots \wedge \mathrm{d} \varrho^{2} \Theta^{n-1}+\varrho \mathrm{d} \Theta^{n-1}+\varrho^{2} \mathrm{~d} \varrho c^{n-1}(\Theta)\right] \\
& \left.\wedge\left(\varrho \mathrm{d} \varrho \stackrel{\circ}{j k}_{j k} \Theta^{j} \Theta^{k}+\frac{1}{2} \varrho^{2} \mathrm{~d} \varrho\{\ldots\} \Theta^{m} \Theta^{j} \Theta^{k}\right)\right]\left(-\varrho L_{t}^{k} \Theta^{t}-\frac{1}{2}\{\ldots\} \Theta^{m} \Theta^{k}\right) \\
& =\sum_{j=1}^{n-1}(-1)^{n+j}\left(\varrho^{n}+\mathcal{O}\left(\varrho^{n+1}\right)\right) \mathrm{d} \varrho \wedge\left[\mathrm{d} \Theta^{1} \wedge \cdots \wedge \mathrm{d}\left(\Theta^{j} \wedge \cdots \wedge \mathrm{d} \Theta^{n-1}\right] \stackrel{\circ}{L} m k \Theta^{m} \Theta^{k} \stackrel{\circ}{L}_{t}^{j} \Theta^{t}\right. \\
& =(-1)^{n+1} \sum_{j=1}^{n-1} \stackrel{\circ}{L}_{m k} \Theta^{m} \Theta^{k} \stackrel{\circ}{L}_{t}^{j} \Theta^{t} \Theta^{j}\left(\varrho^{n}+\mathcal{O}\left(\varrho^{n+1}\right)\right) \mathrm{d} \varrho \wedge \mathrm{d} \boldsymbol{\omega} .
\end{aligned}
$$

Consequently, we finally get in (B.11)

$$
\mathrm{d} s_{\Gamma}=\mathrm{d} s_{\Gamma}^{1}+\mathrm{d} s_{\Gamma}^{2}=\varrho^{n-2} \mathrm{~d} \varrho \wedge \mathrm{d} \boldsymbol{\omega}+\left(\varrho^{n} a(\boldsymbol{\Theta})+\mathcal{O}\left(\varrho^{n+1}\right)\right) \mathrm{d} \varrho \wedge \mathrm{d} \boldsymbol{\omega}
$$

with

$$
a(\Theta)=\sum_{j=1}^{n-1} \Theta^{j}\left\{\frac{3}{4}\left(\stackrel{\circ}{L}_{k t} \Theta^{k} \Theta^{t}\right)^{2}+\stackrel{\circ}{L}_{t}^{j} \stackrel{\circ}{L}_{m k} \Theta^{t} \Theta^{m} \Theta^{k}-\Theta^{j} \stackrel{\circ}{L}_{m}^{\ell} \stackrel{\circ}{L}_{\ell k} \Theta^{m} \Theta^{k}\right\} .
$$

This is the proposed relation (B.9) for the surface measure.

\section{REFERENCES}

[1] R.A. Adams: Sobolev Spaces. Academic Press, New York 1975.

[2] S.V. Borodachov, D.P. Hardin, E.B. Saff: Asymptotics for discrete weighted minimal Riesz energy problems on rectifiable sets. Transactions American Math. Soc. 360 (2008) 1559-1580.

[3] S.V. Borodachov, D.P. Hardin, E.B. Saff: Low complexity methods for discretizing manifolds via Riesz energy minimization. Found. Comput. Math. 14 (2014) 1173-1208.

[4] J.S. Brauchart, D.P. Hardin, E.B. Saff: Riesz energy and sets of revolution in $\mathbb{R}^{3}$. Contemporary Math. Functional Analysis and Complex Analysis 481 AMS (2009) 47-57.

[5] J. Deny: Les potentiels d'énergy finie, Acta Math. 82 (1950) 107-183.

[6] P.D. Dragnev, E.B. Saff: Riesz spherical potentials with external fields and minimal energy points separation. Potential Analysis 26 (2007) 139-162.

[7] J. Hadamard: Le problème de Cauchy et les équations aux dérivées partielles linéaires hyperboliques. Hermann, Paris 1932.

[8] H. Harbrecht, W.L. Wendland, N. Zorii: On Riesz minimal energy problems. J. Math. Anal. Appl. 393 (2012) 397-412. 
[9] H. Harbrecht, W.L. Wendland, N. Zorii: Riesz minimal energy problems on $C^{k-1,1}$-manifolds. Math. Nachr. 287 (2014) 48-69.

[10] D.P. Hardin, E.B. Saff: Discretizing manifolds via minimum energy points. Notices AMS 51 (2004) 1186-1194.

[11] D.P. Hardin, E.B. Saff: Minimal Riesz energy point configurations for rectifiable $d$-dimensional manifolds. Advances in Mathematics 193 (2005) 174-204.

[12] G.C. Hsiao, W.L. Wendland: Boundary Integral Equations. Springer, Berlin 2008.

[13] W. Kühnel: Differential Geometry: Curves-Surfaces-Manifolds, 2nd Edition. American Mathematical Society 2006.

[14] A.B.J. Kuijlaars and E.B. Saff: Asymptotics for minimal discrete energy on the sphere Trans. Amer. Math. 350 (1998) 523-538.

[15] N.S. Landkof: Foundations of Modern Potential Theory. Springer, Berlin 1992.

[16] E. Martensen: Potentialtheorie. B.G. Teubner, Stuttgart 1968.

[17] S.G. Mikhlin, S. Prössdorf: Singular Integral Operators. Springer-Verlag Berlin 1986.

[18] G. Of, W.L. Wendland, N. Zorii: On the numerical solution of minimal energy problems. Complex Variables and Elliptic Equations 55 (2010) 991-1012.

[19] M. Ohtsuka: On potentials in locally compact spaces, J. Sci. Hiroshima Univ. Ser. A1 25 (1961) 135-352.

[20] M.E. Taylor: Pseudodifferential Operators. Princeton University Press, Princeton 1981.

[21] W.L. Wendland, O. Steinbach: Analysis. B.G. Teubner, Wiesbaden 2005.

Helmut Harbrecht, Departement Mathematik und Informatik, Universität Basel, Spiegelgasse 1 , 4051 BASEL, SWITZERLAND

Wolfgang L. Wendland, Institut für Angewandte Analysis und Numerische Simulation, Universität Stuttgart, Pfaffenwaldring 57, 70569 Stuttgart, Germany

Natalia Zorit, Institute of Mathematics, National Academy of Sciences of Ukraine, Tereshchenkivska 3, 01601, KYIV-4, UKRAINe 